\title{
PERTURBATIONS OF PLANAR ALGEBRAS
}

\section{Paramita Das, Shamindra Kumar Ghosh and Ved Prakash Gupta}

\begin{abstract}
We analyze the effect of pivotal structures (on a 2-category) on the planar algebra associated to a 1-cell as in Gho and come up with the notion of perturbations of planar algebras by weights (a concept that appeared earlier in Michael Burns' thesis (Bur); we establish a one-to-one correspondence between weights and pivotal structures. Using the construction of [Gho, to each bifinite bimodule over $I I_{1}$-factors, we associate a bimodule planar algebra in such a way that extremality of the bimodule corresponds to sphericality of the planar algebra. As a consequence of this, we reproduce an extension of Jones' theorem (Jon2]) (of associating 'subfactor planar algebras' to extremal subfactors). Conversely, given a bimodule planar algebra, we construct a bifinite bimodule whose associated bimodule planar algebra is the one which we start with, using perturbations and Jones-Walker-Shlyakhtenko-Kodiyalam-Sunder method of reconstructing an extremal subfactor from a subfactor planar algebra. The perturbation technique helps us to construct an example of a family of non-spherical planar algebras starting from a particular spherical one; we also show that this family is associated to a known family of subfactors constructed by Jones.
\end{abstract}

\section{INTRODUCTION}

In the pioneering and celebrated work [Jon1] of Jones, the theory of subfactors saw a new opening with, among other ideas and results, the introduction of the concepts of index and the tower of basic construction for subfactors, which, over the years, had various applications in the understanding of $I I_{1}$-factors, knot theory, quantum groups, TQFTs and other fields. There have been a lot of pathbreaking works in this theory since its initiation - see, for instance, [Jon1, PP1, PP2, Pop1, Pop3, Pop4.

Further, an important aspect of this paper of Jones was the evolution of an invariant called the standard invariant of the subfactor, which basically consists of a grid of finite dimensional $C^{*}$-algebras with some rich structure. It has turned out that, among other invariants, the standard invariant of a finite index subfactor is its most important invariant. An instance to justify this claim is that, for certain 'good' family of subfactors (namely, the amenable ones, see Pop3), their standard invariants turn out to be complete invariants. As such, it was motivating enough for people to work on obtaining a better understanding of this invariant. Sorin Popa (in Pop4) gave an algebraic axiomatization of the standard invariant as a grid of finite dimensional $C^{*}$-algebras, which he called a standard $\lambda$-lattice. Conversely, given such a $\lambda$-lattice, he constructed an extremal subfactor whose standard invariant is the $\lambda$-lattice which he started with. Ocneanu also came up with a 'group-like' structure on the standard invariant and called them paragroups. Subsequently, Vaughan Jones (in [Jon2]) developed a very effective pictorial reformulation of the standard invariant which he called planar algebra, and associated a planar algebra satisfying certain natural conditions (referred as subfactor planar algebra) to any extremal subfactor. In the converse direction, starting from a subfactor planar algebra Jones reconstructed a subfactor whose associated planar algebra is isomorphic to the given one, using Popa's characterization of $\lambda$-lattices. Later, in Pop5, Popa extended his correspondence to generalized $\lambda$-lattices on one hand and finite index subfactors (not necessarily extremal) on the other. In 2003, Michael Burns in his thesis (see $[\mathrm{Bur}$ ), established a similar correspondence replacing generalized $\lambda$-lattices with planar algebras satisfying appropriate conditions (without any assumption of sphericality). Very recently, reconstruction of extremal subfactor from a subfactor planar algebra has also been performed using random matrix and free probability techniques in GJS followed by simpler treatments using planar algebra machinery in JSW and KS2. Planar algebra techniques have recently found applications in developing new methods of constructing certain class of subfactors as well.

Key words and phrases. Planar Algebras, Bicategories, Subfactors.

Das and Ghosh were supported by K. U. Leuven BOF Research Grant OT/08/032 and Gupta was supported by ERC Starting Grant VNALG-200749. 
We now present a brief outline of the motivation and the results that brought this article into existence.

(I) Our motivation stemmed solely from the investigation of the following:

(a) A construction of a planar algebra starting from a 1-cell in a pivotal 2-category was given by the second named author in his thesis (see Gho]). This construction was purely algebraic, with the description of the action of tangles being given in terms of graphical calculus of morphisms, analogous to the ones used in Kas. However, the actual manifestation of the pivotal structure in the planar picture remained unclear and required further analysis.

(b) In the operator algebra context, a nice prototype is the 2-category of bifinite bimodules over $I I_{1}$-factors. So, one would like to investigate the planar algebras obtained using the method in Gho from a bifinite bimodule ${ }_{A} \mathcal{H}_{B}$ where $A$ and $B$ are $I I_{1}$ factors.

(c) Another question in this context is whether the Jones' planar algebra associated to an extremal finite index subfactor $N \subset M$, is isomorphic to the planar algebra coming from the bimodule ${ }_{N} L^{2}(M)_{M}$.

In this paper, we make an attempt to answer these and other natural questions, the answers of which we list below:

(II) Summary of the main results:

(a) We answer the question (I)(c), that is, we show that one does not always get back Jones' subfactor planar algebra from the construction in Gho unless the right pivotal structure is chosen.

(b) In order to find out the exact dependence of the planar algebra from the construction in Gho on the pivotal structure, we come up with the concept of weights of a planar algebra and perturbations of planar algebras by weights; later, we realized that such objects also appeared in Michael Burns' thesis to prove Jones' theorem for non-extremal subfactors. Finally, we establish a one-to-one correspondence between weights on a planar algebra and pivotal structures on the associated 2-category

(c) To each bifinite bimodule over $I I_{1}$-factors, using the construction in [Gho, we associate a bimodule planar algebra in such a way that extremality of the bimodule corresponds to sphericality of the planar algebra. Moreover, this also shows that bimodules with different left and right dimensions, gives the right platform to investigate planar algebras with different modulii coming from shaded and unshaded loops.

(d) Conversely, given a bimodule planar algebra, we construct a bifinite bimodule whose associated bimodule planar algebra is the one that we start with, using perturbations and Jones-Walker-ShlyakhtenkoKodiyalam-Sunder method of reconstructing an extremal subfactor from a subfactor planar algebra.

(e) We give explicit construction of examples of non-spherical planar algebras; more precisely we show that the perturbation class of the diagonal planar algebra with respect to the free group $\mathbb{F}_{2}$, generated by two free generators and trivial cocycle contains a continuum of non-spherical unimodular bimodule planar algebras with index greater than 4; we also prove that this family is associated to a known family of subfactors constructed by Jones.

(III) Some nice consequences:

(a) As a consequence of (II)(c), we reproduce an extension of Jones' theorem (of associating 'subfactor planar algebras' to extremal subfactors). This was proved earlier by Michael Burns in his thesis [Bur; the reconstruction of a non-extremal subfactor had appeared in Pop5.

(b) We show that the perturbation class of a bimodule planar algebra contains a unique spherical unimodular bimodule planar algebra which can also be characterized by the minimality of its index. Subfactor version of such results had appeared in the works of Hiai and Popa.

All results in this article are derived using standard facts on bimodules, subfactors and planar algebras, which can be found, for instance, in [Bis, Jon2, JS, PP1, PP2, Pop1, Pop3, Pop4.

We now briefly describe the organization of this paper. Section 2 serves as a quick recollection of various definitions, standard facts and basic aspects of planar algebras, pivotal bicategories and the bicategory of bifinite bimodules.

In Section 3, we define weight of a planar algebra $P$ and perturbation of $P$ by the weight. A planar algebra with modulus $\left(\delta_{-}, \delta_{+}\right)$can be normalized, that is, perturbed with an appropriate scalar weight to get a unimodular planar algebra (that is, having $\delta_{-}=\delta_{+}$) although the index (:= the product of the $\delta^{\prime}$ 's) 
remains unchanged. If the actions of the 0 -tangles in the normalization are invariant under spherical isotopy, then the planar algebra is called spherical; this is a slight modification of Jones' definition of sphericality in order to accommodate non-unimodular planar algebras. At the very end of this section, we make few immediate observations involving perturbations, $*$-structures and positivity in planar algebras.

In the first part of Section 4, we associate a strict 2-category to a planar algebra and show that weights of the planar algebra are in one-to-one correspondence with pivotal structures on the associated 2-category. Conversely, if we start with a bicategory with two pivotal structures, then the planar algebras obtained from any 1-cell using Gho method, are perturbations of each other. This section ends with a quick recollection (from [Gho) of the method of associating a planar algebra to an 1-cell in a strict 2-category.

Section 5 is an omnibus section and is the crux of this paper. In this section, we first formalize what we mean by a bimodule planar algebra; then, following the above-mentioned method of Gho, we associate a bimodule planar algebra to each bifinite bimodule such that the extremality of the bimodule exactly corresponds to the sphericality of its associated planar algebra. This, in turn, provides an extension to Jones' theorem [Jon2, Theorem 4.2.1], that is, we associate a unimodular bimodule planar algebra to an arbitrary finite index subfactor. Such extension was also obtained by Burns; however, our techniques are completely independent and rely on simple graphical calculus of morphisms in the pivotal 2 category. In the converse direction, given any bimodule planar algebra, we obtain a bifinite bimodule whose associated bimodule planar algebra is isomorphic to the one that we started with, through the application of perturbations and following the strategy of [JSW, KS2].

In the first part of Section 6 , we show that the perturbation class of every bimodule planar algebra contains a unique spherical unimodular bimodule planar algebra which can also be characterized by the property of having the minimal index in the perturbation class. Minimizing indices of conditional expectations onto a subfactor already appeared in the literature in the work of Hiai (in [Hia]) and then Popa (in [Pop3]); we are now able to connect this circle of ideas with our notion of perturbation of planar algebra. In the second part, we construct concrete examples of nonspherical planar algebras purely algebraically; more precisely we show that the perturbation class of the diagonal planar algebra with respect to the free group $\mathbb{F}_{2}$, generated by two free generators and trivial cocycle contains a continuum of non-spherical unimodular bimodule planar algebras with index greater than 4 . As suggested by Jones, we prove that these planar algebras are isomorphic to the ones associated to the (non-extremal) subfactors that he constructed in [Jon1] in order to prove that every index greater than 4 is realized.

In the final section, we discuss some questions pertaining to perturbations and weights of a planar algebra.

\section{Preliminaries}

This section is mainly a recollection of various definitions, standard facts and setting up of notations which will be used in the subsequent sections.

2.1. Planar algebras. Since its inception in [Jon2, the formalism of planar algebras has undergone gradual modifications - see, for instance, Jon2, Jon3, KS1, Gho. The starting ingredient for defining a planar algebra is the operad of tangles. A tangular diagram $T$ consists of a subset $D_{0}$ (referred as the external disc) of $\mathbb{R}^{2}$, homeomorphic to the unit disc along with: (a) finitely many (possibly none) non-intersecting subsets $D_{1}, \ldots, D_{b}$ (referred as internal discs) in the interior of $D_{0}$, each of which is also homeomorphic to the unit disc, (b) the boundary of each disc (internal or external) having even number of marked points numbered clockwise, (c) non-intersecting paths (called strings) in $D_{0} \backslash\left[\bigcup_{i=1}^{b} \operatorname{Int}\left(D_{i}\right)\right]$, which are either loops or meet the boundaries of the discs exactly at two distinct marked points in such a way that every marked point is an endpoint of a string and (d) a checker-board shading on the connected components of $\operatorname{Int}\left(D_{0}\right) \backslash$ $\left[\left(\bigcup_{i=1}^{b} D_{i}\right) \cup\{\right.$ strings $\left.\}\right]$. We will usually indicate the checker-board shading and the numbering of the marked points simply by putting a - (resp., +) sign in the shaded (resp., unshaded) connected component near the boundary segment between the last and first marked points. Such a sign along with half the number of marked points on a disc is called its color. A tangle is the class of a tangular diagram under the equivalence of planar isotopy (preserving the shading and the distinguished boundary components). If a tangle $T$ has $b>0$ (resp., no) internal disc(s) and the color of the disc $D_{i}$ is $\varepsilon_{i} k_{i}$ for $0 \leq i \leq b$, then the tangle is usually 
expressed as $T:\left(\varepsilon_{1} k_{1}, \ldots, \varepsilon_{b} k_{b}\right) \rightarrow \varepsilon_{0} k_{0}$ (resp., $T: \emptyset \rightarrow \varepsilon_{0} k_{0}$ ). See Figure 2.1 for illustrations. Before we proceed further, we fix some notations.

(1) We will consider the natural binary operation on $\{-,+\}$ given by $++:=+,+-:=-,-+:=-$ and $--:=+$.

(2) In a tangle, we will replace (isotopically) parallel strings by a single strand labelled by the number of strings, and an internal disc with color $\varepsilon k$ will be replaced by a bold dot with the sign $\varepsilon$ placed at the angle corresponding to the distinguished boundary component of the disc. For example, है will be replaced by $\varepsilon q_{4}^{2}$.

(3) We will denote the set of all possible colors of discs in tangles by $C o l:=\left\{\varepsilon k: \varepsilon \in\{+,-\}, k \in \mathbb{N}_{0}\right\}$ where $\mathbb{N}_{0}:=\mathbb{N} \cup\{0\}$.

There is a natural notion of composition of tangles under which the tangles listed in Figure 2.1 generate the whole operad of tangles. (See [KS1.)

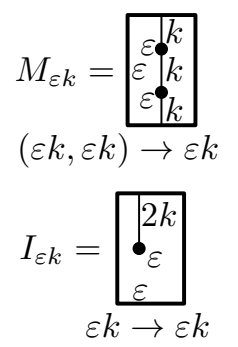

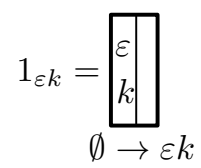

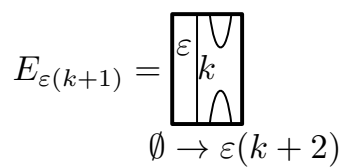

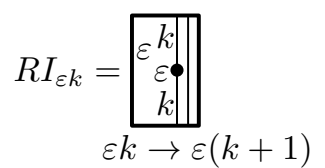

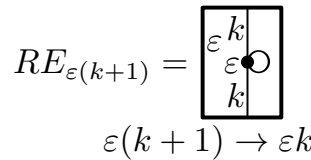

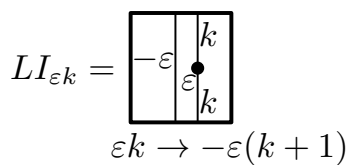

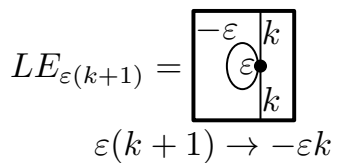

Figure 2.1. Generating tangles.

A planar algebra $P$ is a 'representation' of the operad of tangles, that is, it consists of complex vector spaces $\left\{P_{\varepsilon k}: \varepsilon k \in C o l\right\}$ and for every tangle $T:\left(\varepsilon_{1} k_{1}, \varepsilon_{2} k_{2}, \ldots, \varepsilon_{b} k_{b}\right) \rightarrow \varepsilon_{0} k_{0}$ (resp., $\left.T: \emptyset \rightarrow \varepsilon_{0} k_{0}\right)$, there exists an action of $T$ given by a multi-linear map $P_{T}: \times_{i=1}^{b} P_{\varepsilon_{i} k_{i}} \rightarrow P_{\varepsilon_{0} k_{0}}$ (resp., a vector $P_{T} \in P_{\varepsilon_{0} k_{0}}$ ) such that the action preserves (i) composition and (ii) identity (that is, $P_{I_{\varepsilon k}}=i d_{P_{\varepsilon k}}$ ). Note that $\left\{P_{\varepsilon k}\right\}_{k \in \mathbb{N}_{0}}$ has a unital filtered algebra structure with multiplication, unit and inclusion given by the actions of $M_{\varepsilon k}, 1_{\varepsilon k}$ and $R I_{\varepsilon k}$, respectively.

Definition 2.1. A planar algebra $P$ is said to

(1) be connected (resp., finite dimensional) if $\operatorname{dim}\left(P_{ \pm 0}\right)=1$ (resp., $\operatorname{dim}\left(P_{\varepsilon k}\right)<\infty$ for all $\varepsilon k \in$ Col).

(2) have modulus $\left(\delta_{-}, \delta_{+}\right) \in \mathbb{C}^{2}$ if $P \underset{\Xi}{\Xi}=\delta_{ \pm} P_{1_{ \pm 0}}=\delta_{ \pm} 1_{P_{ \pm 0}}$ and in this case, the scalar $\delta_{+} \delta_{-}$is called the index of $P$.

(3) be unimodular if it has modulus $\left(\delta_{-}, \delta_{+}\right)$such that $\delta_{-}=\delta_{+}$.

(4) be a *-planar algebra if there exists a conjugate-linear involutions $*: P_{\varepsilon k} \rightarrow P_{\varepsilon k}$ for all $\varepsilon k \in C o l$ satisfying the $*$-condition:

$$
P_{T}\left(x_{1}, \ldots, x_{b}\right)^{*}=P_{T^{*}}\left(x_{1}^{*}, \ldots, x_{b}^{*}\right) \quad\left(\text { resp. },\left(P_{T}\right)^{*}=P_{T^{*}}\right)
$$

for each tangle $T:\left(\varepsilon_{1} k_{1} \times \cdots \times \varepsilon_{b} k_{b}\right) \rightarrow \varepsilon_{0} k_{0}$ (resp., $\left.T: \emptyset \rightarrow \varepsilon_{0} k_{0}\right)$ and $x_{i} \in P_{\varepsilon_{i} k_{i}}, 1 \leq i \leq b$, where the adjoint $T^{*}$ of a tangle $T$ is obtained by reflecting it about a horizontal line keeping the shading and distinguished boundary components intact.

(5) be $C^{*}$-planar algebra if each $P_{\varepsilon k}$ is a $C^{*}$-algebra such that its multiplication is the same as that induced by $M_{\varepsilon k}$ and $P$ forms a $*$-planar algebra with respect to the $*$.

(6) be irreducible if $\operatorname{dim}\left(P_{+1}\right)=1$ (equivalently, $\operatorname{dim}\left(P_{-1}\right)=1$ )

Note that a connected planar algebra $P$ has modulus, and two canonical picture traces $P_{T R_{\varepsilon k}^{r}}: P_{\varepsilon k} \rightarrow P_{\varepsilon 0} \cong \mathbb{C}$ and $P_{T R_{\varepsilon k}^{l}}: P_{\varepsilon k} \rightarrow P_{(-)^{k} \varepsilon 0} \cong \mathbb{C}$ induced by the trace tangles $T R_{\varepsilon k}^{r}:=\begin{aligned} & \varepsilon \\ & \varepsilon \\ & \varepsilon\end{aligned}$ and $T R_{\varepsilon k}^{l}:=\begin{aligned} & (-)^{k} \varepsilon \\ & k(\varepsilon)\end{aligned}$. A connected $C^{*}$-planar algebra $P$ is said to be positive if the canonical picture traces are positive definite. 
We now recall the definition of the $n$-th dual of a planar algebra $P$ with modulus $\left(\delta_{-}, \delta_{+}\right)$, denoted by $\lambda_{n}(P)$. For a tangle $T$, let $\lambda_{n}(T)$ be the tangle obtained from $T$ by attaching $n$ parallel string on the disc $D$ (internal or external) enclosing closely the sign $\varepsilon_{D}$ near the distinguished boundary component of the disc. Vectors spaces: For all colors $\varepsilon k, \lambda_{n}(P)_{\varepsilon k}:=\operatorname{Range}\left(P_{\lambda_{n}\left(I_{\varepsilon k}\right)}\right)$.

Action of tangles: For all tangle $T, \lambda_{n}(P)_{T}:=\left[\prod_{D \in\{\text { internal disc(s) of } T\}}\left(\prod_{l=1}^{n} \delta_{(-)^{l} \varepsilon_{D}}\right)^{-1}\right] P_{\lambda_{n}(T)}$.

Note that $\lambda_{0}(P)=P, \lambda_{n}\left(\lambda_{m}(P)\right)=\lambda_{m+n}(P)$ and $\lambda_{n}(P)$ has modulus $\left(\delta_{(-)^{n+1}}, \delta_{(-)^{n}}\right)$ for all $m, n \in \mathbb{N}_{0}$.

For any mathematical category, studying the morphisms in that category is very crucial. A morphism $\varphi: P \rightarrow Q$ for two planar algebras $P$ and $Q$, is a collection of linear maps $\varphi_{\varepsilon k}: P_{\varepsilon k} \rightarrow Q_{\varepsilon k}, \varepsilon k \in C o l$ which are equivariant with the action of tangles (that is, $\varphi \circ P_{T}=Q_{T} \circ \varphi$ or $\varphi \circ P_{T}=Q_{T}$ according as $T$ has at least one internal disc or none). Given such a double sequence of linear maps, it is not always necessary to verify its equivariance with every tangle. For instance, it suffices to do the same only for the actions of a generating set of tangles mentioned in Figure 2.1

Remark 2.2. Let $P$ and $Q$ be connected planar algebras with non-zero moduli. Then, a linear map $\varphi: P \rightarrow Q$ is a planar algebra morphism if it is equivariant with the actions of any of the following sets of tangles:

(1) $\cup_{k \in \mathbb{N}_{0}}\left\{M_{ \pm k}, R I_{ \pm k}, L I_{ \pm k}, E_{ \pm 1}\right\}$

(2) $\underset{k \in \mathbb{N}_{0}}{\cup}\left\{M_{+k}, R I_{+k}, L I_{-k}, E_{+(k+1)}, L E_{+(k+1)}\right\}$

Sometimes, in order to obtain morphisms between planar algebras, it is even enough to obtain a morphism on the 'positive parts'. More precisely, we have the following lemma.

Lemma 2.3. Let $P$ and $Q$ be planar algebras having the same pair of non-zero moduli, say $\left(\delta_{-}, \delta_{+}\right)$, and suppose there exist linear maps $\varphi_{+k}: P_{+k} \rightarrow Q_{+k}, k \geq 0$, equivariant with the action of tangles with discs only of positive colors. Then there exists a unique planar algebra morphism $\tilde{\varphi}: P \stackrel{\sim}{\rightarrow} Q$ such that $\varphi_{+k}=\tilde{\varphi}_{+k}$ for all $k \geq 0$. Moreover, if $\varphi$ is an isomorphism, so is $\tilde{\varphi}$.

Proof: Consider $\tilde{\varphi}: P \rightarrow Q$ given by $\tilde{\varphi}_{+k}=\varphi_{+k}$ and

$$
P_{-k} \ni x \stackrel{\tilde{\varphi}_{-k}}{\longmapsto} \delta_{-}^{-1} Q_{L E_{+(k+1)}} \circ \varphi_{+(k+1)} \circ P_{L I_{-k}}(x) \in Q_{-k}
$$

for all $k \geq 0$. It is straight forward to check that $\tilde{\varphi}$ is equivariant with the action of tangles of all types (not necessarily having positive colors for all discs). The uniqueness follows from the definition.

For the second part, since $\varphi$ is equivariant with the action of $E_{+(k+1)}^{\prime}:=L I_{-k} \circ L E_{+(k+1)}$, we have $\varphi_{+(k+1)}\left(\operatorname{Ran} P_{E_{+(k+1)}^{\prime}}\right) \subseteq \operatorname{Ran} Q_{E_{+(k+1)}^{\prime}}$, with equality if $\varphi$ is surjective; also, $\varphi_{-k}$ is injective if so is $\varphi$ because $Q_{L E_{+(k+1)}}$ restricted to $\operatorname{Ran} Q_{E_{+(k+1)}^{\prime}}$ is injective. Thus, $\tilde{\varphi}$ is an isomorphism if so is $\varphi$.

Let $P$ be a planar algebra. Then, by a $P$-labelled (resp., semi-labelled) tangle, we mean a tangle whose all (resp., some) internal discs are labelled by elements of $P$ such that an internal disc of color $\varepsilon k$ is labelled by an element of $P_{\varepsilon k}$. For simplicity, we will replace a $P$-labelled internal disc by a bold dot as before with the label being placed at the angle corresponding to the distinguished boundary component of the disc.

2.2. Bicategories. In this subsection, for the sake of completeness, we recall the notion of bicategories and structures of rigidity and pivotality on them which will be used later. Most of the material in this subsection can be found in any standard textbook on bicategories.

Definition 2.4. A bicategory $\mathcal{B}$ consists of:

- a class $\mathcal{B}_{0}$ whose elements are called 0 -cells;

- for $\alpha, \beta \in \mathcal{B}_{0}$, a category $\mathcal{B}(\alpha, \beta)$ whose object $X$ shall be called 1 -cells and denoted by $\alpha \stackrel{X}{\rightarrow} \beta$, and whose morphism $X_{1} \stackrel{f}{\rightarrow} X_{2}$ from $\alpha \stackrel{X_{1}}{\rightarrow} \beta$ to $\alpha \stackrel{X_{2}}{\rightarrow} \beta$ shall be called 2 -cells;

- for $\alpha, \beta, \gamma \in \mathcal{B}_{0}$, there exists a functor $\otimes: \mathcal{B}(\beta, \gamma) \times \mathcal{B}(\alpha, \beta) \rightarrow \mathcal{B}(\alpha, \gamma)$;

- Associativity constraint: For each triple $\alpha \stackrel{X}{\rightarrow} \beta, \beta \stackrel{Y}{\rightarrow} \gamma, \gamma \stackrel{Z}{\rightarrow} \delta$ of 1-cells, there exists an isomorphism $(Z \otimes Y) \otimes X \stackrel{\alpha_{Z, Y} X}{\longrightarrow} Z \otimes(Y \otimes X)$ in $\operatorname{Mor}(\mathcal{B}(\alpha, \delta))$

- Identity object: for each 0 -cell $\alpha$, there exists a 1 -cell $\alpha \stackrel{1_{\alpha}}{\rightarrow} \alpha$ (called the identity on $\alpha$ ); 
- Unit Constraint: for each 1 -cell $\alpha \stackrel{X}{\rightarrow} \beta$, there exist isomorphisms $1_{\beta} \otimes X \stackrel{\lambda_{x}}{\rightarrow} X$ and $X \otimes 1_{\alpha} \stackrel{\rho_{x}}{\rightarrow} X$ in $\operatorname{Mor}(\mathcal{B}(\alpha, \beta))$

such that $\alpha_{Z, Y, X}, \lambda_{X}$ and $\rho_{X}$ are natural in $Z, Y$ and $X$, and satisfy the pentagon and the triangle axioms (which are exactly similar to the ones in the definition of a tensor category).

On a bicategory $\mathcal{B}$, one can perform the operation op (resp., $c o$ ) and obtain a new bicategory $\mathcal{B}^{o p}$ (resp., $\mathcal{B}^{c o}$ ) by setting (i) $\mathcal{B}_{0}^{o p}=\mathcal{B}_{0}=\mathcal{B}_{0}^{c o}$, (ii) $\mathcal{B}^{o p}(\beta, \alpha)=\mathcal{B}(\alpha, \beta)=\left(\mathcal{B}^{c o}(\alpha, \beta)\right)^{o p}$ as categories (where op of a category is basically reversing the directions of the morphisms).

A bicategory will be called a strict 2-category if the associativity and the unit constraints are identities. A $\mathbb{C}$-linear bicategory $\mathcal{B}$ is a bicategory such that $\mathcal{B}(\alpha, \beta)$ is a $\mathbb{C}$-linear category for every $\alpha, \beta \in \mathcal{B}_{0}$ and the functor $\otimes$ is additive.

Definition 2.5. Let $\mathcal{B}, \mathcal{B}^{\prime}$ be bicategories. A weak functor $F=(F, \varphi): \mathcal{B} \rightarrow \mathcal{B}^{\prime}$ consists of:

- a function $F: \mathcal{B}_{0} \rightarrow \mathcal{B}_{0}^{\prime}$,

- for all $\alpha, \beta \in \mathcal{B}_{0}$, there exists a functor $F^{\alpha, \beta}: \mathcal{B}(\alpha, \beta) \rightarrow \mathcal{B}^{\prime}(F(\alpha), F(\beta))$ written simply as $F$,

- for all $\alpha, \beta, \gamma \in \mathcal{B}_{0}$, there exists a natural isomorphism $\varphi^{\alpha, \beta, \gamma}: \otimes^{\prime} \circ\left(F^{\beta, \gamma} \times F^{\alpha, \beta}\right) \rightarrow F^{\alpha, \gamma} \circ \otimes$ written simply as $\varphi$ (where $\otimes$ and $\otimes^{\prime}$ are the tensor functors of $\mathcal{B}$ and $\mathcal{B}^{\prime}$ respectively),

- for all $\alpha \in \mathcal{B}_{0}$, there exists an invertible (with respect to composition) 2-cell $\varphi_{\alpha}: 1_{F(\alpha)} \rightarrow F\left(1_{\alpha}\right)$,

satisfying commutativity of certain diagrams (consisting of 2-cells) which are analogous to the hexagonal and rectangular diagrams appearing in the definition of a tensor functor.

Definition 2.6. Let $F=(F, \varphi), G=(G, \psi): \mathcal{B} \rightarrow \mathcal{B}^{\prime}$ be weak functors. A weak transformation $\sigma: F \rightarrow G$ consists of:

- for all $\alpha \in \mathcal{B}_{0}$, there exists a 1-cell $\sigma_{\alpha} \in$ ob $\left(\mathcal{B}^{\prime}(F(\alpha), G(\alpha))\right)$,

- for all $\alpha, \beta \in \mathcal{B}_{0}$, there exists a natural transformation $\sigma^{\alpha, \beta}:\left(\sigma_{\beta} \otimes^{\prime} F^{\alpha, \beta}\right) \rightarrow G^{\alpha, \beta} \otimes^{\prime} \sigma_{\alpha}$ written simply as $\sigma$ (where $\left(\sigma_{\beta} \otimes^{\prime} F^{\alpha, \beta}\right), G^{\alpha, \beta} \otimes^{\prime} \sigma_{\alpha}: \mathcal{B}(\alpha, \beta) \rightarrow \mathcal{B}(F(\alpha), G(\beta))$ are functors defined in the obvious way), satisfying the following:

for all $X \in o b(\mathcal{B}(\beta, \gamma)), Y \in o b(\mathcal{B}(\alpha, \beta))$ where $\alpha, \beta, \gamma \in \mathcal{B}_{0}$, the following two diagrams commute:

$$
\begin{aligned}
& \sigma_{\gamma} \otimes^{\prime} F(X) \otimes^{\prime} F(Y) \stackrel{\sigma_{X} \otimes^{\prime} i d_{F(Y)}}{\longrightarrow} G(X) \otimes^{\prime} \sigma_{\beta} \otimes^{\prime} F(Y) \stackrel{i d_{G(X)} \otimes^{\prime} \sigma_{Y}}{\longrightarrow} G(X) \otimes^{\prime} G(Y) \otimes^{\prime} \sigma_{\alpha} \\
& i d_{\sigma_{\gamma}} \otimes^{\prime} \varphi_{X, Y} \downarrow \quad \downarrow \psi_{X, Y} \otimes^{\prime} i d_{\sigma_{\alpha}} \\
& \sigma_{\gamma} \otimes^{\prime} F(X \otimes Y) \longrightarrow G(X \otimes Y) \otimes^{\prime} \sigma_{\alpha} \\
& \sigma_{\alpha} \otimes^{\prime} i d_{F(\alpha)} \stackrel{\rho_{\sigma_{\alpha}}^{\prime}}{\longrightarrow} \sigma_{\alpha} \longleftarrow \lambda_{\sigma_{\alpha}}^{\prime} i d_{G(\alpha)} \otimes^{\prime} \sigma_{\alpha} \\
& \sigma_{\alpha} \otimes^{\prime} F\left(1_{\alpha}\right) \longrightarrow G\left(1_{\alpha}\right) \otimes^{\prime} \sigma_{\alpha}
\end{aligned}
$$

where $\lambda^{\prime}$ and $\rho^{\prime}$ are the left and right unit constraints of $\mathcal{B}^{\prime}$ respectively.

When such a weak transformation exists, we say that $F$ and $G$ are weakly isomorphic. We have the following useful Coherence Theorem for bicategories. See [Lei] for a proof.

Theorem 2.7. Every bicategory $\mathcal{B}$ is biequivalent to some strict 2 -category $\mathcal{B}^{\prime}$, i.e., there exist weak functors $F: \mathcal{B} \rightarrow \mathcal{B}^{\prime}$ and $G: \mathcal{B}^{\prime} \rightarrow \mathcal{B}$ such that $i d_{\mathcal{B}}$ (resp., $i d_{\mathcal{B}^{\prime}}$ ) is weakly isomorphic to $G \circ F$ (resp., $F \circ G$ ).

In view of this, time and again we will supress (and will not mention about it) the associativity and unit constraints to give a simpler look to expressions involving these constraints.

Rigid Structure on a bicategory. Let $\alpha \stackrel{X}{\rightarrow} \beta$ be a 1-cell in a bicategory $\mathcal{B}$. A right dual of $X$ is a 1-cell $\beta \stackrel{X^{\#}}{\rightarrow} \alpha$ such that there exist 2-cells $X^{\#} \otimes X \stackrel{e_{X}}{\rightarrow} 1_{\alpha}$ and $1_{\beta} \stackrel{c_{X}}{\rightarrow} X \otimes X^{\#}$ satisfying

$$
\left(i d_{X} \otimes e_{X}\right) \circ\left(c_{X} \otimes i d_{X}\right)=i d_{X} \quad \text { and } \quad\left(e_{X} \otimes i d_{X \#}\right) \circ\left(i d_{X \#} \otimes c_{X}\right)=i d_{X \#} .
$$

A bicategory is said to be (right) rigid if right dual exists for every 1-cell. Further, in a rigid bicategory $\mathcal{B}$, one can consider right dual as an invertible weak functor $\#=(\#, s): \mathcal{B} \rightarrow \mathcal{B}^{o p c o}$ in the following way: 
- for each 1-cell $X$, we fix a triplet $\left(X^{\#}, e_{X}, c_{X}\right)$ so that when $X=1_{\alpha}$ for a 0 -cell $\alpha$, then $X^{\#}=1_{\alpha}$, $e_{X}=\lambda_{1_{\alpha}}\left(=\rho_{1_{\alpha}}\right.$, see [Kas] for a proof $)$ and $c_{X}=\lambda_{1_{\alpha}}^{-1}=\rho_{1_{\alpha}}^{-1}$;

- \# induces identity map on $\mathcal{B}_{0}$;

- for each pair of 0-cells $\alpha$ and $\beta$, define the contravariant functor $\#: \mathcal{B}(\alpha, \beta) \rightarrow \mathcal{B}(\beta, \alpha)$ as follows: for each $X, Y \in o b(\mathcal{B}(\alpha, \beta))$ and 2-cell $f: X \rightarrow Y$, set $\#(X)=X^{\#}$ and $\#(f)$, denoted by $f^{\#}$, be given by the following composition

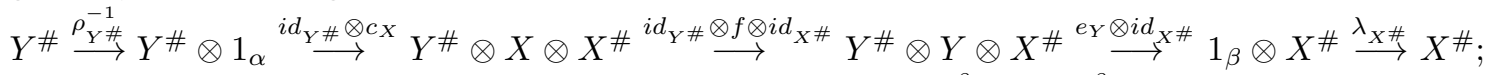

- for all $\alpha, \beta, \gamma \in \mathcal{B}_{0}$, the natural isomorphism $s: \otimes \circ($ flip $) \circ\left(\#^{\beta, \gamma} \times \#^{\alpha, \beta}\right) \rightarrow \#^{\alpha, \gamma} \circ \otimes$ is defined by: for $X \in o b(\mathcal{B}(\alpha, \beta)), Y \in o b(\mathcal{B}(\beta, \gamma))$, the invertible 2-cell $s_{X, Y}$ is given by the composition

$$
\begin{aligned}
& X^{\#} \otimes Y^{\#}{ }^{i d_{\left(X \# \otimes Y^{\#)}\right)} \otimes c_{(Y \otimes X)}} X^{\#} \otimes Y^{\#} \otimes(Y \otimes X) \otimes(Y \otimes X)^{\#} \stackrel{i d_{X \#} \otimes e_{Y} \otimes i d_{X} \otimes i d_{(Y \otimes X) \#}}{\longrightarrow}\left(X^{\#} \otimes X\right) \otimes \\
& (Y \otimes X)^{\#} \stackrel{e_{X} \otimes i d_{(Y \otimes X)}}{\longrightarrow}(Y \otimes X)^{\#} ;
\end{aligned}
$$

- for all $\alpha \in \mathcal{B}_{0}$, the invertible 2-cell $s_{\alpha}: 1_{\alpha} \rightarrow 1_{\alpha}$ is given by identity morphism on $1_{\alpha}$.

Note that the above prescription of the dual functor $(\#, s)$ carries forward almost verbatim to another weak functor $(\tilde{\#}, \tilde{s}): \mathcal{B}^{o p c o} \rightarrow\left(\mathcal{B}^{o p c o}\right)^{o p c o}=\mathcal{B}$. This allows us to consider the composition $(\tilde{\#}, \tilde{s}) \circ(\#, s): \mathcal{B} \rightarrow \mathcal{B}$. This is again a weak functor and we abuse notation to denote it by $(\# \#, t)$ and call it the bi-dual functor.

Definition 2.8. $A$ bicategory $\mathcal{B}$ is said to be pivotal if $\mathcal{B}$ is (right) rigid and there exists a weak transformation $a: i d_{\mathcal{B}} \rightarrow \# \#$ such that $a_{\varepsilon}=1_{\varepsilon}$ for all $\varepsilon \in \mathcal{B}_{0}$.

We now recall some useful standard properties of a pivotal bicategory.

Proposition 2.9. Let $\mathcal{B}$ be a pivotal bicategory with pivotality given by the weak transformation $a: i d_{\mathcal{B}} \rightarrow$ \#\#. Then, for all 1 -cells $\alpha \stackrel{Y}{\rightarrow} \beta$ and $\beta \stackrel{X}{\rightarrow} \gamma$, we have

(1) $a_{X \otimes Y}=t_{X, Y} \circ\left(a_{X} \otimes a_{Y}\right)$ and

(2) $a_{X}^{\#}=a_{X \#}^{-1}$.

Let $\alpha_{i} \stackrel{X_{i}}{\rightarrow} \alpha_{i+1}, 1 \leq i \leq n$ be 1-cells in $\mathcal{B}$. Suppressing associativity, consider the morphism $t_{X_{1}, \ldots, X_{n}}:=$

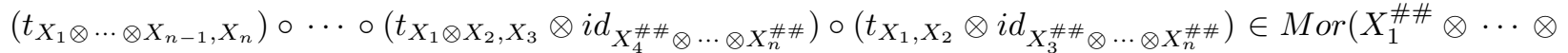

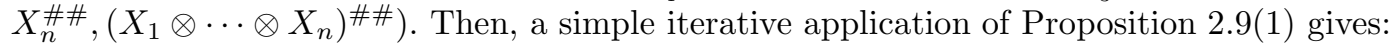

Corollary 2.10. $a_{X_{1} \otimes \cdots \otimes X_{n}}=t_{X_{1}, \ldots, X_{n}} \circ\left(a_{X_{1}} \otimes \cdots \otimes a_{X_{n}}\right)$ for all 1 -cells $\alpha_{i} \stackrel{X_{i}}{\rightarrow} \alpha_{i+1}, 1 \leq i \leq n$.

2.3. Bicategory of bifinite bimodules. In this subsection, for the sake of completeness, we first recall certain standard facts about subfactors and modules over $I I_{1}$-factors (which can be found, for instance, in [Bis, EK, GHJ, Jon1, JS, PP1, PP2, Pop3, Pop4, Sun]). And while doing so, we also illustrate how the collection of all bimodules inherit a canonical structure of a pivotal bicategory. We make a stand-in assumption that all Hilbert spaces are separable and their inner products are linear in second and conjugatelinear in first variable.

The following proposition gives a characterization of the basic construction.

Proposition 2.11. Let $A \subset B \subset C$ be unital inclusions of $I I_{1}$-factors and suppose there is a projection $e \in \mathscr{P}(C)$ satisfying

(1) exe $=E_{A}(x)$ e for all $x \in B$ and

(2) $\mathrm{Be}=\mathrm{Ce}$.

Then the above tower is an instance of basic construction.

Given a finite index subfactor $N \subset M$, a left (resp., right) basis of $M$ over $N$ is a finite subset $B$ of $M$ satisfying any of the following equivalent conditions:

(i) $x=\sum_{b \in B} E_{N}\left(x b^{*}\right) b$ (resp., $x=\sum_{b \in B} b E_{N}\left(b^{*} x\right)$ ) for all $x \in M$,

(ii) $x=\sum_{b \in B} b^{*} E_{N}(b x)$ (resp., $x=\sum_{b \in B} E_{N}(x b) b^{*}$ ) for all $x \in M$,

(iii) $\sum_{b \in B} b^{*} e b=1$ (resp., $\sum_{b \in B} b e b^{*}=1$ ) where $e$ is a Jones projection in a basic construction of $N \subset M$. 
Proof of existence of such basis can be found in [PP1].

Let $A$ and $B$ be $I I_{1}$-factors and ${ }_{A} \mathcal{H}$ (resp., $\mathcal{K}_{B}$ ) be a left $A$-module (resp., right $B$-module) such that $\operatorname{dim}\left({ }_{A} \mathcal{H}\right)<\infty$ (resp., $\operatorname{dim}\left(\mathcal{K}_{B}\right)<\infty$ ), equivalently, $A^{\prime}:={ }_{A} \mathcal{L}(\mathcal{H})$ (resp., $B^{\prime}:=\mathcal{L}_{B}(\mathcal{K})$ ) is a $I I_{1}$-factor. Consider the set of bounded vectors $\left({ }_{A} \mathcal{H}\right)^{o}:=\left\{\xi \in \mathcal{H}\right.$ : there exists $k>0$ s.t. $\|a \xi\|^{2} \leq k \operatorname{tr}_{A}\left(a a^{*}\right)$ for all $a \in$ $A\}$ (resp., $\left(\mathcal{K}_{B}\right)^{o}:=\left\{\eta \in \mathcal{K}\right.$ : there exists $k>0$ s.t. $\|\eta b\|^{2} \leq k \operatorname{tr}_{B}\left(b b^{*}\right)$ for all $\left.b \in B\right\}$ ) which forms a dense subspace of $\mathcal{H}$ (resp., $\mathcal{K}$ ) and is closed under the action of $A^{\prime}$ (resp., $B^{\prime}$ ). Using the Radon-Nikodym derivative with respect to the faithful trace, one can obtain the $A$ - (resp., $B$-) valued inner product ${ }_{A}\langle\cdot, \cdot\rangle$ : $\left({ }_{A} \mathcal{H}\right)^{o} \times\left({ }_{A} \mathcal{H}\right)^{o} \rightarrow A$ (resp., $\langle\cdot, \cdot\rangle_{B}:\left(\mathcal{K}_{B}\right)^{o} \times\left(\mathcal{K}_{B}\right)^{o} \rightarrow B$ ) defined by the equation $\operatorname{tr}_{A}\left(a_{A}\left\langle\xi, \xi^{\prime}\right\rangle\right)=\left\langle\xi, a \xi^{\prime}\right\rangle$ (resp., $\operatorname{tr}_{B}\left(\left\langle\eta, \eta^{\prime}\right\rangle_{B} b\right)=\left\langle\eta, \eta^{\prime} b\right\rangle$ ) for all $\xi, \xi^{\prime} \in\left({ }_{A} \mathcal{H}\right)^{o}, a \in A$ (resp., $\eta, \eta^{\prime} \in\left(\mathcal{K}_{B}\right)^{o}, b \in B$ ). It is easy to check that the inner product has the following properties:

(1) ${ }_{A}\langle\xi, \xi\rangle$ (resp., $\langle\eta, \eta\rangle_{B} \geq 0$ ),

(2) ${ }_{A}\left\langle\xi, \xi^{\prime}\right\rangle^{*}={ }_{A}\left\langle\xi^{\prime}, \xi\right\rangle$ (resp., $\left\langle\eta, \eta^{\prime}\right\rangle_{B}^{*}=\left\langle\eta^{\prime}, \eta\right\rangle_{B}$ ),

(3) ${ }_{A}\left\langle\xi, a \xi^{\prime}\right\rangle=a_{A}\left\langle\xi, \xi^{\prime}\right\rangle$ and ${ }_{A}\left\langle a \xi, \xi^{\prime}\right\rangle={ }_{A}\left\langle\xi, \xi^{\prime}\right\rangle a^{*}$ (resp., $\left\langle\eta, \eta^{\prime} b\right\rangle_{B}=\left\langle\eta, \eta^{\prime}\right\rangle_{B} b$ and $\left\langle\eta b, \eta^{\prime}\right\rangle_{B}=b^{*}\left\langle\eta, \eta^{\prime}\right\rangle_{B}$ ),

(4) ${ }_{A}\left\langle\xi, x \xi^{\prime}\right\rangle={ }_{A}\left\langle x^{*} \xi, \xi^{\prime}\right\rangle$ (resp., $\left.\left\langle\eta, y \eta^{\prime}\right\rangle=\left\langle y^{*} \eta, \eta^{\prime}\right\rangle_{B}\right)$

for all $a \in A, x \in A^{\prime}, \xi, \xi^{\prime} \in\left({ }_{A} \mathcal{H}\right)^{o}$ (resp., $\left.b \in B, y \in B^{\prime}, \eta, \eta^{\prime} \in\left(\mathcal{K}_{B}\right)^{o}\right)$. Also, given a finite index subfactor $C$ of $A$ (resp., $B$ ), one can use a basis for $C \subset A$ (resp., $C \subset B$ ) to obtain $\left({ }_{A} \mathcal{H}\right)^{o}=\left({ }_{C} \mathcal{H}\right)^{o}$ (resp., $\left.\left(\mathcal{K}_{B}\right)^{o}=\left(\mathcal{K}_{C}\right)^{o}\right)$ where the $C$-valued inner product is given by the $A$ - (resp., $B$-) valued inner product composed with the trace preserving conditional expectation onto $C$. Further, there exists a finite subset $\left\{\xi_{i}\right\}_{i}$ (resp., $\left.\left\{\eta_{j}\right\}_{j}\right)$ of $\left({ }_{A} \mathcal{H}\right)^{o}$ (resp., $\left.\left(\mathcal{K}_{B}\right)^{o}\right)$ satisfying $i d_{(A \mathcal{H})^{o}}=\sum_{i}{ }_{A}\left\langle\xi_{i}, \cdot\right\rangle \xi_{i}$ (resp., $\left.i d_{\left(\mathcal{K}_{B}\right)^{o}}=\sum_{j} \eta_{j}\left\langle\eta_{j}, \cdot\right\rangle_{B}\right)$; such a subset is called basis for the module. Such a basis also satisfies the following conditions which are completely straight forward to verify.

Proposition 2.12. (i) $\operatorname{tr}_{A^{\prime}}(x)=\left[\operatorname{dim}\left({ }_{A} \mathcal{H}\right)\right]^{-1} \sum_{i}\left\langle\xi_{i}, x \xi_{i}\right\rangle$ (resp., $\operatorname{tr}_{B^{\prime}}(y)=\left[\operatorname{dim}\left(\mathcal{K}_{B}\right)\right]^{-1} \sum_{j}\left\langle\eta_{j}, \eta_{j} y\right\rangle$ ) for all $x \in A^{\prime}$ (resp., $\left.y \in B^{\prime}\right)$ which implies $\left({ }_{A} \mathcal{H}\right)^{o}=\left({ }_{A^{\prime}} \mathcal{H}\right)^{o}$ (resp.,$\left.\left(\mathcal{K}_{B}\right)^{o}=\left({ }_{B^{\prime}} \mathcal{K}\right)^{o}\right)$,

(ii) $\sum_{i}{ }_{A^{\prime}}\left\langle\xi_{i}, \xi_{i}\right\rangle=\operatorname{dim}\left({ }_{A} \mathcal{H}\right) 1_{A^{\prime}}$ (resp., $\left.\sum_{j} B_{B^{\prime}}\left\langle\eta_{j}, \eta_{j}\right\rangle=\operatorname{dim}\left(\mathcal{K}_{B}\right) 1_{B^{\prime}}\right)$.

The dual Hilbert space or the contragredient $\overline{\mathcal{H}}:=\{\bar{\xi}: \xi \in \mathcal{H}\}$ (resp., $\overline{\mathcal{K}}:=\{\bar{\eta}: \eta \in \mathcal{K}\}$ ) where bar being a conjugate linear unitary, can be equipped with a right $A$ - (resp., left $B$-) module structure given by $\bar{\xi} a=\overline{a^{*} \xi}$ for $a \in A, \xi \in \mathcal{H}$ (resp., $b \bar{\eta}=\overline{\eta b^{*}}$ for $b \in B, \eta \in \mathcal{K}$ ). Note that $(\mathrm{i})\left(\overline{\mathcal{H}}_{A}\right)^{o}=\overline{\left({ }_{A} \mathcal{H}\right)^{o}}\left(\operatorname{resp} .,\left({ }_{B} \overline{\mathcal{K}}\right)^{o}=\overline{\left(\mathcal{K}_{B}\right)^{o}}\right)$, (ii) $\left\langle\bar{\xi}, \overline{\xi^{\prime}}\right\rangle_{A}={ }_{A}\left\langle\xi^{\prime}, \xi\right\rangle$ for $\xi, \xi^{\prime} \in\left({ }_{A} \mathcal{H}\right)^{o}$ (resp., ${ }_{B}\left\langle\bar{\eta}, \overline{\eta^{\prime}}\right\rangle=\left\langle\eta^{\prime}, \eta\right\rangle_{B}$ for $\left.\eta, \eta^{\prime} \in\left(\mathcal{K}_{B}\right)^{o}\right)$, (iii) $\left\{\overline{\xi_{i}}\right\}_{i}$ (resp., $\left\{\overline{\eta_{j}}\right\}_{j}$ ) is a basis for $\overline{\mathcal{H}}_{A}$ (resp., $\left.{ }_{B} \overline{\mathcal{K}}\right)$ and (iv) $\operatorname{dim}\left({ }_{A} \mathcal{H}\right)=\operatorname{dim}\left(\overline{\mathcal{H}}_{A}\right)\left(\right.$ resp., $\left.\operatorname{dim}\left(\mathcal{K}_{B}\right)=\operatorname{dim}\left({ }_{B} \overline{\mathcal{K}}\right)\right)$.

Next, we briefly recall few aspects of bimodules over $I I_{1}$-factors $A$ and $B$. Let $\mathcal{H}$ be an $A$ - $B$-bimodule. If $\operatorname{dim}\left(\mathcal{H}_{B}\right)<\infty$, then the Jones index of the subfactor $A \subset B^{\prime}$ turns out to be $\left[B^{\prime}: A\right]=\operatorname{dim}\left({ }_{A} \mathcal{H}\right) \operatorname{dim}\left(\mathcal{H}_{B}\right)=$ : index $\left({ }_{A} \mathcal{H}_{B}\right)$. A bimodule ${ }_{A} \mathcal{H}_{B}$ is called bifinite if index $\left({ }_{A} \mathcal{H}_{B}\right)<\infty$, and a bifinite bimodule ${ }_{A} \mathcal{H}_{B}$ is called extremal if the canonical traces of the $I I_{1}$-factors $A^{\prime}$ and $B^{\prime}$ coincide on the intertwiner space ${ }_{A} \mathcal{L}_{B}(\mathcal{H}$ ) (which has finite complex dimension due to the finiteness of the index). Note that if index $\left({ }_{A} \mathcal{H}_{B}\right)=1$, then ${ }_{A} \mathcal{H}_{B}$ is an irreducible $A$-B-bimodule. Also, if $\operatorname{index}\left({ }_{A} \mathcal{H}_{B}\right)<\infty$, then $\left({ }_{A} \mathcal{H}\right)^{o}=\left(\mathcal{H}_{B}\right)^{o}$; we will write $\mathcal{H}^{o}$ for this space of bounded vectors.

Given two bifinite bimodules ${ }_{A} \mathcal{H}_{B}$ and ${ }_{B} \mathcal{K}_{C}$, one can consider the tensor product $\mathcal{H} \underset{B}{\otimes} \mathcal{K}$ defined as the completion of the space $\mathcal{H}^{o} \underset{B}{\otimes \mathcal{K}^{o}}:=\frac{\mathcal{H}^{o} \underset{\text { alg }}{\otimes} \mathcal{K}^{o}}{\operatorname{span}\left\{\xi a \otimes \eta-\xi \otimes a \eta \mid a \in A, \xi \in \mathcal{H}^{o}, \eta \in \mathcal{K}^{o}\right\}}$ with respect to the inner product $\left\langle\xi \underset{B}{\otimes} \eta, \xi_{B}^{\prime}{\underset{B}{0}}^{\prime}\right\rangle=\left\langle\xi, \xi^{\prime}{ }_{B}\left\langle\eta, \eta^{\prime}\right\rangle\right\rangle=\left\langle\eta,\left\langle\xi, \xi^{\prime}\right\rangle_{B} \eta^{\prime}\right\rangle$ for $\xi, \xi^{\prime} \in \mathcal{H}^{o}, \eta, \eta^{\prime} \in \mathcal{K}^{o}$, which is equipped with the obvious $A-C$ bimodule structure. The following is a list of very useful properties of this tensor product.

(1) $(\mathcal{H} \underset{B}{\otimes} \mathcal{K})^{o}=\mathcal{H}^{o} \underset{B}{\otimes} \mathcal{K}^{o}$

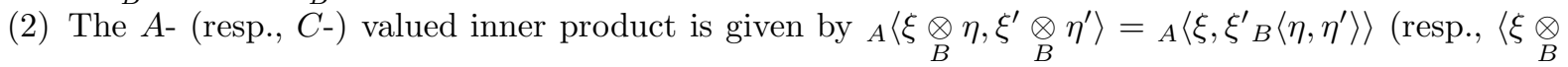
$\left.\left.\eta, \xi_{B}^{\prime} \otimes_{B} \eta^{\prime}\right\rangle_{C}=\left\langle\eta,\left\langle\xi, \xi^{\prime}\right\rangle_{B} \eta^{\prime}\right\rangle_{C}\right)$ for $\xi, \xi^{\prime} \in \mathcal{H}^{o}, \eta, \eta^{\prime} \in \mathcal{K}^{o}$

(3) If $\left\{\xi_{i}\right\}_{i}$ and $\left\{\eta_{j}\right\}_{j}$ are basis for ${ }_{A} \mathcal{H}$ and ${ }_{B} \mathcal{K}$ (resp., $\mathcal{H}_{B}$ and $\mathcal{K}_{C}$ ) respectively, then $\left\{\xi_{i} \underset{B}{\otimes} \eta_{j}\right\}_{i, j}$ forms a basis for ${ }_{A} \mathcal{H} \underset{B}{\otimes} \mathcal{K}$ (resp., $\left.\mathcal{H} \underset{B}{\otimes} \mathcal{K}_{C}\right)$. 
(4) The left dimension, the right dimension and the index of the bifinite bimodules are multiplicative with respect to this tensor product.

(5) The map $\mathcal{L}_{B}(\mathcal{H}) \ni x \mapsto x \underset{B}{\otimes} i d_{\mathcal{K}} \in \mathcal{L}_{C}(\mathcal{H} \underset{B}{\otimes} \mathcal{K})\left(\right.$ resp., ${ }_{B} \mathcal{L}(\mathcal{K}) \ni y \mapsto i d_{\mathcal{H}} \underset{B}{\otimes} y \in{ }_{A} \mathcal{L}(\mathcal{H} \underset{B}{\otimes} \mathcal{K})$ ) is an inclusion of unital $*$-algebras.

Let $\mathcal{H}$ be an $A$ - $B$-bimodule with $\operatorname{dim}\left({ }_{A} \mathcal{H}\right)<\infty$ (resp., $\left.\operatorname{dim}\left(\mathcal{H}_{B}\right)<\infty\right)$ and $\left\{\xi_{i}\right\}_{i}$ (resp., $\left\{\eta_{j}\right\}_{j}$ ) be a basis for ${ }_{A} \mathcal{H}$ (resp., $\mathcal{H}_{B}$ ). Then, it is easy to see that the bounded vector $\sum_{j} \eta_{j} \underset{B}{\otimes} \overline{\eta_{j}}$ (resp., $\sum_{i} \overline{\xi_{i}} \otimes_{A} \xi_{i}$ ) is independent of the basis and is $A$-A-central, that is, $a\left(\sum_{j} \eta_{j} \underset{B}{\otimes} \overline{\eta_{j}}\right)=\left(\sum_{j} \eta_{j} \underset{B}{\otimes} \overline{\eta_{j}}\right) a$ for all $a \in A$ (resp., $B$-B-central).

Lemma 2.13. Let $\mathcal{K}$ be a right $B$-module with $\operatorname{dim}\left(\mathcal{K}_{B}\right)<\infty$ and $\mathcal{H}$ be a bifinite $A$-B bimodule for $I I_{1}$ factors $A$ and $B$. Then, the inclusion of $I I_{1}$ factors

$$
\begin{aligned}
\mathcal{L}_{B}(\mathcal{K}) & \hookrightarrow \mathcal{L}_{A}\left(\mathcal{K}{\underset{B}{\otimes}}_{\overline{\mathcal{H}}}\right) \hookrightarrow \mathcal{L}_{B}\left(\mathcal{K}{\underset{B}{\otimes}}_{\overline{\mathcal{H}}} \underset{A}{\otimes} \mathcal{H}\right) \\
x & \mapsto x \underset{B}{\otimes} i d_{\overline{\mathcal{H}}}, \quad y \mapsto y \underset{A}{\otimes} i d_{\mathcal{H}}
\end{aligned}
$$

is an instance of basic construction with Jones projection e given (on bounded vectors) by

$$
\mathcal{K} \underset{B}{\otimes} \overline{\mathcal{H}} \underset{A}{\otimes} \mathcal{H} \ni \underset{B}{\xi} \otimes_{\bar{\eta}} \underset{A}{\otimes} \zeta \stackrel{e}{\longmapsto} \frac{1}{\operatorname{dim}_{A} \mathcal{H}} \sum_{i}\left(\xi \underset{B}{\otimes_{B}} \bar{\xi}_{i} \otimes_{A} \xi_{i}\right)\langle\eta, \zeta\rangle_{B} \in \mathcal{K} \underset{B}{\otimes} \overline{\mathcal{H}} \otimes_{A} \mathcal{H}
$$

where $\left\{\xi_{i}\right\}_{i}$ is a basis for ${ }_{A} \mathcal{H}$.

Proof: Set $N=\mathcal{L}_{B}(\mathcal{K}), M=\mathcal{L}_{A}(\mathcal{K} \underset{B}{\otimes} \overline{\mathcal{H}})$ and $M_{1}=\mathcal{L}_{B}(\mathcal{K} \underset{B}{\otimes} \overline{\mathcal{H}} \underset{A}{\otimes} \mathcal{H})$. Let $\left\{\eta_{j}\right\}_{j}$ (resp., $\left.\left\{\sigma_{k}\right\}_{k}\right)$ be a basis for $\mathcal{H}_{B}$ (resp., $\mathcal{K}_{B}$ ). Then, using Proposition 2.12, it is completely routine to check that the map $E_{N}: M \rightarrow N$ given by $E_{N}(x)(\xi)=\left[\operatorname{dim}\left({ }_{A} \mathcal{H}\right)\right]^{-1} \sum_{i, i^{\prime}, k} \sigma_{k}\left\langle\xi_{i^{\prime}},\left\langle\sigma_{k} \underset{B}{\otimes} \bar{\xi}_{i^{\prime}}, x\left(\xi \underset{B}{\otimes} \bar{\xi}_{i}\right)\right\rangle_{A} \xi_{i}\right\rangle_{B}$ for all $\xi \in \mathcal{K}^{o}, x \in M$, is the unique $\operatorname{tr}_{M}$ preserving conditional expectation from $M$ onto $N$. Further, it can also be readily shown that $e \in \mathscr{P}\left(N^{\prime} \cap M_{1}\right)$ and $e\left(x \underset{A}{\otimes} i d_{\mathcal{H}}\right) e=e\left(E_{N}(x) \underset{B}{\otimes} i d_{\overline{\mathcal{H}} \underset{A}{\otimes \mathcal{H}}}\right)$ for all $x \in M$. In view of Proposition 2.11, it just remains to show that $M_{1} e=M e$. Let $x_{1} \in M_{1}$ and consider the map $x: \mathcal{K} \underset{B}{\otimes} \overline{\mathcal{H}} \rightarrow \mathcal{K} \underset{B}{\otimes} \overline{\mathcal{H}}$ given (on bounded vectors) by $x(\xi \underset{B}{\otimes} \bar{\eta})=\sigma_{k} \underset{B}{\otimes} \bar{\xi}_{i^{\prime}} A\left\langle\eta, \eta_{j}\left\langle\sigma_{k}{\underset{B}{B}}_{i^{\prime}} \bar{\xi}_{A} \eta_{j}, x_{1}\left(\xi \underset{B}{\otimes} \bar{\xi}_{i} \otimes_{A} \xi_{i}\right)\right\rangle_{B}\right.$ for all $\xi \in\left(\mathcal{K}_{B}\right)^{o}, \eta \in\left(\mathcal{H}_{B}\right)^{o}$. Clearly, $x \in M$, and it involves nothing more than straightforward verification to show that $\left(x \otimes_{A} i d_{\mathcal{H}}\right) e=x_{1} e$.

We denote the bicategory of bifinite bimodules with $\mathcal{B}$ whose 0 -cells are $I I_{1}$-factors; for $I I_{1}$-factors $A$ and $B$, the objects of the category $\mathcal{B}(B, A)$ are bifinite $A$-B bimodules and morphisms or 2-cells are $A-B$ linear maps between such bimodules (which are automatically bounded). The tensor functor is given by the usual relative tensor product of bimodules and for each $I I_{1}$-factor $A$ the identity object in $\mathcal{B}(A, A)$ is the canonical $A$ - $A$-bimodule $L^{2}(A)$. There is a natural associativity constraint for relative tensor product of bimodules. Further, for an $A$ - $B$-bimodule $\mathcal{H}$, the unit constraints are given by the canonical isomorphisms $L^{2}(A) \underset{A}{\otimes} \mathcal{H} \stackrel{A-B}{\cong} \mathcal{H}$ and $\mathcal{H} \underset{B}{\otimes} L^{2}(B) \stackrel{A-B}{\cong} \mathcal{H}$. Thus, $\mathcal{B}$ has a natural bicategory structure. For the (right) rigid structure on $\mathcal{B}$, for each $A$-B-bimodule $\mathcal{H}$, we $\operatorname{set}\left({ }_{A} \mathcal{H}_{B}\right)^{\#}={ }_{B} \overline{\mathcal{H}}_{A}$ and define the evaluation and coevaluation maps $e_{\mathcal{H}} \in{ }_{B} \mathcal{L}_{B}\left(\overline{\mathcal{H}} \otimes_{A} \mathcal{H}, L^{2}(B)\right)$ and $c_{\mathcal{H}} \in{ }_{A} \mathcal{L}_{A}\left(L^{2}(A), \mathcal{H} \otimes_{B} \overline{\mathcal{H}}\right)$ respectively, (on bounded vectors) by

$$
e_{\mathcal{H}}(\bar{\xi} \underset{A}{\otimes} \eta)=\langle\xi, \eta\rangle_{B} \text { and } c_{\mathcal{H}}(\hat{a})=\sum_{i} a\left(\eta_{j}{\underset{B}{B}}_{\vec{\eta}} \bar{\eta}_{j}\right) \text { for all } \xi, \eta \in \mathcal{H}^{o}, a \in A, b \in B
$$

where $\left\{\eta_{j}\right\}$ is a basis for the right $B$-modple $\mathcal{H}_{B}$. Thus, $\mathcal{B}$ indeed inherits a canonical rigid structure. Finally, the canonical isomorphism ${ }_{A} \mathcal{H}_{B} \stackrel{A}{\cong}{ }_{A} \overline{\overline{\mathcal{H}}}_{B}$ for any bifinite $A$ - $B$-bimodule ${ }_{A} \mathcal{H}_{B}$, equips $\mathcal{B}$ with a pivotal structure. Note that, for $\theta \in{ }_{A} \mathcal{L}_{B}(\mathcal{H}, \mathcal{K})$, it can be easily shown that $\theta^{\#}(\bar{\xi})=\overline{\theta^{*}(\xi)}$ and, hence,

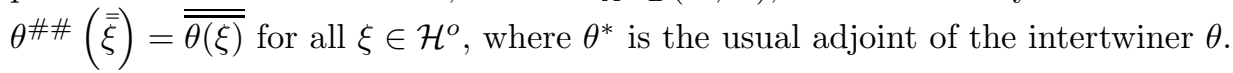

\section{Perturbations of planar algebras}

In this section, we define perturbation of a planar algebra to obtain a new one which has the same filtered algebra structure but the action of Jones projections and conditional expectation tangles differ. As we will 
see, this will turn out to be crucial in the following sections. We must mention here that such objects already appeared in the work of Michael Burns [Bur] while extending Jones theorem (of associating a spherical planar algebra to an extremal subfactor) in the non-extremal case; however, for us, these arose in a purely different context, namely, while detecting the effect of different pivotal structures on the planar algebra associated to a 1-cell in a strict 2-category (as in Gho]).

Definition 3.1. Let $P$ be a planar algebra. An invertible element $z \in P_{+1}$ is said to be a weight of $P$ if $z_{\varepsilon k} \in \mathcal{Z}\left(P_{\varepsilon k}\right)$ for all $\varepsilon k \in$ Col, where
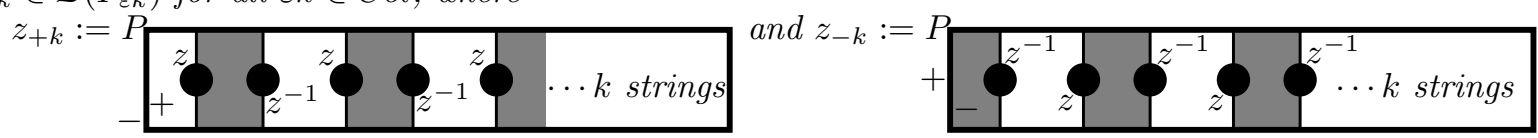

Given a weight $z$ of a planar algebra $P$ and an invertible decomposition $z=a b$ for $a, b$ invertible in $P_{+1}$, we now construct a new planar algebra $P^{(a, b)}$ as follows:

(i) Vector spaces: $P_{\varepsilon k}^{(a, b)}:=P_{\varepsilon k}$ for all $\varepsilon k \in$ Col.

(ii) Actions of tangles: Let $T$ be a tangle and $\hat{T}$ be a standard form representative (see [Gho, $\S 4]$ ) of the isotopy class of $T$. We replace each local maximum and minimum appearing in $\hat{T}$ as in Figure 3.1 and call the resulting semi-labelled tangular diagram $\hat{T}^{(a, b)}$. Define $P_{T}^{(a, b)}:=P_{\hat{T}^{(a, b)}}$. The immediate thing to check
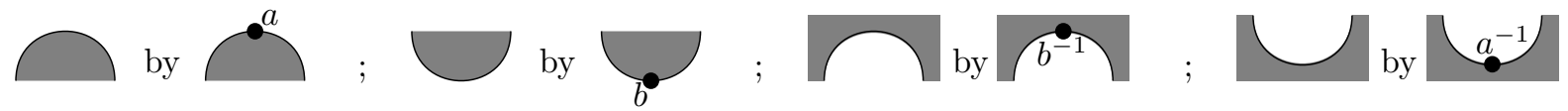

Figure 3.1. Perturbing a planar algebra

is the well-definedness of $P_{T}^{(a, b)}$. Note that the above prescription is invariant under the sliding, wiggling and $360^{\circ}$-rotation moves, applying a finite sequence of which takes one standard form representative to another. Hence, $P_{T}^{(a, b)}$ is well defined and $P^{(a, b)}$ is a planar algebra. As mentioned before, note that $P^{(a, b)}$ has same filtered algebra structure as $P$ whereas the action of Jones projection tangles and conditional expectation tangles differ.

We will refer $P^{(a, b)}$ as the perturbation of $P$ by the decomposition $z=a b$ of the weight $z$.

Remark 3.2. For an invertible decomposition $z=$ ab of a weight $z$ of a planar algebra $P$ and any $\lambda \in \mathbb{C} \backslash\{0\}$, $P^{(a, b)}=P^{\left(\lambda a, \lambda^{-1} b\right)}$. Further, the planar algebras $P^{(a, b)}, P^{(b, a)}, P^{(z, 1)}$, and $P^{(1, z)}$ are all isomorphic. Hence, up to isomorphism, the perturbation of $P$ only depends upon the weight $z$.

To see the first part, observe that the number of local maxima is the same as the number of local minima in a standard form representative of a tangle, which results in cancellation of the scalars appearing in tangle maps due to $\lambda$ in the latter perturbation. In the second part, for instance, the isomorphism $P^{(a, b)} \cong P^{(z, 1)}$ is obtained by the maps

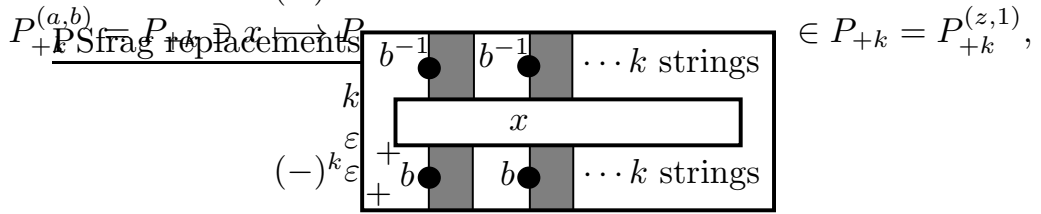

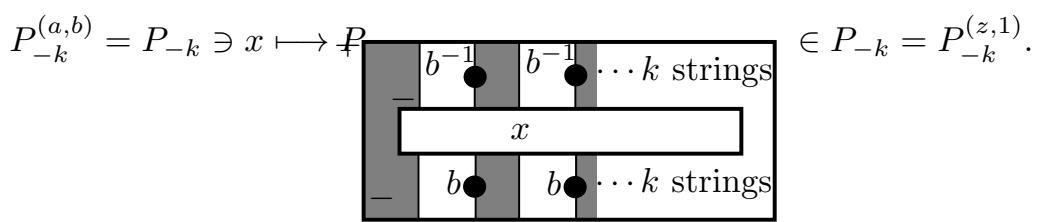

It is straight forward to verify the equivariance of this isomorphism with the actions of the generating tangles in Figure 2.1 and hence all tangles.

A trivial example of a weight of a planar algebra $P$ is a non-zero scalar $\lambda \in \mathbb{C}$. By the above remark, $P^{(\lambda, \mu)}=P^{(\lambda \mu, 1)}=P^{(1, \lambda \mu)}=P^{(\mu, \lambda)}$ for all non-zero scalars $\lambda$ and $\mu$. We will usually refer to such perturbations as scalar perturbations.

A perturbation of a planar algebra with modulus need not have modulus (except for perturbations by scalar weights). However, if the planar algebra is connected then so are its perturbations, but the moduli 
of the perturbations might vary. For instance, for a planar algebra $P$ with modulus $\left(\delta_{-}, \delta_{+}\right)$, the scalar perturbation $P^{(\lambda, 1)}$ has modulus $\left(\lambda^{-1} \delta_{-}, \lambda \delta_{+}\right)$.

Definition 3.3. The normalized planar algebra associated to a planar algebra $P$ with modulus $\left(\delta_{-}, \delta_{+}\right)$is its scalar perturbation by the weight $\sqrt{\frac{\delta_{-}}{\delta_{+}}}$.

Note that the normalization of $P$ is a unimodular planar algebra. Although scalar perturbations change the modulus, the index however remains the same; in the last section, we will come across an example of a perturbation class whose normalized planar algebras realize all indices greater than or equal to 4 .

Definition 3.4. A connected planar algebra $P$ is said to be spherical if the actions of 0-tangles in its normalization are invariant under spherical isotopy.

Note that this property is equivalent to demanding that the normalized left and right picture traces on $P_{+1}$ are identical. The relevance of the above definition will become clear in the section on bimodule planar algebras, where we establish a correspondence between sphericality and extremality. We must also point out that a non-unimodular planar algebra could be spherical according to the above definition which is not allowed in Jones' original definition in Jon2.

In general, a perturbation of a $*$-planar algebra need not be a $*$-planar algebra. However, for certain specific weights, the perturbations also turn out to be $*$-planar algebras. For instance, if $P$ is a $*$ - (resp., positive) planar algebra, it is routine to verify that a perturbation of the type $P^{\left(a, \lambda a^{*}\right)}$ for non-zero real (resp., positive) scalar $\lambda$ becomes a $*$ - (resp., positive) planar algebra with $*$-structure coming from the original one.

\section{Weights and Pivotality}

In this section, we mention how one can canonically associate a pivotal $\mathbb{C}$-linear strict 2-category to a planar algebra; conversely, from Gho, we recall how to associate a planar algebra to a 1-cell in a pivotal $\mathbb{C}$-linear strict 2-category. We then establish a relation between weights and perturbations of planar algebras and pivotal structures on bicategories.

4.1. Planar algebras to bicategories. Let $P$ be a planar algebra. From $P$, we first describe a $\mathbb{C}$-linear strict 2-category $\mathcal{B}$ and see that it inherits canonical rigid and pivotal structures. Set $\mathcal{B}_{0}=\{+,-\}$; for $\varepsilon, \eta \in \mathcal{B}_{0}$, set $o b(\mathcal{B}(\varepsilon, \eta))=2 \mathbb{N}_{0}+\delta_{\varepsilon \neq \eta}$. To avoid confusion, we will write an object $k \in o b(\mathcal{B}(\varepsilon, \eta))$ as $\varepsilon k$ (whence $\eta=(-)^{k} \varepsilon$ ). For two objects $\varepsilon k, \varepsilon l$, set $\operatorname{Mor}(\varepsilon k, \varepsilon l)=P_{(-)^{k} \varepsilon\left(\frac{k+l}{2}\right)}=P_{(-)^{l} \varepsilon\left(\frac{k+l}{2}\right)}$. Composition of morphisms be given by the bilinear map

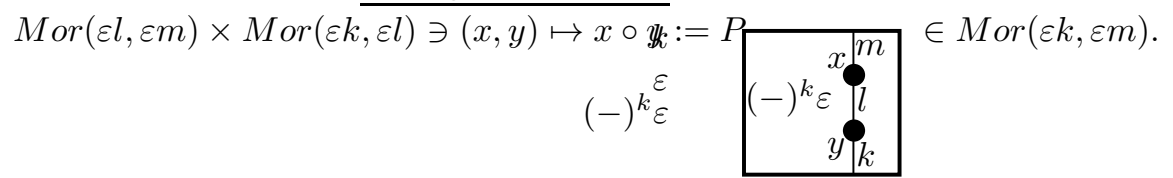

For each 1-cell $\varepsilon k$, the identity morphism is given by $P_{1_{\varepsilon k}}$. The tensor functor $\otimes: \mathcal{B}(\eta, \sigma) \times \mathcal{B}(\varepsilon, \eta) \rightarrow \mathcal{B}(\varepsilon, \sigma)$ is defined by $\eta l \otimes \varepsilon k:=\varepsilon(k+l)$ and

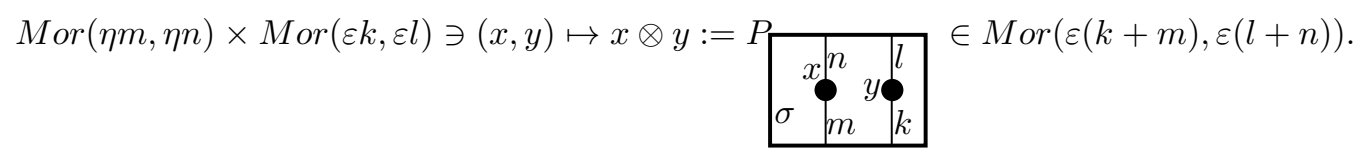

For each 0 -cell $\varepsilon$, set the identity object $1_{\varepsilon}=\varepsilon 0 \in o b(\mathcal{B}(\varepsilon, \varepsilon))$. With the above structure, it is easily seen that $\mathcal{B}$ is a strict 2 -category. We now describe a (right) rigid structure $\#$ on $\mathcal{B}$ as follows:

0 -cells: $\varepsilon^{\#}:=\varepsilon$.

1-cells: $(\varepsilon k)^{\#}=(-)^{k} \varepsilon k$.

The evaluation map $e_{\varepsilon k}:(\varepsilon k)^{\#} \otimes \varepsilon k=\varepsilon 2 k \rightarrow \varepsilon 0$ (resp., coevaluation map $c_{\varepsilon k}:(-)^{k} \varepsilon 0 \rightarrow \varepsilon k \otimes(\varepsilon k)^{\#}=$ $\left.(-)^{k} \varepsilon 2 k\right)$ will be given by $e_{\varepsilon k}:=P_{\varepsilon_{k}}$ (resp., $c_{\varepsilon k}:=P \underbrace{k}_{(-)^{k} \varepsilon})$. Thus, we obtain a weak functor

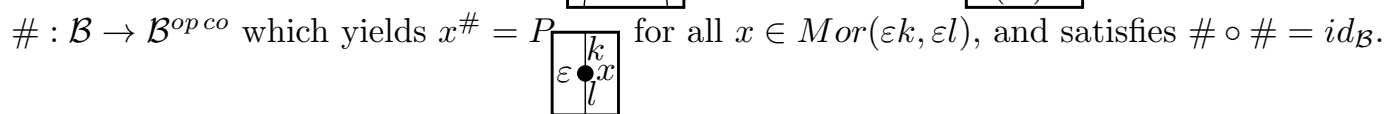


This strict 2-category $\mathcal{B}$ also inherits a canonical pivotal structure $a: i d_{\mathcal{B}} \rightarrow \# \circ \#=i d_{\mathcal{B}}$, which is identity on $\mathcal{B}_{0}$, the objects $a_{\varepsilon}:=\varepsilon 0 \in o b(\mathcal{B}(\varepsilon, \varepsilon))$ and the morphisms $a_{\varepsilon k}:=i d_{\varepsilon k}: \varepsilon k \rightarrow \varepsilon k$. In fact, we have a correspondence between the pivotal structures on $\mathcal{B}$ and the weights of $P$.

Proposition 4.1. There is a one-to-one correspondence between weights of a planar algebra $P$ and pivotal structures on the strict 2 -category $\mathcal{B}$ associated to $P$ as above.

Proof: Given a weight $z$ of $P$, define $a_{\varepsilon k}:=z_{(-)^{k} \varepsilon k} \in \operatorname{Mor}(\varepsilon k, \varepsilon k)$. For naturality of $a$, consider $f \in$ $\operatorname{Mor}(\varepsilon k, \varepsilon l)$. Note that $a_{\varepsilon l}^{-1} \circ f \circ a_{\varepsilon k}=z_{(-)^{k} \varepsilon \frac{k+l}{2}}^{-1} \circ f \circ z_{(-)^{k} \varepsilon \frac{k+l}{2}}=f$ where the first equality readily follows from pictures and the second one holds because $z$ 's are central. Also, the tensor condition $a_{(-)^{k} \varepsilon l} \otimes a_{\varepsilon k}=a_{\varepsilon(k+l)}$ is easy to verify. Setting $a_{\varepsilon}=1_{\varepsilon}$, we have a weak transformation $a: i d_{\mathcal{B}} \rightarrow \# \circ \#=i d_{\mathcal{B}}$. This proves that $a$ gives a pivotal structure.

Conversely, if $a: i d_{\mathcal{B}} \rightarrow \# \circ \#=i d_{\mathcal{B}}$ is a pivotal structure, set $z=a_{-1} \in \operatorname{Mor}(-1,-1)=P_{+1}$. Then, the tensor property of $a$, namely $a_{(-)^{k} \varepsilon l} \otimes a_{\varepsilon k}=a_{\varepsilon(k+l)}$, along with naturality of $a$ and Proposition $[2.9$ (2) implies that $z_{\varepsilon k} \in \mathcal{Z}\left(P_{\varepsilon k}\right)$ and hence $z$ is a weight of $P$.

4.2. Bicategories to planar algebras. We first briefly recall from Gho the planar algebra associated to any 1-cell in a pivotal $\mathbb{C}$-linear strict 2-category $\mathcal{B}$. Let $X \in o b(\mathcal{B}(-,+))$ for $\{+,-\} \subset \mathcal{B}_{0}, \#: \mathcal{B} \rightarrow \mathcal{B}^{\text {opco }}$ be a right rigid structure with respect to evaluation and coevaluation $e$ and $c$ respectively, and $a: i d_{B} \rightarrow \# \circ \#$ be a pivotal structure. The ingredients of the planar algebra $P$ associated to $(\mathcal{B}, \#, a, X)$ are as follows:

Vectors spaces: $P_{\varepsilon k}:=\operatorname{End}\left(X_{\varepsilon k}\right)$, where

$$
X_{\varepsilon k}:=\left\{\begin{array}{c}
X \otimes X^{\#} \otimes X \otimes X^{\#} \otimes X \otimes \cdots k \text { many tensor factors if } \varepsilon=+ \\
X^{\#} \otimes X \otimes X^{\#} \otimes X \otimes X^{\#} \otimes \cdots k \text { many tensor factors if } \varepsilon=-
\end{array}\right.
$$

if $k \geq 1$ and $X_{\varepsilon 0}:=1_{\varepsilon} \in o b(\mathcal{B}(\varepsilon, \varepsilon))$.

Actions of tangles: Given a tangle $T:\left(\varepsilon_{1} k_{1}, \ldots \varepsilon_{b} k_{b}\right) \rightarrow \varepsilon_{0} k_{0}$ and elements $x_{i} \in P_{\varepsilon_{i} k_{i}}, 1 \leq i \leq b$, the action $P_{T}\left(x_{1}, \ldots, x_{b}\right)$ is given by $(i)$ choosing a standard form representative $T_{1}$ in the isotopy class of $T$ labelled with $x_{i}$ in the $i$-th internal box, $(i i)$ cutting $T_{1}$ into horizontal stripes so that each stripe should have at most one local maximum, minimum or (labelled) internal rectangle, (iii) assigning a 2-cell to each horizontal stripe as prescribed in Figure 4.1 and $(i v)$ successively composing these 2-cells with the one coming from the bottom stripe being the rightmost in the composition.

$$
\begin{aligned}
& \left.\left.\right|^{k}\right|^{l} \leadsto i d_{X_{(-)^{k}}} \otimes\left(e_{X^{\#}} \circ\left(a_{X} \otimes i d_{X^{\#}}\right)\right) \otimes i d_{X_{+l}} \in \operatorname{Mor}\left(X_{(-)^{k}(k+l+2)}, X_{(-)^{k}(k+l)}\right) \\
& |k|_{l} \leadsto i d_{X_{(-)^{k} k}} \otimes c_{X} \otimes i d_{X_{+l}} \in \operatorname{Mor}\left(X_{(-)^{k}(k+l)}, X_{(-)^{k}(k+l+2)}\right) \\
& \left.{ }^{k}\right|^{l} \leadsto i d_{X_{(-)^{k+1}}} \otimes e_{X} \otimes i d_{X_{-l}} \in \operatorname{Mor}\left(X_{(-)^{k+1}(k+l+2)}, X_{(-)^{k+1}(k+l)}\right) \\
& \prod_{l} \leadsto i d_{X_{(-)^{k+1} k}} \otimes\left(\left(i d_{X^{\#}} \otimes a_{X}^{-1}\right) \circ c_{X^{\#}}\right) \otimes i d_{X_{-l}} \in \operatorname{Mor}\left(X_{(-)^{k+1}(k+l)}, X_{(-)^{k+1}(k+l+2)}\right)
\end{aligned}
$$

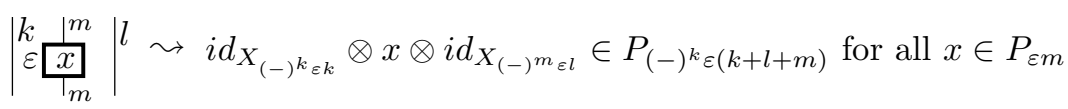

FiguRE 4.1. Prescription for bicategory planar algebra

Proposition 4.2. Let $a$ and $\tilde{a}$ be two pivotal structures on $\mathcal{B}$ as above, $P$ and $\tilde{P}$ be the planar algebras associated to a 1-cell $X$ with respect to $a$ and $\tilde{a}$ respectively. Then, $P$ and $\tilde{P}$ are perturbations of each other.

Proof: Set $z=a_{X}^{-1} \circ \tilde{a}_{X} \in P_{+1}=\operatorname{End}(X)$. We assert that $z$ is a weight of $P$ and $P^{(z, 1)}=\tilde{P}$. It follows from the definition of action of tangles and [Gho, Lemma 4.2] that $z^{\#}=P\left[z\right.$. Note that $z_{+k}$ (as in Definition 
3.1) is given by

$$
\begin{aligned}
z_{+k} & =z \otimes\left(z^{-1}\right)^{\#} \otimes z \otimes \cdots k \text {-tensors } \\
& =\left(a_{X} \otimes a_{X \#} \otimes a_{X} \otimes \cdots\right)^{-1} \circ\left(\tilde{a}_{X} \otimes \tilde{a}_{X \#} \otimes \tilde{a}_{X} \otimes \cdots\right) \\
& =a_{X_{+k}}^{-1} \circ \tilde{a}_{X_{+k}} \in \mathcal{Z}\left(P_{+k}\right),
\end{aligned}
$$

where in the second (resp., last) equality, we have used the pivotal property of $a$ and $\tilde{a}$ as in Proposition 2.9 (resp., Corollay 2.10). And on similar lines one establishes that $z_{-k} \in \mathcal{Z}\left(P_{-k}\right)$. This shows that $z$ is indeed a weight of $P$. Now, it is a matter of routine verification that $P_{T}^{(z, 1)}=\tilde{P}_{T}$ for a set of generating tangles as in Figure 2.1. Thus, we conclude that $P^{(z, 1)}=\tilde{P}$.

\section{Bimodule Planar algebras}

5.1. Planar algebra associated to a bimodule. In this subsection, we associate a 'bimodule planar algebra' to a bifinite bimodule with a natural correspondence between extremality and sphericality. This will pave the way for us to associate a unimodular bimodule planar algebra to a finite index subfactor, giving an extension of Jones' Theorem [Jon2, Theorem 4.2.1] to an arbitrary finite index subfactor (not necessarily extremal).

Definition 5.1. A finite dimensional, connected, positive $C^{*}$-planar algebra is called bimodule planar algebra.

Let ${ }_{A} \mathcal{H}_{B}$ be a finite index bimodule for $I I_{1}$ factors $A$ and $B$. Before stating the next theorem, we set up some notations that will be used throughout this section:

$\mathcal{H}_{+0}:=L^{2}(A), \mathcal{H}_{-0}:=L^{2}(B)$ and for $k \geq 1, \mathcal{H}_{\varepsilon k}$ is the tensor product (over $A$ or $B$ ) of $k$-many modules $\mathcal{H}$ and $\overline{\mathcal{H}}$ alternately with $\mathcal{H}$ (resp., $\overline{\mathcal{H}}$ ) being the left-most module if $\varepsilon=+$ (resp., - ).

Theorem 5.2. Let ${ }_{A} \mathcal{H}_{B}$ be a finite index bimodule for $I I_{1}$-factors $A$ and $B$. Then, (i) $P$ defined by

$$
P_{\varepsilon k}= \begin{cases}{ }_{A} \mathcal{L}_{A}\left(\mathcal{H}_{+k}\right) \text { or }{ }_{A} \mathcal{L}_{B}\left(\mathcal{H}_{+k}\right), & \text { if } \varepsilon=+, \\ B & \mathcal{L}_{B}\left(\mathcal{H}_{-k}\right) \text { or }{ }_{B} \mathcal{L}_{A}\left(\mathcal{H}_{-k}\right), \text { if } \varepsilon=-,\end{cases}
$$

according as $k$ is even or odd, has a unique bimodule planar algebra structure with $*$-structure coming from the usual adjoints of intertwiners, satisfying:

(a) action of multiplication tangles matches with the composition of operators in the intertwiner spaces,

(b) $P_{R I_{\varepsilon k}}= \begin{cases}P_{\varepsilon k} \ni T \mapsto T \underset{A}{\otimes} i d_{\mathcal{H}} \in P_{\varepsilon(k+1)}, & \text { if either } \varepsilon=+ \text { and } k \text { is even, or } \varepsilon=- \text { and } k \text { is odd, } \\ P_{\varepsilon k} \ni T \mapsto T \underset{B}{\otimes} i d_{\overline{\mathcal{H}}} \in P_{\varepsilon(k+1)}, & \text { otherwise, }\end{cases}$

(c) $P_{L I_{\varepsilon k}}= \begin{cases}P_{\varepsilon k} \ni T \mapsto i d_{\overline{\mathcal{H}}}{\underset{A}{A}}_{A} T \in P_{-\varepsilon(k+1)}, & \text { if } \varepsilon=+, \\ P_{\varepsilon k} \ni T \mapsto i d_{\mathcal{H}} \otimes_{B}^{\otimes} T \in P_{-\varepsilon(k+1)}, & \text { if } \varepsilon=- \text { and }\end{cases}$

(d) $P_{E_{+1}}$ (resp., $P_{E_{-1}}$ ) is given by

$$
\begin{aligned}
& \mathcal{H}^{o} \underset{B}{\otimes} \overline{\mathcal{H}}^{o} \ni \xi \underset{B}{\otimes} \bar{\eta} \stackrel{A-A}{\longmapsto} \sum_{j} A\langle\eta, \xi\rangle\left(\eta_{j} \underset{B}{\otimes} \bar{\eta}_{j}\right) \in \mathcal{H}^{o} \underset{B}{\otimes} \overline{\mathcal{H}}^{o} \\
& \text { (resp., } \left.\overline{\mathcal{H}}^{o} \underset{A}{\otimes} \mathcal{H}^{o} \ni \bar{\eta} \underset{A}{\otimes} \stackrel{B-B}{\longmapsto} \sum_{i}\langle\eta, \xi\rangle_{B}\left(\bar{\xi}_{i}{\underset{A}{\otimes}}_{i}\right) \in \overline{\mathcal{H}}^{o}{\underset{A}{\otimes}}_{\mathcal{H}^{o}}\right)
\end{aligned}
$$

where $\left\{\xi_{i}\right\}_{i}$ (resp., $\left\{\eta_{j}\right\}_{j}$ ) is any basis for ${ }_{A} \mathcal{H}$ (resp., $\mathcal{H}_{B}$ ). (We will refer $P$ (resp., normalized $P$ ) as the bimodule (resp., normalized or unimodular) planar algebra associated to ${ }_{A} \mathcal{H}_{B}$ ).

(ii) $P$ (as in (i)) is spherical if and only if ${ }_{A} \mathcal{H}_{B}$ is extremal (as in \$2.3).

(iii) If $B^{\prime}:=\mathcal{L}_{B}(\mathcal{H})$, then the normalized planar algebras associated to ${ }_{A} \mathcal{H}_{B}$ and ${ }_{A} L^{2}\left(B^{\prime}\right)_{B^{\prime}}$ are isomorphic as $*$-planar algebras.

Proof: (i) The planar algebra (in [Gho]) associated to the 1-cell ${ }_{A} \mathcal{H}_{B}$ in the pivotal bicategory of bifinite bimodules $(\S 2.3)$ has same vector spaces as of $P$; we provide $P$ with the same planar algebra structure, the prescription for the actions of tangles for which is given in Figure 4.1 It is clear from this prescription that $P$ satisfies the conditions $(a)-(d)$. Since $P$ is connected, the fact that $P$ with the above mentioned 
*-structure is a $C^{*}$-planar algebra, can be verified readily by checking the $*$-condition (2.1) for the tangles $M_{\varepsilon k}, R I_{\varepsilon k}, L I_{\varepsilon k}, E_{\varepsilon k}$. It now remains to show that the $C^{*}$-planar algebra $P$ is positive. For this, it is enough to show that the tangle maps $P_{R E_{\varepsilon k}}$ and $P_{L E_{\varepsilon k}}$ are positive definite for all $\varepsilon k \in C o l$. We prove this only for $P_{R E_{+2 k}}$; the others can be verified using similar arguments. For each $x \in P_{+2 k}={ }_{A} \mathcal{L}_{A}\left(\mathcal{H}_{+2 k}\right)$ and $\zeta \in \mathcal{H}_{+(2 k-1)}^{o}$, we have

$$
\begin{aligned}
P_{R E_{+2 k}}(x)(\zeta) & =\sum_{i}\left(\left(i d_{\mathcal{H}_{+(2 k-1)}} \underset{B}{\otimes} e_{\mathcal{H}}\right) \circ\left(x \underset{A}{\otimes} i d_{\mathcal{H}}\right)\right)\left(\zeta \underset{B}{\otimes} \bar{\xi}_{i} \underset{A}{\otimes} \xi_{i}\right) \\
& =\sum_{i, i^{\prime}, k}\left(i d_{\mathcal{H}_{+(2 k-1)}} \underset{B}{\otimes} e_{\mathcal{H}}\right)\left(\gamma_{k} \underset{B}{\otimes} \bar{\xi}_{i}\left\langle\gamma_{k}{\underset{B}{B}}_{\bar{i}^{\prime}}, x\left(\zeta \underset{B}{\otimes} \bar{\xi}_{i}\right)\right\rangle_{A}{\underset{A}{\otimes}}_{\xi_{i}}\right) \\
& =\sum_{i, i^{\prime}, k} \gamma_{k}\left\langle\xi_{i^{\prime}},\left\langle\gamma_{k} \underset{B}{\otimes} \bar{\xi}_{i^{\prime}}, x\left(\zeta{\underset{B}{B}}_{i} \bar{\xi}_{i}\right)\right\rangle_{A} \xi_{i}\right\rangle_{B},
\end{aligned}
$$

where $\left\{\gamma_{k}\right\}_{k}$ is a basis for $\left(\mathcal{H}_{+(2 k-1)}\right)_{B}$. This gives

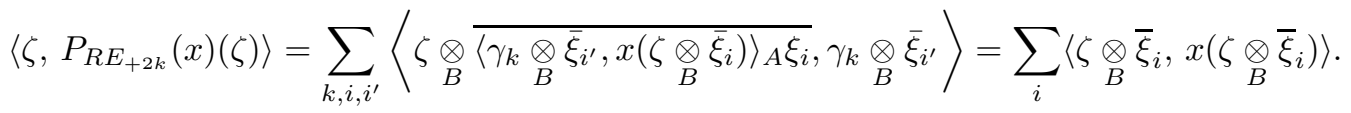

Thus, $P_{R E_{+2 k}}(x)$ is positive semi-definite. In addition, the above also gives

$$
\sum_{k}\left\langle\gamma_{k}, P_{R E_{+2 k}}(x)\left(\gamma_{k}\right)\right\rangle=\sum_{k, i}\left\langle\gamma_{k} \underset{B}{\otimes} \bar{\xi}_{i}, x\left(\gamma_{k} \underset{B}{\otimes} \bar{\xi}_{i}\right)\right\rangle=\operatorname{dim}\left(\mathcal{H}_{+2 k}\right)_{A} \operatorname{tr}_{C}(x),
$$

where $C:=\mathcal{L}_{A}\left(\mathcal{H}_{+2 k}\right)$ and the last equality is a consequence of Proposition 2.12. This proves that $P_{R E_{+2 k}}$ is also faithful. Thus, $P$ is a bimodule planar algebra; and for uniqueness, we appeal again to Remark 2.2 .

(ii) It is enough to prove that the normalized left and the right picture traces on $P_{+1}$ are given by the unique traces on the $I I_{1}$-factors $A^{\prime}:={ }_{A} \mathcal{L}(\mathcal{H})$ and $B^{\prime}:=\mathcal{L}_{B}(\mathcal{H})$ respectively. We will only exhibit a proof for the left one. First, note that

$$
\begin{gathered}
P \longrightarrow=e_{\mathcal{H}} \circ\left(i d_{\overline{\mathcal{H}}} \otimes a_{\mathcal{H}}^{-1}\right) \circ c_{\overline{\mathcal{H}}}=\sum_{i}\left\langle\xi_{i}, \xi_{i}\right\rangle_{\hat{B}}=\operatorname{dim}\left({ }_{A} \mathcal{H}\right) 1_{P_{-0}} \text { and } \\
P=e_{\overline{\mathcal{H}}} \circ\left(a_{\mathcal{H}} \otimes i d_{\overline{\mathcal{H}}}\right) \circ c_{\mathcal{H}}=\sum_{j}\left\langle\eta_{j}, \eta_{j}\right\rangle^{\wedge}=\operatorname{dim}\left(\mathcal{H}_{B}\right) 1_{P_{+0}}
\end{gathered}
$$

where $\left\{\xi_{i}\right\}_{i}$ (resp., $\left\{\eta_{j}\right\}_{j}$ ) is a basis for ${ }_{A} \mathcal{H}$ (resp. $\mathcal{H}_{B}$ ). So, the modulus of the planar algebra $P$ is $\left(\operatorname{dim}_{A} \mathcal{H}, \operatorname{dim}_{B}\right)$. Further, for each $x \in P_{+1}={ }_{A} \mathcal{L}_{B}(\mathcal{H})$,

$$
\mathcal{H}_{-0}:=L^{2}(B) \ni \hat{1} \stackrel{P_{L E_{+}}(x)}{\longmapsto} \sum_{i}\left\langle\xi_{i}, x \xi_{i}\right\rangle_{B}=\sum_{i}\left\langle\xi_{i}, x \xi_{i}\right\rangle \hat{1}=\operatorname{dim}\left({ }_{A} \mathcal{H}\right) \operatorname{tr}_{A^{\prime}}(x) \hat{1} \in L^{2}(B)=: \mathcal{H}_{-0} .
$$

The first equality follows from the fact that $\sum_{i}\left\langle\xi_{i}, x \xi_{i}\right\rangle_{B}$ commutes with $B$ and the second one comes again from Proposition 2.12

(iii) Note that the planar algebra associated to ${ }_{A} \mathcal{H}_{B}$ may very well be non-unimodular but the index of $P$ is same as that of ${ }_{A} \mathcal{H}_{B}$. Set $\delta_{-}=\operatorname{dim}\left({ }_{A} \mathcal{H}\right), \delta_{+}=\operatorname{dim}\left(\mathcal{H}_{B}\right)$ and $\delta=\sqrt{\delta_{+} \delta_{-}}$. Let $Q$ denote the bimodule planar algebra associated to ${ }_{A} L^{2}\left(B^{\prime}\right)_{B^{\prime}}$. So, $\tilde{P}:=P^{\left(\sqrt{\frac{\delta_{-}}{\delta_{+}}}, 1\right)}$ and $\tilde{Q}:=Q^{(\delta, 1)}$ are the normalizations of $P$ and $Q$ respectively. To establish an isomorphism between $\tilde{P}$ and $\tilde{Q}$, we consider the following $B^{\prime}-B^{\prime}$ linear 
maps $u: \mathcal{H} \underset{B}{\otimes} \overline{\mathcal{H}} \rightarrow \mathcal{K}:=L^{2}\left(B^{\prime}\right)$ and $w: \mathcal{H} \underset{B}{\otimes} \overline{\mathcal{H}} \rightarrow \overline{\mathcal{K}}$ given by

$$
\begin{aligned}
& \mathcal{H}^{o} \underset{B}{\otimes} \overline{\mathcal{H}}^{o} \ni \underset{B}{\otimes} \bar{\eta}_{B^{\prime}}^{u}\langle\eta, \xi\rangle \hat{1} \in \mathcal{K} \text { implying } \mathcal{K} \ni \hat{1} \stackrel{u^{*}}{\mapsto} \delta_{+}^{-1} \sum_{j}\left(\eta_{j} \underset{B}{\otimes} \bar{\eta}_{j}\right) \in \mathcal{H}^{o}{\underset{B}{\otimes}}_{\mathcal{H}^{o}} \text { and }
\end{aligned}
$$

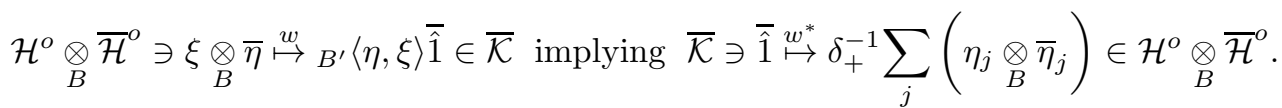

Clearly, $u$ and $w$ are co-isometries; further, $\operatorname{index}\left({ }_{B^{\prime}} \mathcal{H} \underset{B}{\otimes} \overline{\mathcal{H}}_{B^{\prime}}\right)=1$ implies $u$ and $w$ are unitaries as well. Let $v: \mathcal{H} \underset{B}{\otimes} \overline{\mathcal{H}} \rightarrow \mathcal{K} \underset{B^{\prime}}{\otimes} \overline{\mathcal{K}}$ be the $B^{\prime}-B^{\prime}$ linear unitary obtained from $u$ after composing with the unitary taking $\hat{1} \in \mathcal{K}$ to $\left(\hat{1} \underset{B^{\prime}}{\otimes} \overline{\hat{1}}\right) \in \mathcal{K} \underset{B^{\prime}}{\otimes} \overline{\mathcal{K}}$. Define $\varphi: \tilde{P} \rightarrow \tilde{Q}$ in the following way:

$$
\tilde{P}_{+2 l}={ }_{A} \mathcal{L}_{A}\left(\mathcal{H}_{+2 l}\right) \ni x \stackrel{\varphi_{+2 l}}{\longrightarrow} A d_{v_{l}}(x) \in{ }_{A} \mathcal{L}_{A}\left(\mathcal{K}_{+2 l}\right)=\tilde{Q}_{+2 l} \text { for } l \geq 1,
$$

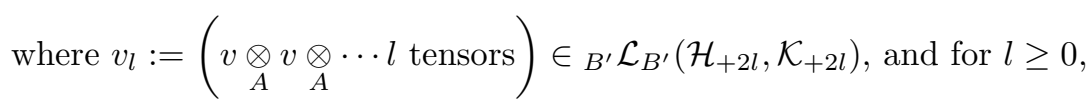

$$
\begin{aligned}
& \tilde{P}_{+(2 l+1)}={ }_{A} \mathcal{L}_{B}\left(\mathcal{H}_{+(2 l+1)}\right) \ni x \stackrel{\varphi_{+(2 l+1)}}{\longmapsto} A d_{\left(v_{l} \otimes u\right)}\left(x \underset{B}{\otimes} i d_{\overline{\mathcal{H}}}\right) \in{ }_{A} \mathcal{L}_{B^{\prime}}\left(\mathcal{K}_{+(2 l+1)}\right)=\tilde{Q}_{+(2 l+1)}, \\
& \tilde{P}_{-(2 l+1)}={ }_{B} \mathcal{L}_{A}\left(\mathcal{H}_{-(2 l+1)}\right) \ni x \stackrel{\varphi_{-(2 l+1)}}{\longmapsto} A d_{\left(w \underset{A}{\left.\otimes v_{l}\right)}\right.}\left(i d_{\mathcal{H}} \underset{B}{\otimes} x\right) \in{ }_{B^{\prime}} \mathcal{L}_{A}\left(\mathcal{K}_{-(2 l+1)}\right)=\tilde{Q}_{-(2 l+1)}, \\
& \tilde{P}_{-(2 l+2)}={ }_{B} \mathcal{L}_{B}\left(\mathcal{H}_{-(2 l+2)}\right) \ni x \stackrel{\varphi_{-(2 l+2)}}{\longmapsto} A d_{\left(w \underset{A}{\otimes} v_{l} \underset{A}{\otimes u}\right)}\left(i d_{\mathcal{H}} \underset{B}{\otimes} \underset{A}{\otimes} i d_{\overline{\mathcal{H}}}\right) \in{ }_{B^{\prime}} \mathcal{L}_{B^{\prime}}\left(\mathcal{K}_{-(2 l+2)}\right)=\tilde{Q}_{-(2 l+2)} .
\end{aligned}
$$

Clearly, each $\varphi_{\varepsilon k}$ is an injective $*$-algebra homomorphism and each $\varphi_{+2 l}$ is surjective. To see that $\varphi_{+(2 l+1)}$ (resp., $\varphi_{-(2 l+1)}$ ) is surjective, note that

$$
\begin{aligned}
& A d_{\left(v_{l} \otimes u\right)^{*}}\left({ }_{A} \mathcal{L}_{B^{\prime}}\left(\mathcal{K}_{+(2 l+1)}\right)\right)={ }_{A} \mathcal{L}_{B^{\prime}}\left(\mathcal{H}_{+(2 l+1)} \underset{B}{\otimes} \overline{\mathcal{H}}\right)=\left\{x \underset{B}{\otimes} i d_{\overline{\mathcal{H}}}: x \in{ }_{A} \mathcal{L}_{B}\left(\mathcal{H}_{+(2 l+1)}\right)\right\} \\
& \left(\text { resp., } A d_{\left(w \underset{A}{\left.\otimes v_{l}\right)^{*}}\right.}\left({ }_{B^{\prime}} \mathcal{L}_{A}\left(\mathcal{K}_{-(2 l+1)}\right)\right)={ }_{B^{\prime}} \mathcal{L}_{A}\left(\mathcal{H}{\underset{B}{\otimes}}_{\mathcal{H}_{-(2 l+1)}}\right)=\left\{i d_{\mathcal{H}}{\underset{B}{B}}_{x} x: x \in{ }_{B} \mathcal{L}_{A}\left(\mathcal{H}_{-(2 l+1)}\right)\right\}\right)
\end{aligned}
$$

where the second equality follows from Lemma 2.13 since ${ }_{B} \overline{\mathcal{H}}_{B^{\prime}}\left(\right.$ resp., ${ }_{B^{\prime}} \mathcal{H}_{B}$ ) has index 1 . Same arguments also imply $\varphi_{-(2 l+2)}$ is surjective. Thus, $\varphi$ is an isomorphism preserving actions of multiplication tangles.

Actions of inclusion tangles: We only show that $\varphi$ is equivariant with respect to the action of $R I_{+k}$; the same for $R I_{-k}$ and $L I_{\varepsilon k}$ can be verified along similar lines. For $x \in \tilde{P}_{+(2 l+1)}$, note that

$$
\left[A d_{v_{l} \otimes u}^{\otimes u}\left(x \underset{B}{\otimes} i d_{\overline{\mathcal{H}}}\right)\right] \underset{B^{\prime}}{\otimes} i d_{\overline{\mathcal{K}}}=A d_{v_{l} \otimes \otimes_{B^{\prime}}^{\otimes}}^{\otimes} i d_{\overline{\mathcal{K}}}\left(x \underset{B}{\otimes} i d_{\overline{\mathcal{H}}} \underset{B^{\prime}}{\otimes} i d_{\overline{\mathcal{K}}}\right)=A d_{v_{l} \underset{A}{\otimes} \circ 0}\left(x \underset{B}{\otimes} i d_{\overline{\mathcal{H}}} \underset{B^{\prime}}{\otimes} i d_{\overline{\mathcal{K}}}\right)=A d_{v_{l} \otimes v}^{\otimes v}\left(x \underset{B}{\otimes} i d_{\overline{\mathcal{H}}}\right),
$$

where $\mathcal{H} \underset{B}{\otimes} \overline{\mathcal{H}} \underset{B^{\prime}}{\otimes} \overline{\mathcal{K}} \ni \underset{B}{\otimes} \underset{B^{\prime}}{\otimes} \underset{\hat{1}}{\stackrel{\theta}{\mapsto}}{\underset{B}{B}}_{B} \bar{\eta} \in \mathcal{H} \underset{B}{\otimes} \overline{\mathcal{H}}$ is a $B^{\prime}-B^{\prime}$ unitary intertwiner. This implies $\varphi\left(\tilde{P}_{R I_{+(2 l+1)}}(x)\right)=$ $\tilde{Q}_{R I_{+(2 l+1)}}(\varphi(x))$. Equivariance of $\varphi$ with respect to the action of $R I_{+2 l}$ is even more easier.

Action of $E_{ \pm 1}$ : From condition $(d)$ of part $(i)$ and definition of perturbation, we obtain

$$
\begin{aligned}
\varphi\left(\tilde{P}_{E_{+1}}\right)\left(\hat{x} \underset{B^{\prime}}{\otimes} \overline{\hat{y}}\right) & =\frac{1}{\delta_{+}} \sum_{j} v \tilde{P}_{E_{+1}}\left(x y^{*} \eta_{j} \underset{B}{\otimes} \bar{\eta}_{j}\right)=\frac{1}{\delta_{+}} \sqrt{\frac{\delta_{-}}{\delta_{+}}} \sum_{j, j^{\prime}} v\left({ }_{A}\left\langle x y^{*} \eta_{j}, \eta_{j}\right\rangle \eta_{j^{\prime}} \otimes \overline{\eta_{j^{\prime}}}\right) \\
& =\frac{1}{\delta_{+}} \sqrt{\frac{\delta_{-}}{\delta_{+}}} \sum_{j, j^{\prime}} E_{A}\left({ }_{B^{\prime}}\left\langle x y^{*} \eta_{j}, \eta_{j}\right\rangle\right) \eta_{j^{\prime}} \otimes \overline{\eta_{j^{\prime}}}=\frac{1}{\delta_{+}} \sqrt{\frac{\delta_{-}}{\delta_{+}}} E_{A}\left(x y^{*}\right)\left(\operatorname{dim} \mathcal{H}_{B}\right)^{2} \hat{1}{\underset{B}{B^{\prime}}}_{\overline{\hat{1}}} \\
& =\tilde{Q}_{E_{+1}}\left(\hat{x} \underset{B^{\prime}}{\otimes} \hat{y}\right)
\end{aligned}
$$

for all $x, y \in B^{\prime}$. This gives $\varphi\left(\tilde{P}_{E_{+1}}\right)=\tilde{Q}_{E_{+1}}$. On the other hand, since $\varphi\left(\tilde{P}_{E_{-1}}\right)$ and $\tilde{Q}_{E_{-1}}$ are both $B^{\prime}-B^{\prime}$ linear, in order to show their equality, it suffices to show that $\varphi\left(\tilde{P}_{E_{-1}}\right)\left(\overline{\hat{1}} \otimes_{A} \hat{1}\right)=\tilde{Q}_{E_{-1}}\left(\overline{\hat{1}} \otimes_{A} \hat{1}\right)$. Again, from 
condition $(d)$ of part $(i)$ and definition of perturbation, we have

$$
\begin{aligned}
& \varphi\left(\tilde{P}_{E_{-1}}\right)(\overline{\hat{1}} \underset{A}{\otimes} \hat{1})=\delta_{+}^{-2} \sum_{j, j^{\prime}}\left((w \underset{A}{\otimes} u) \circ\left(i d_{\mathcal{H}} \underset{B}{\otimes} \tilde{P}_{E_{-1}} \underset{A}{\otimes} i d_{\overline{\mathcal{H}}}\right)\right)\left(\eta_{j} \underset{B}{\otimes} \bar{\eta}_{j}{\underset{A}{\otimes}}_{\eta_{j^{\prime}}}{\underset{B}{B}}_{j^{\prime}}\right) \\
& =\left(\delta \delta_{+}\right)^{-1} \sum_{j, j^{\prime}, i}(w \underset{A}{\otimes} u)\left(\eta_{j} \underset{B}{\otimes}\left\langle\eta_{j}, \eta_{j^{\prime}}\right\rangle_{B} \bar{\xi}_{i} \underset{A}{\otimes} \xi_{i} \underset{B}{\otimes} \bar{\eta}_{j^{\prime}}\right) \\
& =\left(\delta \delta_{+}\right)^{-1} \sum_{j^{\prime}, i} \overline{B^{\prime}\left\langle\eta_{j^{\prime}}, \xi_{i}\right\rangle}{\underset{A}{\otimes}}_{B^{\prime}}\left\langle\eta_{j^{\prime}}, \xi_{i}\right\rangle=\left(\delta \delta_{+}\right)^{-1} \sum_{k, j^{\prime}, i} \overline{E_{A}\left(B^{\prime}\left\langle\eta_{j^{\prime}}, \xi_{i}\right\rangle c_{k}^{*}\right) \hat{c}_{k}}{\underset{A}{\otimes}}_{B^{\prime}}\left\langle\eta_{j^{\prime}}, \xi_{i}\right\rangle \\
& =\left(\delta \delta_{+}\right)^{-1} \sum_{k, j^{\prime}, i} \overline{\hat{c}}_{k}{\underset{A}{\otimes}}_{B^{\prime}}\left\langle\eta_{j^{\prime}},{ }_{A}\left\langle\xi_{i}, c_{k} \eta_{j^{\prime}}\right\rangle \xi_{i} \hat{\rangle}=\delta^{-1} \sum_{k} \overline{\hat{c}}_{k}{\underset{A}{\otimes}}_{\hat{c}_{k}} \hat{c}_{k}\right. \\
& =\tilde{Q}_{E_{-1}}\left(\overline{\hat{1}}_{A}^{\otimes} \hat{1}\right) \text {, }
\end{aligned}
$$

where $\left\{c_{k}\right\}$ is a left basis for the subfactor $A \subset B^{\prime}$. Thus, in view or Remark 2.2, $\varphi$ is an isomorphism of *-planar algebras.

We now proceed to associate a unimodular bimodule planar algebar to a finite index type $I I_{1}$ subfactor $N \subset M$. For this, the obvious thing to consider, is the unimodular bimodule planar algebra associated to ${ }_{N} L^{2}(M)_{M}$. However, we would like to find out the actions of tangles on the relative commutants (instead of intertwiner spaces) and a set of conditions which uniquely determines the action exactly the way [Jon2, Theorem 4.2.1] states for extremal subfactors. Before doing so, we first set up some notations and recall certain standard subfactor theory facts - see [Bis, Jon1, JS, PP2, PP1].

Let $N \subset M$ be a subfactor with $\delta^{2}:=[M: N]<\infty(\delta>0)$ and $\left\{M_{k}\right\}_{k \geq 1}$ be a tower of basic constructions with $\left\{e_{k} \in \mathscr{P}\left(M_{k}\right)\right\}_{k \geq 1}$ being a set of Jones projections. We will have instances to apply the following useful fact.

Lemma 5.3. PP1 For each $x_{1} \in M_{1}$, there is a unique $x \in M$ satisfying $x_{1} e_{1}=x e_{1}$; this unique element is given by $x=[M: N] E_{M}\left(x_{1} e_{1}\right)$.

For each $k \geq 1$, set $e_{[-1, k]}=\delta^{k(k+1)}\left(e_{k+1} e_{k} \cdots e_{1}\right)\left(e_{k+2} e_{k+1} \cdots e_{2}\right) \cdots\left(e_{2 k+1} e_{2 k} \cdots e_{k+1}\right) \in N^{\prime} \cap M_{2 k+1}$, $e_{[0, k]}=\delta^{k(k-1)}\left(e_{k+1} e_{k} \cdots e_{2}\right)\left(e_{k+2} e_{k+1} \cdots e_{2}\right) \cdots\left(e_{2 k} e_{2 k-1} \cdots e_{k+1}\right) \in M^{\prime} \cap M_{2 k}$ and $v_{k}=\delta^{k} e_{k} e_{k-1} \cdots e_{1} \in$ $N^{\prime} \cap M_{k}$. Then, the tower of $I I_{1}$ factors $N \subset M_{k} \subset M_{2 k+1}$ (resp., $M \subset M_{k} \subset M_{2 k}$ ) is an instance of basic construction with $e_{[-1, k]}$ (resp., $e_{[0, k]}$ ) as Jones projection, that is, there exists an isomorphism $\varphi_{-1, k}: M_{2 k+1} \longrightarrow \mathcal{L}_{N}\left(L^{2}\left(M_{k}\right)\right.$ ) (resp., $\varphi_{0, k}: M_{2 k} \longrightarrow \mathcal{L}_{M}\left(L^{2}\left(M_{k}\right)\right)$ ) given by

$$
\begin{aligned}
& \varphi_{-1, k}\left(x_{2 k+1}\right) \hat{x}_{k}=\delta^{2(k+1)} E_{M_{k}}\left(x_{2 k+1} x_{k} e_{[-1, k]}\right)^{\hat{\gamma}} \\
& \left(\text { resp., } \varphi_{0, k}\left(x_{2 k}\right) \hat{x}_{k}=\delta^{2 k} E_{M_{k}}\left(x_{2 k} x_{k} e_{[0, k]}\right)^{\hat{)}}\right)
\end{aligned}
$$

for all $x_{i} \in M_{i}, i=k, 2 k, 2 k+1$, which is identity restricted to $M_{k}$ and sends $e_{[-1, k]}$ (resp., $\left.e_{[0, k]}\right)$ to the projection with range $L^{2}(N)$ (resp., $\left.L^{2}(M)\right)$. Also, $\varphi_{-1, k}\left(M_{i}^{\prime} \cap M_{2 k+1}\right)={ }_{M_{i}} \mathcal{L}_{N}\left(L^{2}\left(M_{k}\right)\right.$ ) (resp., $\left.\varphi_{0, k}\left(M_{i}^{\prime} \cap M_{2 k}\right)={ }_{M_{i}} \mathcal{L}_{M}\left(L^{2}\left(M_{k}\right)\right)\right)$ and $\varphi_{0, k}=\left.\varphi_{-1, k}\right|_{M_{2 k}}$ for all $k \geq 0,-1 \leq i \leq k$. Further, for each $k \geq 0$, we have an $M_{k}-M$ linear unitary given (on bounded vectors) by

$$
L^{2}\left(M_{k}\right) \underset{N}{\otimes} L^{2}(M) \ni \hat{y} \underset{N}{\otimes} \hat{z} \stackrel{u_{k}}{\longmapsto}\left(y v_{k+1} z\right) \hat{)} \in L^{2}\left(M_{k+1}\right)
$$

for all $y \in M_{k}$ and $z \in M$. This unitary also satisfies the equation $\varphi_{0, k+1}(x)=u_{k}\left(\varphi_{-1, k}(x) \underset{N}{\otimes} i d_{L^{2}(M)}\right) u_{k}^{*}$ for all $x \in M_{2 k+1}$. 
Apart from these, if ${ }_{N} \mathcal{H}_{M}$ denotes the bimodule ${ }_{N} L^{2}(M)_{M}$, then for each $k \geq 1$, we have a bunch of unitary intertwiners (determined by the following actions on the bounded vectors)

$$
\begin{aligned}
& \mathcal{H}_{+2 k} \ni \hat{x}_{1} \underset{M}{\otimes} \overline{\hat{x}}_{2} \underset{N}{\otimes} \cdots \underset{M}{\otimes} \overline{\hat{x}}_{2 k} \quad \stackrel{w_{+2 k}}{\stackrel{w_{N-N}}{\longrightarrow}} \quad\left(x_{1} x_{2}^{*} v_{1} x_{3} x_{4}^{*} v_{2} x_{5} \cdots x_{2 k-2}^{*} v_{k-1} x_{2 k-1} x_{2 k}^{*}\right)^{\Upsilon} \in L^{2}\left(M_{k-1}\right), \\
& \mathcal{H}_{-2 k} \ni \overline{\hat{x}}_{1} \underset{N}{\otimes} \hat{x}_{2} \underset{M}{\otimes} \cdots \underset{N}{\otimes} \hat{x}_{2 k} \quad \stackrel{\underset{M-M}{w_{-2 k}}}{\stackrel{\leftrightarrow}{\rightleftarrows}} \quad\left(x_{1}^{*} v_{1} x_{2} x_{3}^{*} v_{2} x_{4} \cdots x_{2 k-3}^{*} v_{k-1} x_{2 k-2} x_{2 k-1}^{*} v_{k} x_{2 k}\right) \tilde{\varepsilon} \in L^{2}\left(M_{k}\right), \\
& \mathcal{H}_{+(2 k-1)} \ni \hat{x}_{1} \underset{M}{\otimes} \overline{\hat{x}}_{2} \underset{N}{\otimes} \cdots \underset{N}{\otimes} \hat{x}_{2 k-1} \underset{N-M}{\stackrel{w_{+(2 k-1)}}{\stackrel{N-M}{M}}}\left(x_{1} x_{2}^{*} v_{1} x_{3} x_{4}^{*} v_{2} x_{5} \cdots x_{2 k-2}^{*} v_{k-1} x_{2 k-1}\right) \in L^{2}\left(M_{k-1}\right), \\
& \mathcal{H}_{-(2 k-1)} \ni \overline{\hat{x}}_{1} \underset{N}{\otimes} \hat{x}_{2} \underset{M}{\otimes} \cdots \underset{M}{\otimes} \overline{\hat{x}}_{2 k-1} \underset{M-N}{\stackrel{w_{-(2 k-1)}}{\stackrel{\leftrightarrow}{\longrightarrow}}}\left(x_{1}^{*} v_{1} x_{2} x_{3}^{*} v_{2} x_{4} \cdots x_{2 k-3}^{*} v_{k-1} x_{2 k-2} x_{2 k-1}^{*}\right) \in L^{2}\left(M_{k-1}\right)
\end{aligned}
$$

and a very useful formula (see [Jon2]) $x_{1} v_{1} x_{2} v_{2} \cdots v_{k} x_{k+1}=x_{1} v_{k}^{*} x_{2} v_{k-1}^{*} \cdots v_{1}^{*} x_{k+1}$ for all $x_{i} \in M, 1 \leq i \leq 2 k$.

We are now ready to present the extension of Jones' Theorem [Jon2, Theorem 4.2.1], which associates a unimodular bimodule planar algebra to a finite index subfactor and gives a natural correspondence between extremality and sphericality.

Theorem 5.4. Let $M_{-1}:=N \subset M=: M_{0}$ be a subfactor with $\delta^{2}:=[M: N]<\infty(\delta>0)$ and $\left\{M_{k}\right\}_{k \geq 1}$ be a tower of basic constructions with $\left\{e_{k} \in \mathscr{P}\left(M_{k}\right)\right\}_{k \geq 1}$ being a set of Jones projections. Then, $P$ defined by $P_{\varepsilon k}=N^{\prime} \cap M_{k-1}$ or $M^{\prime} \cap M_{k}$ according as $\varepsilon=+$ or - , has a unique unimodular bimodule planar algebra structure with the $*$-structure given by the usual $*$ of the relative commutants such that for each $k \in \mathbb{N}_{0}$,

(1) the action of multiplication tangles is given by the usual multiplication in the relative commutants,

(2) the action of the left inclusion tangle $L I_{-k}$ is given by the usual inclusion $M^{\prime} \cap M_{k} \subset N^{\prime} \cap M_{k}$,

(3) the action of the right inclusion tangle $R I_{+k}$ is given by the usual inclusion $M_{k-1} \subset M_{k}$,

(4) $P_{E_{+(k+1)}}=\delta e_{k+1}$

(5) $P_{L E_{+(k+1)}}=\delta^{-1} \sum_{i} b_{i}^{*} x b_{i}$ for all $x \in P_{+(k+1)}$,

where $\left\{b_{i}\right\}_{i}$ is a left basis for the subfactor $N \subset M$. In particular, $P$ is spherical if and only if the subfactor $N \subset M$ is extremal. (P will be referred as the planar algebra associated to the tower $\left\{M_{k}\right\}_{k \geq-1}$ with Jones projections $\left\{e_{k}\right\}_{k \geq 1}$.)

Proof: Let $Q$ denote the bimodule planar algebra associated to the bifinite bimodule ${ }_{N} \mathcal{H}_{M}:={ }_{N} L^{2}(M)_{M}$ (as in Theorem 5.2) and $\tilde{Q}=Q^{(\delta, 1)}$ be its normalization. With notations as above, we have the *-algebra isomorphisms

$$
\begin{aligned}
& P_{+2 k}=N^{\prime} \cap M_{2 k-1} \ni x \quad \stackrel{\chi_{+2 k}}{\longrightarrow} \quad A d_{w_{+2 k}^{*}}\left(\varphi_{-1, k-1}(x)\right) \quad \in{ }_{N} \mathcal{L}_{N}\left(\mathcal{H}_{+2 k}\right)=Q_{+2 k}=\tilde{Q}_{+2 k}, \\
& P_{-2 k}=M^{\prime} \cap M_{2 k} \ni x \quad \stackrel{\chi_{-2 k}}{\longmapsto} \quad A d_{w_{-2 k}^{*}}\left(\varphi_{0, k}(x)\right) \quad \in{ }_{M} \mathcal{L}_{M}\left(\mathcal{H}_{-2 k}\right)=Q_{-2 k}=\tilde{Q}_{-2 k}, \\
& P_{+(2 k+1)}=N^{\prime} \cap M_{2 k} \ni x \stackrel{\chi_{+(2 k+1)}}{\longmapsto} A d_{w_{+(2 k+1)}^{*}}\left(\varphi_{0, k}(x)\right) \quad \in{ }_{N} \mathcal{L}_{M}\left(\mathcal{H}_{+(2 k+1)}\right)=Q_{+(2 k+1)}=\tilde{Q}_{+(2 k+1)}, \\
& P_{-(2 k+1)}=M^{\prime} \cap M_{2 k+1} \ni x \stackrel{\chi_{-(2 k+1)}}{\longmapsto} A d_{w_{-(2 k+1)}^{*}}\left(\varphi_{-1, k}(x)\right) \quad \in_{M} \mathcal{L}_{N}\left(\mathcal{H}_{-(2 k+1)}\right)=Q_{-(2 k+1)}=\tilde{Q}_{-(2 k+1)}
\end{aligned}
$$

for $k \in \mathbb{N}_{0}$. We provide $P$ with a unimodular bimodule planar algebra structure from that of $\tilde{Q}$ as follows: For each tangle $T:\left(\varepsilon_{1} k_{1} \times \cdots \times \varepsilon_{b} k_{b}\right) \rightarrow \varepsilon_{0} k_{0}$ (resp., $\left.T: \emptyset \rightarrow \varepsilon_{0} k_{0}\right)$, we define $P_{T}=\chi_{\varepsilon_{0} k_{0}}^{-1} \circ \tilde{Q}_{T} \circ\left(\times_{i=1}^{b} \chi_{\varepsilon_{i}, k_{i}}\right)$ (resp., $\left.P_{T}=\chi_{\varepsilon_{0} k_{0}}^{-1}\left(\tilde{Q}_{T}\right)\right)$. Thus, $P$ inherits a unimodular bimodule planar algebra structure with modulus $(\delta, \delta)$. We now show that $P$ satisfies all the conditions in the statement, which forces the planar algebra structure on $P$ to be unique by Remark 2.2 (2). To begin with, note that, (1) needs no further verification. We will establish the relations in (2) - (5) only for even $k$ 's because the proofs for odd $k$ 's, are completely analogous to those in the even case.

(2) Suppose $k=2 l$ and $y \in P_{-2 l}=M^{\prime} \cap M_{2 l}$. Unravelling the definitions, we just need to show that $\varphi_{0, l}(y)=A d_{w_{+(2 l+1)}}\left(i d_{\mathcal{H}} \underset{M}{\otimes} \chi_{-2 l}(y)\right)$. For $\xi=\hat{x}_{1} \underset{M}{\otimes} \overline{\hat{x}}_{2} \underset{N}{\otimes} \cdots \underset{N}{\otimes} \hat{x}_{2 l-1} \underset{M}{\otimes} \overline{\hat{x}}_{2 l} \underset{N}{\otimes} \hat{x}_{2 l+1} \in \mathcal{H}_{+(2 l+1)}^{o}$ where $x_{i} \in M$ 
for all $1 \leq i \leq 2 l+1$, we have

$$
\begin{aligned}
& w_{+(2 l+1)} \circ\left(i d_{L^{2}(M)}{\underset{M}{\otimes}}_{\chi_{-2 l}}(y)\right)(\xi) \\
& =w_{+(2 l+1)}\left(\hat{x}_{1} \underset{M}{\otimes}\left(w_{-2 l}^{*} \varphi_{0, l}(y) w_{-2 l}\right)\left(\overline{\hat{x}}_{2} \underset{N}{\otimes} \cdots \underset{N}{\otimes} \hat{x}_{2 l-1} \underset{M}{\otimes} \overline{\hat{x}}_{2 l} \underset{N}{\otimes} \hat{x}_{2 l+1}\right)\right)=\sum_{i}\left(x_{1} z_{1, i}^{*} v_{1} z_{2, i} z_{3, i}^{*} v_{2} \cdots v_{l} z_{2 l, i}\right)
\end{aligned}
$$

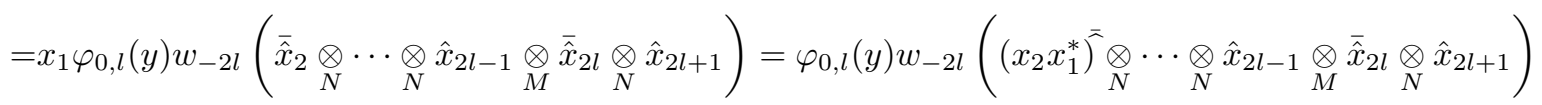

$$
\begin{aligned}
& =\varphi_{0, l}(y) w_{+(2 l+1)}(\xi) \text {, }
\end{aligned}
$$

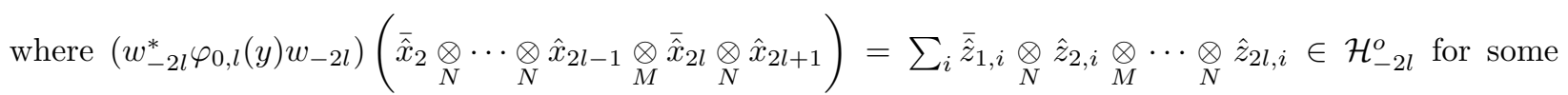
$z_{j, i} \in M$ and $\{i\}$ finite, $1 \leq j \leq 2 l$; as was desired.

(3) First consider $k=2 l, y \in P_{+2 l}=N^{\prime} \cap M_{2 l-1}$ and $\xi=\hat{x}_{1} \underset{M}{\otimes} \overline{\hat{x}}_{2} \underset{N}{\otimes} \cdots \underset{M}{\otimes} \overline{\hat{x}}_{2 l} \underset{N}{\otimes} \hat{x}_{2 l+1} \in \mathcal{H}_{+(2 l+1)}^{o}$ with $x_{i}$ 's as above, we have

$$
\begin{aligned}
& \chi_{+(2 l+1)}(y)(\xi)=\delta^{2 l} w_{+(2 l+1)}^{*}\left(E_{M_{l}}\left(y x_{1} x_{2}^{*} v_{1} \cdots x_{2 l}^{*} v_{l} x_{2 l+1} e_{[0, l]}\right)^{\uparrow}\right) \\
& =w_{+(2 l+1)}^{*} \circ \varphi_{0, l}(y) \circ u_{l-1}\left(w_{+2 l}\left(\hat{x}_{1} \underset{M}{\otimes} \overline{\hat{x}}_{2} \underset{N}{\otimes} \cdots \underset{N}{\otimes} x_{2 l-1} \underset{M}{\otimes} \overline{\hat{x}}_{2 l}\right) \underset{N}{\otimes} \hat{x}_{2 l+1}\right) \\
& =w_{+(2 l+1)}^{*} \circ u_{l-1,0} \circ\left(\varphi-1, l-1(y) \underset{N}{\otimes} i d_{L^{2}(M)}\right)\left(w_{+2 l}\left(\hat{x}_{1} \underset{M}{\otimes} \overline{\hat{x}}_{2} \underset{N}{\otimes} \cdots \underset{N}{\otimes} x_{2 l-1} \underset{M}{\otimes} \overline{\hat{x}}_{2 l}\right) \underset{N}{\otimes} \hat{x}_{2 l+1}\right)
\end{aligned}
$$

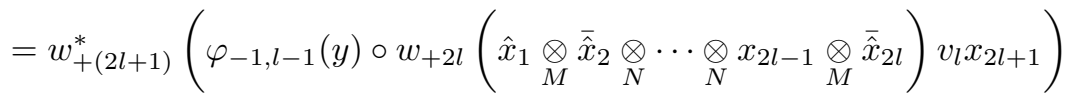

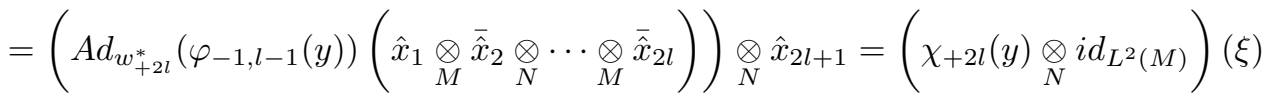

$$
\begin{aligned}
& =\tilde{Q}_{R I_{+2 l}}(\chi+2 l(y))(\xi) \text {. }
\end{aligned}
$$

On the other hand, if $k$ is odd, say $2 l+1, y \in P_{+(2 l+1)}=N^{\prime} \cap M_{2 l}$ and $\xi=\hat{x}_{1} \underset{M}{\otimes} \overline{\hat{x}}_{2} \underset{N}{\otimes} \cdots \underset{N}{\otimes} \hat{x}_{2 l+1} \underset{M}{\otimes} \overline{\hat{x}}_{2 l+2} \in$ $\mathcal{H}_{+(2 l+2)}^{o}$, then

$$
\begin{aligned}
& \tilde{Q}_{R I_{+(2 l+1)}}\left(\chi_{+(2 l+1)}(y)\right)(\xi) \\
= & \left(\chi_{+(2 l+1)}(y) \underset{M}{\otimes} i d_{\overline{\mathcal{H}}}\right)(\xi)=w_{+(2 l+1)}^{*}\left(\varphi_{0, l}(y)\left(x_{1} x_{2}^{*} v_{1} \cdots v_{l-1} x_{2 l-1} x_{2 l}^{*} v_{l} x_{2 l+1}\right)\right) \underset{M}{\otimes} \overline{\hat{x}}_{2 l+2} \\
= & w_{+(2 l+2)}^{*}\left(\varphi_{0, l}(y)\left(\left(x_{1} x_{2}^{*} v_{1} \cdots v_{l} x_{2 l+1}\right)^{\wedge}\right) x_{2 l+2}^{*}\right)=w_{+(2 l+2)}^{*}\left(\varphi_{0, l}(y)\left(x_{1} x_{2}^{*} v_{1} \cdots v_{l} x_{2 l+1} x_{2 l+2}^{*}\right)^{\wedge}\right) \\
= & w_{+(2 l+2)}^{*}\left(\varphi_{-1, l}(y)\left(x_{1} x_{2}^{*} v_{1} \cdots v_{l} x_{2 l+1} x_{2 l+2}^{*}\right)^{\hat{}}\right)=\chi_{+(2 l+2)}(y)(\xi) .
\end{aligned}
$$

(4) The initial case $k=1$ is trivial. Let $k=2 l$. For $\xi=\hat{x}_{1} \underset{M}{\otimes} \overline{\hat{x}}_{2} \underset{N}{\otimes} \cdots \underset{M}{\otimes} \overline{\hat{x}}_{2 l} \underset{N}{\otimes} \hat{x}_{2 l+1} \in \mathcal{H}_{+(2 l+1)}^{o}$ with $x_{i}$ 's in $M$, we have

$$
\begin{aligned}
\chi_{2 l+1}\left(\delta e_{2 l}\right)(\xi) & =\delta^{2 l+1} w_{2 l+1}^{*}\left(E_{M_{l}}\left(e_{2 l} x_{1} x_{2}^{*} v_{1} \cdots x_{2 l}^{*} v_{l} x_{2 l+1} e_{[0, l]}\right)^{-}\right) \\
& =\delta^{2 l+1} w_{2 l+1}^{*}\left(x_{1} x_{2}^{*} v_{1} \cdots v_{l} x_{2 l+1} E_{M_{l}}\left(e_{2 l} e_{[0, l]}\right)^{-}\right)=\delta w_{2 l+1}^{*}\left(\left(x_{1} x_{2}^{*} v_{1} \cdots v_{l} x_{2 l+1} e_{2}\right)^{\uparrow}\right) \\
& =\delta w_{2 l+1}^{*}\left(\left(x_{1} x_{2}^{*} v_{l}^{*} \cdots v_{1}^{*} x_{2 l+1} e_{2}\right)^{\kappa}\right)=w_{2 l+1}^{*}\left(\left(x_{1} x_{2}^{*} v_{l}^{*} \cdots x_{2 l-2}^{*} v_{2}^{*} x_{2 l-1} x_{2 l}^{*} x_{2 l+1}\right)^{-}\right)
\end{aligned}
$$

where, in the third equality, we have used the fact that $E_{M_{l}}\left(e_{2 l} e_{[0, l]}\right)=\delta^{-2 l} e_{2}$, which holds by the uniqueness condition in Lemma 5.3 applied to the equality $e_{2 l} e_{[0, l]}=e_{2} e_{[0, l]}$ (a routine verification involving TemperleyLieb relations satisfied by the Jones' projections $\left\{e_{l}\right\}$ ). On the other hand, by the definition of $\tilde{Q}$ and 
Theorem 5.2 (i)(d), we have

$$
\begin{aligned}
& \tilde{Q}_{E_{+2 l}}(\xi)=\delta^{-1} \sum_{i} \hat{x}_{1} \underset{M}{\otimes} \overline{\hat{x}}_{2} \underset{N}{\otimes} \cdots \underset{N}{\otimes} \hat{x}_{2 l-1} \underset{M}{\otimes}\left(b_{i} x_{2 l+1}^{*} x_{2 l}\right)_{N}^{\bar{\Sigma}} \underset{N}{\otimes} \hat{b}_{i} \\
= & \delta^{-1} w_{+(2 l+1)}^{*}\left(\left(x_{1} x_{2}^{*} v_{1} \cdots x_{2 l-2}^{*} v_{l-1} x_{2 l-1} x_{2 l}^{*} x_{2 l+1} b_{i}^{*} v_{l} b_{i}\right)^{\tau}\right)=w_{+(2 l+1)}^{*}\left(\left(x_{1} x_{2}^{*} v_{l}^{*} \cdots x_{2 l-2}^{*} v_{2}^{*} x_{2 l-1} x_{2 l}^{*} x_{2 l+1}\right)^{-}\right) .
\end{aligned}
$$

(5) Let $k=2 l, y \in P_{+(2 l+1)}=N^{\prime} \cap M_{2 l}$ and $\xi=\overline{\hat{x}}_{1} \underset{N}{\otimes} \hat{x}_{2} \underset{M}{\otimes} \cdots \underset{N}{\otimes} \hat{x}_{2 l} \in \mathcal{H}_{-2 l}^{o}$ where $x_{i} \in M$ for all $1 \leq i \leq 2 l$. Then, using the precription of the action of tangles in the planar algebra $Q$ (described in Figure 4.1, we get

$$
\begin{aligned}
& \tilde{Q}_{L E_{+(2 l+1)}}\left(\chi_{+(2 l+1)}(y)\right)(\xi)=\delta^{-1} \sum_{i}\left(e_{\mathcal{H}} \underset{M}{\otimes} i d_{\mathcal{H}}\right)\left(\overline{\hat{b}}_{i} \underset{N}{\otimes} \chi_{+(2 l+1)}(y)\left(\hat{b}_{i} \underset{M}{\otimes} \overline{\hat{x}}_{1} \underset{N}{\otimes} \cdots \underset{N}{\otimes} \hat{x}_{2 l}\right)\right) \\
& =\delta^{2 l-1} \sum_{i}\left(e_{\mathcal{H}} \underset{M}{\otimes} i d_{\mathcal{H}-2 l}\right)\left(\overline{\hat{b}}_{i} \underset{N}{\otimes} w_{+(2 l+1)}^{*}\left(E_{M_{l}}\left(b_{i} x_{1}^{*} v_{l}^{*} x_{2} \cdots x_{2 l-1}^{*} v_{1}^{*} x_{2 l} e_{[0, l]}\right)^{\hat{n}}\right)\right) \\
& =\delta^{2 l-1} \sum_{i}\left(e_{\mathcal{H}} \underset{M}{\otimes} i d_{\mathcal{H}_{-2 l}}\right)\left(w_{-(2 l+2)}^{*}\left(\left(b_{i}^{*} v_{l+1}^{*} z_{l}\right)\right)\right) \\
& =\delta^{2 l-1} \sum_{i, i^{\prime}}\left(e_{\mathcal{H}} \underset{M}{\otimes} i d_{\mathcal{H}}{ }_{-2 l}\right)\left(w_{-(2 l+2)}^{*}\left(\left(b_{i}^{*} v_{l+1}^{*} b_{i^{\prime}}^{*} v_{l}^{*} E_{M_{l-1}}\left(v_{l} b_{i^{\prime}} z_{l}\right)\right)\right)\right) \\
& =\delta^{2 l-1} \sum_{i, i^{\prime}}\left(e_{\mathcal{H}} \underset{M}{\otimes} i d_{\mathcal{H}_{-2 l}}\right)\left(\overline{\hat{b}}_{i} \underset{N}{\otimes}\left(b_{i^{\prime}}^{*}\right) \underset{M}{\otimes} \underset{\hat{1}}{\otimes} \underset{N}{\otimes} w_{+(2 l-1)}^{*}\left(\left(E_{M_{l-1}}\left(v_{l} b_{i^{\prime}} z_{l}\right)\right)^{\prime}\right)\right) \\
& =\delta^{2 l-1} \sum_{i, i^{\prime}} b_{i}^{*} b_{i^{\prime}}^{*}{\underset{\hat{1}}{\otimes}}_{N} w_{+(2 l-1)}^{*}\left(\left(E_{M_{l-1}}\left(v_{l} b_{i^{\prime}} z_{l}\right)\right)^{\hat{n}}\right)=\delta^{2 l-1} \sum_{i, i^{\prime}} w_{-2 l}^{*}\left(\left(b_{i}^{*} b_{i^{\prime}}^{*} v_{l}^{*} E_{M_{l-1}}\left(v_{l} b_{i^{\prime}} z_{l}\right)\right)\right) \\
& =\delta^{2 l-1} \sum_{i} w_{-2 l}^{*}\left(\left(b_{i}^{*} z_{l}\right)^{\hat{}}\right)=\delta^{2 l-1} \sum_{i} w_{-2 l}^{*}\left(E_{M_{l}}\left(b_{i}^{*} x b_{i} x_{1}^{*} v_{l}^{*} x_{2} \cdots x_{2 l-1}^{*} v_{1}^{*} x_{2 l} e_{[0, l]}\right)^{\hat{n}}\right)=\delta^{-1} \sum_{i} \chi_{-2 l}\left(b_{i}^{*} x b_{i}\right)(\xi) \text {, }
\end{aligned}
$$

where $z_{l}:=E_{M_{l}}\left(x b_{i} x_{1}^{*} v_{l}^{*} x_{2} \cdots x_{2 l-1}^{*} v_{1}^{*} x_{2 l} e_{[0, l]}\right)$ and in the fourth and eighth equalities we have used the fact that $\left\{v_{l} b_{i}\right\}$ is a left Pimsner-Popa basis for $M_{l-1} \subset M_{l}$ - see [JS].

Remark 5.5. Starting with an extremal subfactor, the associated planar algebra in Theorem 5.4 is indeed the same as that in [Jon2, Theorem 4.2.1] since the $\operatorname{map}_{N} \mathcal{L}\left(L^{2}\left(M_{k}\right)\right)=: N^{\prime} \ni x \longmapsto \delta^{-2} \sum_{i} b_{i}^{*} x b_{i} \in M^{\prime}:=$ ${ }_{M} \mathcal{L}\left(L^{2}\left(M_{k}\right)\right)$ is the unique $\operatorname{tr}_{N^{\prime}}$ preserving conditional expectation from $N^{\prime}$ onto $M^{\prime}$. Such extension of [Jon2, Theorem 4.2.1] was first established by Michael Burns in his thesis. However, the techniques used in $\mathrm{Bur}$ are different from our proof which is built up using graphical calculus of morphisms in a pivotal bicategory. Further, Jones and Penneys, in [JP], also obtain an extension of [Jon2, Theorem 4.2.1] in a slightly general set up.

Remark 5.6. Apart from the action of the tangles given in conditions (1) - (5), we also mention below the action of few other useful tangles.

(a) $P_{R E_{+k}}=\left.\delta E_{M_{k-2}}^{M_{k-1}}\right|_{P_{+k}}$, following from conditions (3) and (4).

(b) $P_{T R_{+k}^{r}}=\left.\delta^{k} \operatorname{tr}_{M_{k-1}}\right|_{P_{+k}}$, following from the action of right conditional expectation tangle in (a).

(c) $\delta^{-k} P_{T R_{+2 l}^{l}}$ (resp., $\delta^{-k} P_{T R_{+(2 l-1)}^{l}}$ ) is given by the trace on $P_{+2 l}=N^{\prime} \cap M_{2 l-1}$ (resp., $P_{+(2 l-1)}=$ $\left.N^{\prime} \cap M_{2 l-2}\right)$ induced by the canonical trace on ${ }_{N} \mathcal{L}\left(L^{2}\left(M_{l-1}\right)\right)$ via the map $\varphi_{-1, l-1}$ (resp., $\left.\varphi_{0, l-1}\right)$; this could be derived from the precription of the action. It also turns out that this trace on $P_{+2 l}\left(\right.$ resp., $\left.P_{+(2 l-1)}\right)$ matches with the one induced by the canonical trace on ${ }_{N} \mathcal{L}\left(L^{2}\left(M_{2 l-1}\right)\right)$ (resp., ${ }_{N} \mathcal{L}\left(L^{2}\left(M_{2 l-2}\right)\right)$ ) via the usual inclusion.

Corollary 5.7. If $P$ is the planar algebra associated to the tower $\left\{M_{k}\right\}_{k \geq-1}$ with Jones projections $\left\{e_{k}\right\}_{k \geq 1}$ (as in Theorem 5.4), then

(a) $P_{E_{-k}^{\prime}}(y)=\delta \sum_{i} b_{i}^{*} e_{1} y e_{1} b_{i}$ for all $y \in P_{-k}=M^{\prime} \cap M_{k}$, where $E_{-k}^{\prime}=L I_{+(k-1)} \circ L E_{-k}$ and $\left\{b_{i}\right\}_{i}$ is a left basis for $N \subset M$,

(b) $\lambda_{n}(P)=$ the planar algebra associated to the tower $\left\{M_{k+n}\right\}_{k \geq-1}$ with Jones projections $\left\{e_{k+n}\right\}_{k \geq 1}$. 
Proof: (a) We follow the same notations as in Theorem [5.4 and prove this only for the case $k=2 l \in$ $2 \mathbb{N}$ because the case for odd $k$ 's can be proved using similar arguments. Let $a_{\mathcal{H}}$ (resp., $e_{\overline{\mathcal{H}}}$ ) denote the isomorphism $\mathcal{H} \ni \alpha \stackrel{a_{\mathcal{H}}}{\longrightarrow} \overline{\bar{\alpha}} \in \overline{\overline{\mathcal{H}}}$ (resp., the usual evaluation map from $\overline{\overline{\mathcal{H}}} \underset{M}{\otimes} \overline{\mathcal{H}}$ to $L^{2}(N)$ ), and $\xi=\overline{\hat{x}}_{1} \underset{N}{\otimes} \hat{x}_{2} \underset{M}{\otimes}$ $\cdots \otimes \hat{x}_{2 l} \in \mathcal{H}_{-2 l}^{o}$ where $x_{i} \in M$ for all $1 \leq i \leq 2 l$. Then, using the prescription of the actions of tangles, we get

$$
\begin{aligned}
& \tilde{Q}_{E_{-2 l}^{\prime}}\left(\chi_{-2 l}(y)\right)(\xi) \\
& =\delta\left(i d_{\overline{\mathcal{H}}} \underset{N}{\otimes}\left[e_{\overline{\mathcal{H}}} \circ\left(a_{\mathcal{H}} \underset{M}{\otimes} i d_{\overline{\mathcal{H}}}\right)\right] \underset{N}{\otimes} i d_{\mathcal{H}_{+(2 l-1)}}\right)\left(\overline{\hat{x}}_{1} \underset{N}{\otimes} \hat{1}_{M} \underset{M}{\otimes}\left[w_{-2 l}^{*} \circ \varphi_{0, l}(y) \circ w_{-2 l}\left(\overline{\hat{1}}_{M} \underset{N}{\otimes} \hat{x}_{2} \underset{M}{\otimes} \cdots \underset{N}{\otimes} \hat{x}_{2 l}\right)\right]\right) \\
& =\delta \sum_{i}\left(i d_{\overline{\mathcal{H}}}^{\underset{N}{\otimes}}\left[e_{\overline{\mathcal{H}}} \circ\left(a_{\mathcal{H}} \underset{M}{\otimes} i d_{\overline{\mathcal{H}}}\right)\right] \underset{N}{\otimes} i d_{\mathcal{H}_{+(2 l-1)}}\right)\left(\overline{\hat{x}}_{1} \underset{N}{\otimes} \hat{1}_{M} \underset{M}{\otimes} w_{-2 l}^{*}\left(b_{i}^{*} v_{l}^{*} E_{M_{l-1}}\left(v_{l} b_{i} z\right)\right)^{r}\right) \\
& \text { (where } \hat{z}:=\varphi_{0, l}(y) \circ w_{-2 l}\left(\overline{\hat{1}}_{M} \underset{N}{\otimes} \hat{x}_{2} \underset{M}{\otimes} \cdots \underset{N}{\otimes} \hat{x}_{2 l}\right) \text { and since }\left\{v_{l} b_{i}\right\}_{i} \text { forms a left basis for } M_{l-1} \subset M_{l} \text { ) } \\
& =\delta \sum_{i}\left(i d_{\overline{\mathcal{H}}}^{\underset{N}{\otimes}}\left[e_{\overline{\mathcal{H}}} \circ\left(a_{\mathcal{H}} \underset{M}{\otimes} i d_{\overline{\mathcal{H}}}\right)\right] \underset{N}{\otimes} i d_{\mathcal{H}_{+(2 l-1)}}\right)\left(\overline{\hat{x}}_{1} \underset{N}{\otimes} \hat{1}_{M} \underset{M}{\otimes} \overline{\hat{b}}_{i} \underset{N}{\otimes} w_{+(2 l-1)}^{*}\left(E_{M_{l-1}}\left(v_{l} b_{i} z\right)\right)^{r}\right) \\
& =\delta \sum_{i}\left(\overline{\hat{x}}_{1} \underset{N}{\otimes} E_{N}\left(b_{i}^{*}\right) w_{+(2 l-1)}^{*}\left(E_{M_{l-1}}\left(v_{l} b_{i} z\right)\right)^{\tau}\right)=\delta \overline{\hat{x}}_{1} \underset{N}{\otimes} w_{+(2 l-1)}^{*}\left(E_{M_{l-1}}\left(v_{l} z\right) \tilde{)}=\delta w_{-2 l}^{*}\left(x_{1}^{*} v_{l}^{*} E_{M_{l-1}}\left(v_{l} z\right) \tilde{)}\right.\right. \\
& =\delta^{3} w_{-2 l}^{*}\left(x_{1}^{*} v_{l-1}^{*} e_{l} E_{M_{l-1}}\left(e_{l} v_{l-1} z\right)\right)^{\hat{n}}=\delta w_{-2 l}^{*}\left(x_{1}^{*} v_{l-1}^{*} e_{l} v_{l-1} z\right) \hat{=}=w_{-2 l}^{*}\left(x_{1}^{*} v_{1} \varphi_{0, l}(y)\left(v_{1} x_{2} x_{3}^{*} v_{2} \cdots v_{l} x_{2 l}\right) \hat{)}\right.
\end{aligned}
$$

On the other hand,

$$
\begin{aligned}
\delta \sum_{i} \chi_{-2 l}\left(b_{i}^{*} e_{1} y e_{1} b_{i}\right)(\xi) & =\delta^{2 l+1} \sum_{i} w_{-2 l}^{*}\left(E_{M_{l}}\left(b_{i}^{*} e_{1} y e_{1} b_{i} x_{1}^{*} v_{1} x_{2} x_{3}^{*} v_{2} \cdots v_{l} x_{2 l} e_{[0, l]}\right) \hat{)}\right. \\
& =\delta^{2 l+1} \sum_{i} w_{-2 l}^{*}\left(b_{i}^{*} E_{N}\left(b_{i} x_{1}^{*}\right) e_{1} E_{M_{l}}\left(y v_{1} x_{2} x_{3}^{*} v_{2} \cdots v_{l} x_{2 l} e_{[0, l]}\right) \hat{)}\right. \\
& =w_{-2 l}^{*}\left(x_{1}^{*} v_{1} \varphi_{0, l}(y)\left(v_{1} x_{2} x_{3}^{*} v_{2} \cdots v_{l} x_{2 l}\right) \hat{)} .\right.
\end{aligned}
$$

(b) Since $\lambda_{n}$ is additive with respect to $n$, it is enough to prove the statement for $n=1$. Note that $\lambda_{1}\left(I_{\varepsilon k}\right)=E_{\varepsilon(k+1)}^{\prime}$ for all colors $\varepsilon k$. By part (a) (resp., conditions (2) and (5) of Theorem 5.4), we get $\lambda_{1}(P)_{-k}=M_{1}^{\prime} \cap M_{k+1}$ (resp., $\lambda_{1}(P)_{+k}=M^{\prime} \cap M_{k}$ ). So, by the uniqueness part of Theorem 5.4 it remains to check whether the conditions (1) to (5) therein hold in this case; these verifications are completely straight forward and we will skip them.

5.2. Reconstruction of bimodule. Starting with a bimodule planar algebra, we will construct a bifinite bimodule whose associated bimodule planar algebra is isomorphic to the one which we started with. We extensively use the techniques of constructing an extremal subfactor from a spherical unimodular bimodule planar algebra in [JSW] and [KS2]; in fact, we first show that their construction with necessary modifications, works without the assumption of sphericality.

Let $P$ be a unimodular bimodule planar algebra with modulus $(\delta, \delta)$ such that $\delta>1$. We will work only with the positive part of $P$; so, for the time being, we will write $P_{k}$ in place of $P_{+k}$. Henceforth, we will exactly follow the set-up of [JSW] and [KS2 and not mention this fact at every step; whenever some modifications become necessary, we will explicitly mention them. Set $P^{k}=\underset{l \in \mathbb{N}_{0}}{\oplus} P_{l}^{k}$ where $P_{l}^{k}:=P_{k+l}$ for $k, l \in \mathbb{N}_{0}$. Define the following structures on $P^{k}$ :

(1) Multiplication:

$$
P^{k} \times P^{k} \supset P_{l}^{k} \times P_{m}^{k} \ni(x, y) \mapsto x \cdot y:=\left.\sum_{i=0}^{2(l \wedge m)} P \underbrace{k}_{+\mid 2 l-i} \underbrace{k}_{i}\right|_{i=0} ^{k} P_{l+m-i}^{k} \subset P^{k}
$$

We will also denote the element in the sum when $i=0$, by $x \odot y$; the $i$-th element will be denoted by $x \underset{i}{\odot} y$. 
(2) *-structure:

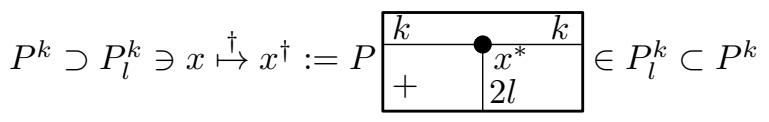

(3) Trace:

$$
P^{k} \supset P_{l}^{k} \ni x \stackrel{t_{k}}{\mapsto} t_{k}(x):=\delta_{l=0} \delta^{-k} P_{T R_{k}^{l}}(x) \in \mathbb{C}
$$

$P^{k}$ becomes an associative $*$-algebra with respect to (1) and (2).

Remark 5.8. The trace-functional on $P^{k}$ which was defined in [JSW] KS2], does not satisfy tracial property in the absence of sphericality. So, we had to take this specific spherical isotopy of the trace in [JSW, KS2] so that the functional defined in (3), is indeed a trace on $P^{k}$.

To see the tracial property, note that for $x \in P_{l}^{k}, y \in P_{m}^{k}$,

$$
t_{k}(x \cdot y)=\delta_{l=m} \delta^{-k} P\left(\begin{array}{l}
x^{0} \\
2 l \\
y
\end{array}\right)=t_{k}(y \cdot x) .
$$

We define a sesquilinear form $\langle\cdot, \cdot\rangle$ on $P^{k}$ by $\langle x, y\rangle=t_{k}\left(x^{\dagger} \cdot y\right)$ for $x, y \in P^{k}$. It is straight forward to check that $(i) t_{k}$ is positive definite and hence the sesquilinear form gives an inner product, $(i i)\left\{P_{l}^{k}\right\}_{l \in \mathbb{N}_{0}}$ are mutually orthogonal subspaces of $P^{k}$ with respect to this inner product. Let $L: P^{k} \rightarrow \operatorname{End}_{\mathbb{C}}\left(P^{k}\right)$ and $R: P^{k} \rightarrow \operatorname{End}_{\mathbb{C}}\left(P^{k}\right)$ denote the left and right multiplication operators respectively. The proof of the boundedness of these operators in [JSW, KS2] needs a little modification in the non-spherical case because the inner product is slightly different; however, the main idea of the proof will remain the same.

Lemma 5.9. $L_{a}$ and $R_{a}$ are bounded for $a \in P^{k}$.

Proof: Without loss of generality, let $a \in P_{l}^{k}$ for $l \in \mathbb{N}_{0}$. Now, $L_{a}=\sum_{i=0}^{2 l} L_{a}^{i}$ where $L_{a}^{i}: P^{k} \rightarrow P^{k}$ is defined by

$$
P^{k} \supset P_{m}^{k} \ni x \stackrel{L_{a}^{i}}{\longmapsto} \delta_{i \leq 2 m}(\underset{i}{a} x) \in P_{l+m-i}^{k} \subset P^{k} .
$$

Since $\left\{P_{l}^{k}\right\}_{l \in \mathbb{N}_{0}}$ are mutually orthogonal, therefore it is enough to show that there exists an $M>0$ such that $L_{a}^{i}(x) \leq M\|x\|$ for all $x \in P_{m}^{k}, m \in \mathbb{N}_{0}$. Without loss of generality, let $m>2 l$. Note that

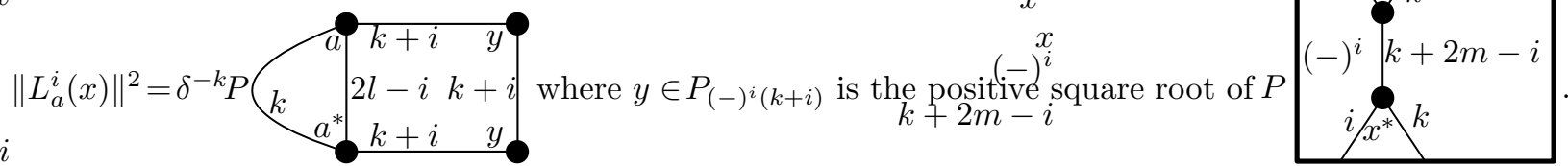

To see that this is indeed a positive element, one needs to take an appropriate rotation of $x$ and use positivity of the action of the right conditional expectation tangle from $(-)^{i}(k+m)$ to $(-)^{i}(k+i)$. Thus, $\left\|L_{a}^{i}(x)\right\|^{2}$

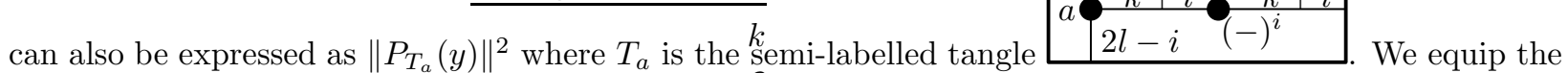
domain $P_{(-)^{i}(k+i)}$ of $P_{T_{a}}$, with the norm $\|\cdot\|^{\prime}$ coming from $\delta^{-k} P_{T R_{(-)^{i}(k+i)}^{l}}$ so that $\|y\|^{\prime}=\|x\|$. Hence, the desired inequality is satisfied by setting $M:=$ the operator norm of $P_{T_{a}}$ which is independent of $m>2 m$ and $x \in P_{m}^{k}$. Boundedness of $R_{a}$ will follow using the same kind of arguments.

Let $\mathcal{H}_{k}$ be the completion of $P^{k}$ and $M_{k}:=\left(L P^{k}\right)^{\prime \prime} \subset \mathcal{L}\left(\mathcal{H}_{k}\right)$. Note that $\hat{1}_{P^{k}} \in \mathcal{H}_{k}$ is a cyclic, separating, trace vector for $M_{k}$. The unital *-algebra inclusion $\quad P^{k} \supset P_{l}^{k} \ni x \mapsto P \overrightarrow{\begin{array}{c}k \\ +2 l \\ +2\end{array}} \in P_{l}^{k+1} \subset P^{k+1}$ induces an an inclusion $M_{k} \hookrightarrow M_{k+1}$. Note that $L P_{0}^{k} \subset M_{0}^{\prime} \cap M_{k}$. One can also prove that $P_{k} \ni x \mapsto L_{x} \in M_{0}^{\prime} \cap M_{k}$ is a $C^{*}$-algebra isomorphism and $M_{k}$ 's are $I I_{1}$-factors; for proofs, see [JSW, KS2] where $\delta>1$ is crucially used. 
Remark 5.10. The above mentioned isomorphism takes the normalized left picture trace on $P_{k}$ to the $M_{k}$ trace on $M_{0}^{\prime} \cap M_{k}$. So, even if we assume that $\left\{M_{k}\right\}_{k \geq 2}$ is a tower of basic constructions of $M_{0} \subset M_{1}$, there is no hope of the unimodular bimodule planar algebra associated to $M_{0} \subset M_{1}$ being isomorphic to $P$ (unless $P$ is spherical) because of the condition in Remark 5.6(c). We will fix this issue in the following theorem using a certain perturbation.

If $Q$ is a unimodular bimodule planar algebra, then there exists a unique invertible positive central element $z \in Q_{+1}$ such that $Q_{T R_{+1}^{l}}(\cdot)=Q_{T R_{+1}^{r}}(\cdot z)$. It is easy to check that $z$ is a weight of $Q$ and $Q_{T R_{\varepsilon k}^{l}}(\cdot)=Q_{T R_{\varepsilon k}^{r}}\left(\cdot z_{\varepsilon k}\right)$. We will refer this as the trace intertwiner weight of $Q$.

Theorem 5.11. If $Q$ is a unimodular bimodule planar algebra with modulus $(\delta, \delta)$, then there exists a finite index subfactor $M_{0} \subset M_{1}$ of type $I I_{1}$ whose associated planar algebra is isomorphic to $Q$.

Proof: Without loss of generality, we may assume $\delta>1$ because index of a bimodule planar algebra is at least 1 (which follows from positivity of the action of the trace tangles) and the index 1 case is a triviality. Let $P$ be the perturbation of $Q$ by the decomposition $z=z^{1 / 2} \cdot z^{1 / 2}$ of the trace intertwiner weight $z$ of $Q$. Clearly, $P$ is a unimodular bimodule planar algebra. Consider the tower of $I I_{1}$-factors $\left\{M_{k}\right\}_{k \geq 0}$ constructed from $P$ right before Remark 5.10 and the isomorphism $Q_{k}=P_{k} \ni x \stackrel{\phi}{\mapsto} L_{x} \in M_{0}^{\prime} \cap M_{k}$. Note that the map $Q_{R I_{k}}=P_{R I_{k}}$ under $\phi$, is given by the inclusion $M_{k} \subset M_{k+1}$. So, by Theorem 5.4 it remains to show that (i) $\left\{M_{k}\right\}_{k \geq 2}$ is a tower of basic construction of $M_{0} \subset M_{1}$ with Jones projections $\left\{e_{k}:=\delta^{-1} \phi\left(Q_{E_{+k}}\right)\right\}_{k \geq 1}$ and $(i i) \phi \circ Q_{E_{+k}^{\prime}}=\delta^{-1} \sum_{b \in B} b \phi(\cdot) b^{*}$ for all $k \geq 1$, where $B$ is a right Pimsner-Popa basis for $M_{0} \subset M_{1}$.

Proof of (i): First note that the unique trace preserving conditional expectation from $M_{k}$ to $M_{k-1}$ is induced

(via $L$ ) by the map $P^{k} \supset P_{l}^{k} \ni x \stackrel{E_{k-1}^{k}}{\longrightarrow}\left\{\begin{array}{l}\delta^{-1} P \\ \delta^{-1} P\end{array}\right.$

Using the definition of $P=Q^{\left(z^{1 / 2}, z^{1 / 2}\right)}$, it immediately follows that $Q_{E_{+k}} x Q_{E_{+k}}=\delta E_{k-1}^{k}(x) Q_{E_{+k}}$ for all $x \in P^{k}$. Thus, for all $y \in M_{k}, e_{k} y e_{k}=E_{M_{k-1}}^{M_{k}}(y) e_{k}$. Moreover, it is straight forward to check that for all $x \in P^{k+1}, Q_{E_{+k}} x=\delta Q_{E_{+k}} E_{k-1}^{k}\left(Q_{E_{+k}} x\right)$ which implies $e_{k} M_{k+1}=e_{k} M_{k}$. Thus, by Proposition 2.11, $M_{k+1}$ is a basic construction of $M_{k-1} \subset M_{k}$ with Jones projection $e_{k}$ and $\left[M_{k}: M_{k-1}\right]=\delta^{2}$.

Proof of (ii): Since $L^{2}\left(M_{1}\right) \cong \mathcal{H}_{1} \cong \underset{k \in \mathbb{N}_{0}}{\oplus} P_{k}^{1}$, therefore for each $b \in B$ and $k \in \mathbb{N}_{0}$, there exists $b_{k} \in P_{k}^{1}$ such that $\hat{b}=\sum_{k \in \mathbb{N}_{0}} \hat{L}_{b_{k}}$. Also, $\hat{b^{*}}=\sum_{k \in \mathbb{N}_{0}} \hat{L}_{b_{k}^{\dagger}}$. Since $B$ is a basis for $M_{0} \subset M_{1}$, we have

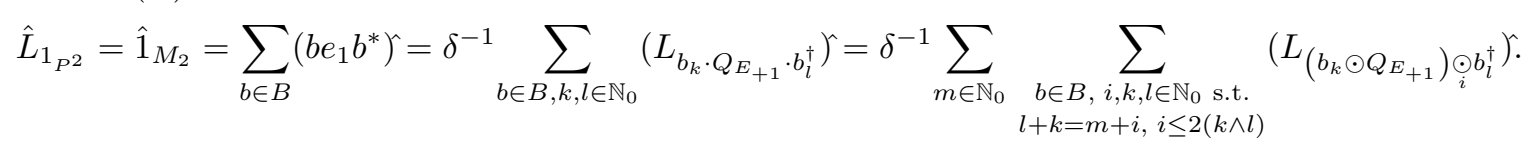

Using the relation $Q_{E_{+1}}=P_{E_{+1}^{\left(z^{-1 / 2}, z^{-1 / 2}\right)}}$, the above gives the following formula in terms of pictures:

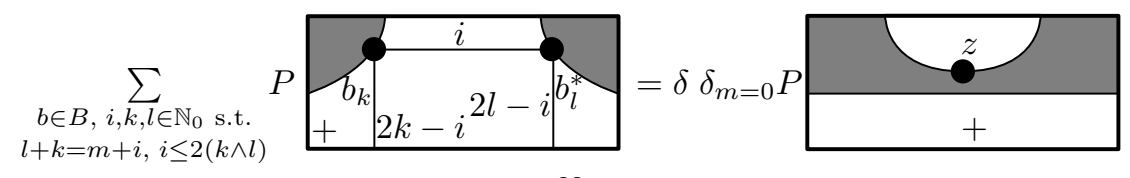


for all $m \in \mathbb{N}_{0}$ where the sum in the left hand side is with respect to the Hilbert space norm in $P^{2}$. Now, let $L_{x} \in M_{0}^{\prime} \cap M_{k}=L P_{0}^{k}$ for $x \in P_{0}^{k}=Q_{0}^{k}$. So, $\sum_{b \in B}\left(b L_{x} b^{*}\right)^{\gamma}=\sum_{m \in \mathbb{N}_{0}} \quad \sum_{b \in B, i, k, l \in \mathbb{N}_{0} \text { s.t. }}\left(L_{\left(b_{k} \odot x\right) \odot b_{i}^{\dagger}}\right)^{x}$. Note that $l+k=m+i, i \leq 2(k \wedge l)$

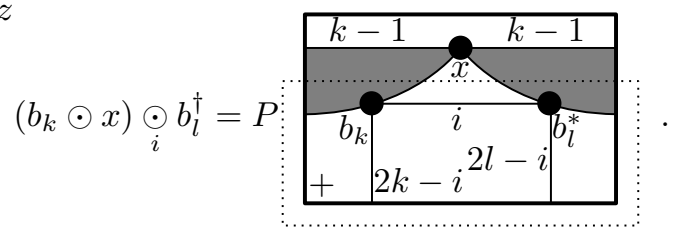

Using the isomrphism $\mathcal{H}_{k} \supset\left(P^{k}\right)^{\wedge} \ni \hat{x} \mapsto \hat{L}_{x} \in\left(M_{k}\right)^{\wedge} \subset L^{2}\left(M_{k}\right)$ and replacing the sum (in the expansion of $\left.\sum_{b \in B}\left(b L_{x} b^{*}\right)^{\prime}\right)$ of the stuff inside the dotted box by the right side of the above formula, we obtain

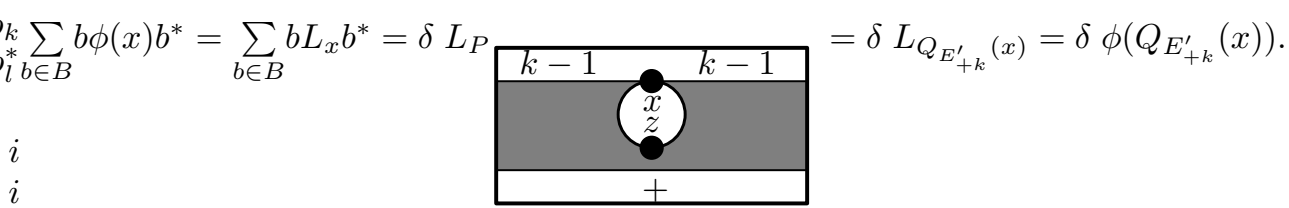

Remark 5.12. We must mention here that Popa (in Pop5]) started with his set up of 'generalized $\lambda$-lattice' (which, we believe, is analogous to a unimodular bimodule planar algebra in our case) and gave a general method of constructing a (non-extremal) subfactor whose standard invariant corresponds to the generalized $\lambda$-lattice.

Using Theorem 5.11, we now construct a bimodule from a bimodule planar algebra in the next theorem.

Theorem 5.13. Let $P$ be a bimodule planar algebra with modulus $\left(\delta_{-}, \delta_{+}\right)$such that $\left(\delta_{+} \delta_{-}\right)>1$. Then there exists a bifinite bimodule whose associated bimodule planar algebra is isomorphic to $P$.

Proof: Let $Q$ be the normalization of $P$ and $\delta:=\sqrt{\delta_{+} \delta_{-}}$. By Theorem 5.11, there exist a subfactor $N \subset M$ of type $I I_{1}$, whose associated planar algebra is isomorphic to $Q$. Without loss of generality, let $\delta_{+}>1$. Choose $p \in \mathscr{P}(M)$ such that $\operatorname{tr}_{M}(p)=\delta_{+}^{-1}$. Set $p^{\prime}:=J p J \in M^{\prime}:={ }_{M} \mathcal{L}\left(L^{2}(M)\right), \mathcal{H}:=L^{2}(M) p=$ Range $\left(p^{\prime}\right), A:=$ $N p^{\prime}$ and $B:=M_{p}$ where $J$ is the canonical anti-unitary involution on $L^{2}(M)$. Consider the bimodule ${ }_{A} \mathcal{H}_{B}$. Note that (i) $\operatorname{dim}\left({ }_{A} \mathcal{H}\right)=t r_{M^{\prime}}\left(p^{\prime}\right) \operatorname{dim}\left({ }_{N} L^{2}(M)\right)=\delta_{+}^{-1} \delta^{2}=\delta_{-},\left(\right.$ii) $\operatorname{dim}\left(\mathcal{H}_{B}\right)=\left(\operatorname{tr}_{M}(p)\right)^{-1} \operatorname{dim}\left(L^{2}(M)_{M}\right)=$ $\delta_{+}$and (iii) $B^{\prime}:=\mathcal{L}_{B}(\mathcal{H})=M p^{\prime}$. Thus, the subfactors $A \subset B^{\prime}$ and $N \subset M$ are isomorphic, and so are their associated planar algebras. The proof of Theorem 5.11 implies that $Q(=$ normalized $P$ ) is isomorphic to the normalized bimodule planar algebra associated to the bimodule ${ }_{A} L^{2}\left(B^{\prime}\right)_{B^{\prime}}$ which, by Theorem 5.2 (iii), is isomorphic to that of ${ }_{A} \mathcal{H}_{B}$. So, $P$ is isomorphic to the bimodule planar algebra associated to ${ }_{A} \mathcal{H}_{B}$.

Remark 5.14. Since we have an extension of Jones' theorem [Jon2, Theorem 4.2.1] as well as its converse for any finite index subfactor, we could have very well referred a unimodular bimodule planar algebra as a 'subfactor-planar algebra', but we abstain from doing so as the term subfactor planar algebra has already been in use for a spherical unimodular bimodule planar algebra.

\section{Examples of PeRturbations}

In this section, we will provide two examples of perturbations of bimodule planar algebras by non-scalar weights. The first holds for any non-spherical planar algebras whereas the second example is a more concrete one which involves diagonal subfactors.

6.1. Non-spherical to spherical. In Section 3, we saw that the perturbation class of every bimodule planar algebra contains a unimodular one, namely, its normalization. In view of this, we analyze the natural question whether there is more than one unimodular bimodule planar algebra (upto isomorphism) in the perturbation class of a bimodule planar algebra. The answer is negative for irreducible ones because the weights have to be scalars. 
Let $P$ be a unimodular bimodule planar algebra (not necessarily spherical) with modulus $(\delta, \delta)$. Suppose $z \in P_{+1}$ denotes the trace intertwiner weight of $P$ (discussed in Section 5.2). Note that $z^{1 / 2}$ is also a weight of $P$. Consider the planar algebra $Q:=P^{\left(z^{1 / 4}, z^{1 / 4}\right)}$. It is easy to check that $Q$ is also a bimodule planar algebra. Now, for $x \in Q_{+1}=P_{+1}$, we have

$$
Q_{T R_{+1}^{l}}(x)=P_{T R_{+1}^{l}\left(z^{1 / 4}, z^{1 / 4}\right)}(x)=P_{T R_{+1}^{l}}\left(z^{-1 / 2} x\right)=P_{T R_{+1}^{r}}\left(z^{1 / 2} x\right)=P_{T R_{+1}^{r}\left(z^{1 / 4}, z^{1 / 4}\right)}(x)=Q_{T R_{+1}^{r}}(x),
$$

where the third equality follows from the trace intertwining property of $z$ and the rest follow directly from the definition of perturbation. Setting $x=1_{P_{+1}}$ in the above equation, we conclude that $Q$ is unimodular with modulus $P_{T R_{+1}^{l}}\left(z^{-1 / 2}\right)=P_{T R_{+1}^{r}}\left(z^{1 / 2}\right)$ and thereby, is the same as its normalization; thus, $Q$ is spherical. We include this observation in the following proposition.

Proposition 6.1. The perturbation class of every bimodule planar algebra contains a unique spherical unimodular bimodule planar algebra which can also be characterized by the unimodular one having the minimal index value. In other words, any bimodule planar algebra assuming the minimal index in its perturbation class must be spherical.

Proof: To show minimality of the index, consider a spherical unimodular bimodule planar algebra $P$ with modulus $\delta$ and a positive weight $z$ of $P$. Set $Q:=P^{\left(z^{1 / 2}, z^{1 / 2}\right)}$. Let $\left\{p_{i}\right\}_{i=1}^{n}$ be the set of minimal central projections of $P_{+1}=Q_{+1}$. Note that $\delta=\sum_{i} \omega_{i}$ where $\omega_{i}=P_{T R_{+1}^{r}}\left(p_{i}\right)=P_{T R_{+1}^{l}}\left(p_{i}\right)$ for all $i$. Also, for all $i$, there exists $\lambda_{i}>0$ such that $z=\sum_{i} \lambda_{i} p_{i}$. If $\left(\delta_{-}, \delta_{+}\right)$denotes the modulus of $Q$, then $\delta_{ \pm}=\sum_{i} \lambda_{i}^{ \pm 1} \omega_{i}$. Thus,

$$
\begin{aligned}
& \operatorname{index}(Q) \\
&=\sum_{i, j=1}^{n} \frac{\lambda_{i}}{\lambda_{j}} \omega_{i} \omega_{j}=\left(\sum_{i=1}^{n} \omega_{i}^{2}\right)+\sum_{1 \leq j<k \leq n}\left(\frac{\lambda_{j}}{\lambda_{k}}+\frac{\lambda_{k}}{\lambda_{j}}\right) \omega_{i} \omega_{j} \geq\left(\sum_{i}^{n} \omega_{i}^{2}\right)+\sum_{1 \leq j<k \leq n} 2 \omega_{i} \omega_{j}=\left(\sum_{i} \omega_{i}\right)^{2} \\
&=\operatorname{index}(P)
\end{aligned}
$$

where the equality occurs if and only if $\lambda_{i}=\lambda_{j}$ for $1 \leq i, j \leq n$, that is, $z$ is a scalar weight. Now, if $\tilde{P}$ is any unimodular bimodule planar algebra assuming minimal index in its perturbation class, then there exists a weight $w$ such that $\tilde{Q}:=\tilde{P}^{\left(w^{1 / 2}, w^{1 / 2}\right)}$ is unimodular spherical. Again, by the above argument, index $(\tilde{Q})$ being minimal, is same as $\operatorname{index}(\tilde{P})$ and $w$ is a scalar weight. Since both $\tilde{P}$ and $\tilde{Q}$ are unimodular, therefore $w=1_{P_{+1}}$ and hence, $\tilde{P}=\tilde{Q}$ is spherical. This also shows the uniqueness of a spherical unimodular bimodule planar algebra in the perturbation class of a bimodule planar algebra.

Remark 6.2. Instance of minimizing index of a conditional expectation onto a subfactor already appeared in the work of Hiai (see [Hia]) and then Popa (see [Pop3]). Proposition 6.1 gives a nice way of minimizing index using perturbation of planar algebra.

Remark 6.3. From the proof of Proposition 6.1, it is clear that if the perturbation of a spherical bimodule planar algebra by a positive weight, is spherical, then the weight must be a scalar one.

Remark 6.4. It is easy to check that a finite depth bimodule planar algebra is always spherical because by Perron-Frobenius theorem, the index must be equal to the norm-square of the pricipal graph and perturbation does not change the principal graphs.

6.2. Spherical to non-spherical. Here we try to find whether we can perturb one of the known spherical unimodular planar algebras and get a non-spherical one. For this, we study the case of diagonal subfactors.

In Section 6.1. we found that the perturbation of a bimodule planar algebra $P$ by a weight $z$, has modulus $\left(P_{T R_{+1}^{l}}\left(z^{-1 / 2}\right), P_{T R_{+1}^{r}}\left(z^{1 / 2}\right)\right)$; so, for the perturbation to be unimodular, we need to find $z$ satisfying $P_{T R_{+1}^{l}}\left(z^{-1 / 2}\right)=P_{T R_{+1}^{r}}\left(z^{1 / 2}\right)$. Consider the diagonal planar algebra $P$ constructed in BDG2 with respect to the free group $\mathbb{F}_{2}$ generated by two free generators $a_{\eta}$ for $\eta \in I:=\{-,+\}$ and the trivial cocycle. We briefly recall (from [BDG2]) few aspects of this planar algebra $P$ which will be needed for further analysis.

Vector spaces: $P_{\eta 0}:=\mathbb{C}$ and $P_{\eta k}=\mathbb{C}\left\{\underline{\varepsilon} \in I^{2 k}:\right.$ alt $\left.t_{\eta}(\underline{\varepsilon})=e\right\}$ for all $k \geq 1$, where $e$ denotes the identity of $\mathbb{F}_{2}$ and alt $_{\eta}$ is given by $I^{n} \ni\left(\varepsilon_{1}, \cdots, \varepsilon_{n}\right) \stackrel{\text { alt }}{\longmapsto} \begin{cases}a_{\varepsilon_{1}}^{-1} a_{\varepsilon_{2}} a_{\varepsilon_{3}}^{-1} \cdots a_{\varepsilon_{n}}^{(-1)^{n}} \in \mathbb{F}_{2} & \text { if } \eta=+, \\ a_{\varepsilon_{1}} a_{\varepsilon_{2}}^{-1} a_{\varepsilon_{3}} \cdots a_{\varepsilon_{n}}^{(-1)^{n-1}} \in \mathbb{F}_{2} & \text { if } \eta=-.\end{cases}$ 
Action of tangles: For a tangle $T:\left(\eta_{1} k_{1}, \cdots, \eta_{b} k_{b}\right) \rightarrow \eta_{0} k_{0}$, the action $P_{T}: P_{\eta_{1} k_{1}} \times \cdots \times P_{\eta_{b} k_{b}} \rightarrow P_{\eta_{0} k_{0}}$ is given by

$$
\left\langle P_{T}\left(\underline{\varepsilon}^{1}, \cdots, \underline{\varepsilon}^{b}\right), \underline{\varepsilon}^{0}\right\rangle:=\#\left\{f:\{\text { strings in } T\} \rightarrow I|f|_{\text {marked points of } i \text {-th } \operatorname{disc} \text { in } T}=\underline{\varepsilon}^{i} \text { for } 0 \leq i \leq b\right\}
$$

where $\underline{\varepsilon}^{i}$ belongs to the distinguished basis of $P_{\eta_{i} k_{i}}$ for $0 \leq i \leq b$.

Fix a positive scalar $\lambda_{-}=\lambda_{+}^{-1}$. Consider $z:=\left[\lambda_{-}(-,-)+\lambda_{+}(+,+)\right] \in P_{+1}$ and $\lambda_{\eta \underline{\varepsilon}}:=\prod_{i=1}^{k} \lambda_{\varepsilon_{i}}^{\eta(-1)^{i-1}}$ for $\underline{\varepsilon}=\left(\varepsilon_{1}, \cdots, \varepsilon_{k}\right) \in I^{k}$. Using the relation $z^{-1}=\left[\lambda_{-}^{-1}(-,-)+\lambda_{+}^{-1}(+,+)\right] \in P_{+1}$ and the action of tangles in Definition 3.1. one can derive that $z_{\eta k}=\sum_{\underline{\varepsilon} \in I^{k}} \lambda_{\eta \underline{\varepsilon}}(\underline{\varepsilon}, \underline{\tilde{\varepsilon}}) \in P_{\eta k}$, where $\underline{\tilde{\varepsilon}}$ is the sequence obtained by reversing the order in $\underline{\varepsilon}$. From the action of the multiplication tangle $M_{\eta k}$, we have $z_{\eta k}(\underline{\varepsilon}, \underline{\tilde{\nu}}) z_{\eta k}^{-1}=$ $\lambda_{\eta \underline{\varepsilon}} \lambda_{\eta \underline{\nu}}^{-1}(\underline{\varepsilon}, \underline{\tilde{\nu}})=\lambda_{\eta(\underline{\varepsilon}, \underline{\tilde{\nu}})}(\underline{\varepsilon}, \underline{\tilde{\nu}})$ for $\underline{\varepsilon}, \underline{\nu} \in I^{k}$ such that $a l t_{\eta}(\underline{\varepsilon}, \underline{\tilde{\nu}})=e$. Freeness of $a_{-}$and $a_{+}$implies that there exists a configuration of non-crossing pairings of matching signs (abbreviated as 'NC-pairing') in the sequence $(\underline{\varepsilon}, \underline{\tilde{\nu}})$, which implies that $\lambda_{\eta(\underline{\varepsilon}, \underline{\tilde{\nu}})}=1$. Thus, $z_{\eta k}$ is central and hence, $z$ is a weight of $P$. Further, positivity of $z$ implies that $Q:=P^{\left(z^{1 / 2}, z^{1 / 2}\right)}$ is a bimodule planar algebra. To check unimodularity of $Q$, note that $P_{T R_{+1}^{l}}\left(z^{-1}\right)=\left(\lambda_{-}\right)^{-1}+\left(\lambda_{+}\right)^{-1}=\lambda_{-}+\lambda_{+}=P_{T R_{+1}^{r}}(z)$. Now, $Q$ is spherical if and only if, for all $s, t \in \mathbb{C}$,

$$
s \lambda_{-}^{-1}+t \lambda_{+}^{-1}=Q_{T R_{+1}^{l}}(s(-,-)+t(+,+))=Q_{T R_{+1}^{r}}(s(-,-)+t(+,+))=s \lambda_{-}+t \lambda_{+}
$$

if and only if $\lambda_{-}=1=\lambda_{+}$, that is, $z=1_{P_{+1}}$. Clearly, the range of the index values of these perturbed planar algebras is $[4, \infty)$. We gather the above observation in the following proposition.

Proposition 6.5. Let $P$ denote the diagonal planar algebra constructed in [BDG2, §3] with respect to the free group $\mathbb{F}_{2}$ generated by two free generators and the trivial cocycle. Then one can perturb $P$ to obtain a continuum of unimodular bimodule planar algebras $\left\{Q^{\gamma}\right\}_{\gamma \geq 4}$ such that $Q^{\gamma}$ has (i) index $\gamma$, (ii) is equal to $P$ if $\gamma=4$ and (iii) is non-spherical if and only if $\gamma \neq 4$.

Remark 6.6. These perturbed planar algebras turn out to be the ones associated to the subfactors with index greater than 4 constructed in [Jon1] (which are all non-extremal), as was pointed out by Vaughan Jones.

We give an explicit proof of the above remark in the following proposition.

Proposition 6.7. Let $N \subset M$ be a subfactor of type $I I_{1}$ with a partition of unity $\left\{p_{\varepsilon} \in \mathscr{P}\left(N^{\prime} \cap M\right) \mid \varepsilon \in I\right\}$ satisfying (i) $N p_{\varepsilon}=M_{p_{\varepsilon}}$ and (ii) $c_{-}:=\operatorname{tr}_{M}\left(p_{-}\right) \neq \operatorname{tr}_{M}\left(p_{+}\right)=: c_{+}$. (Such subfactors were constructed by Jones in [Jon1] with $M$ having full fundamental group.) Then, the planar algebra associated to $N \subset M$ is isomorphic to $Q^{\left(c_{-} c_{+}\right)^{-1}}$ (as in Proposition [6.5).

Proof: Condtions $(i)$ and $($ ii $)$ imply $p_{\varepsilon} \in \mathscr{P}_{\text {minl }}\left(N^{\prime} \cap M\right), N^{\prime} \cap M \cong \mathbb{C} p_{-} \oplus \mathbb{C} p_{+}$and $c_{\varepsilon} t r_{N^{\prime}}\left(p_{\varepsilon}\right)[M$ : $N]=\left[M_{p_{\varepsilon}}: N p_{\varepsilon}\right]=1$. So, $[M: N]=c_{-}^{-1}+c_{+}^{-1}=\left(c_{-} c_{+}\right)^{-1}$ and $\operatorname{tr}_{N^{\prime}}\left(p_{\varepsilon}\right)=c_{-\varepsilon}$ and hence, $N \subset M$ is non-extremal.

Suppose $\left\{M_{k}\right\}_{k \geq 1}$ is a tower of basic constructions of $N \subset M$ with Jones projections $\left\{e_{k}\right\}_{k \geq 1}$ and $\tilde{P}$ be

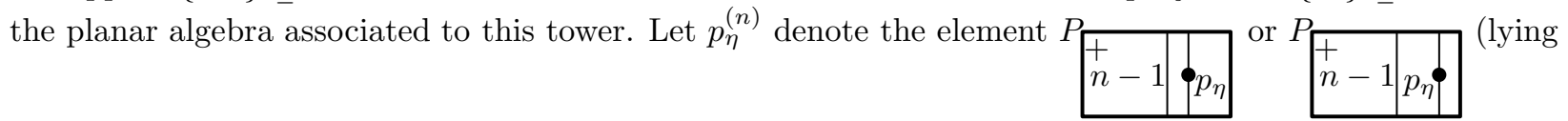
in $\mathscr{P}\left(M_{n-2}^{\prime} \cap M_{n-1}\right)$ by Corollary $\left.5.7(\mathrm{~b})\right)$ according as $n$ is even or odd, $c_{\eta}^{(n)}:=\operatorname{tr}_{M_{n-1}}\left(p_{\eta}^{(n)}\right)=c_{(-)^{n-1} \eta}$, $p_{\underline{\varepsilon}}:=p_{\varepsilon_{1}}^{(1)} \cdots p_{\varepsilon_{n}}^{(n)} \in \mathscr{P}\left(N^{\prime} \cap M_{n-1}\right)$ and $c_{\underline{\varepsilon}}:=\prod_{i=1}^{n} c_{\varepsilon_{i}}^{(i)}=\operatorname{tr}_{M_{n-1}}\left(p_{\underline{\varepsilon}}\right)$ for $\eta \in I, \underline{\varepsilon}=\left(\varepsilon_{1}, \cdots, \varepsilon_{n}\right) \in I^{n}$. Clearly, $\left\{p_{\underline{\varepsilon}}: \underline{\varepsilon} \in I^{n}\right\}$ forms a partition of unity. Further, the formula involving indices of cut-down subfactors implies $N \underline{p}_{\underline{\varepsilon}}=\left(M_{n-1}\right)_{p_{\underline{\varepsilon}}}$ and hence $p_{\underline{\varepsilon}}$ is minimal in $\tilde{P}_{n}$. The set of $t r_{M_{n-1}}$-values of these minimal projections is given by $\left\{c(n, k):=c_{-}^{k} c_{+}^{n-k} \mid 0 \leq k \leq n\right\}$.

In order to establish an isomorphism between $\tilde{P}$ and $Q^{\left(c_{-} c_{+}\right)^{-1}}$, we first prove that for $\underline{\varepsilon}, \underline{\eta} \in I^{n}, c_{\underline{\varepsilon}}=c_{\underline{\eta}}$ if and only if there exists an NC-pairing for the sequence $(\underline{\varepsilon}, \underline{\tilde{\eta}})$.

'if' part: The pairings give the equation $c_{(\underline{\varepsilon}, \underline{\tilde{\eta}})}=\left(c_{-} c_{+}\right)^{n}$. On the other hand, $c_{(\underline{\varepsilon}, \underline{\tilde{\eta}})}=c_{\underline{\varepsilon}} c_{-\underline{\eta}}=c_{\underline{\varepsilon}}\left[\frac{\left(c_{-} c_{+}\right)^{n}}{c_{\underline{\eta}}}\right]$ where $-\left(\eta_{1}, \ldots, \eta_{n}\right):=\left(-\eta_{1}, \ldots,-\eta_{n}\right)$. 
'only if' part: We use induction on the length $n$. The intial case of $n=1$ is a triviality since $c_{-} \neq c_{+}$. In the induction step, let $n>1$ and $0 \leq k \leq n$ such that $c_{\underline{\varepsilon}}=c(n, k)=c_{\eta}$. We may also assume $0<k<n$ without loss of generality because there is exactly one sequence in each of the cases $k=0$ and $k=n$. Note that if both $(\underline{\varepsilon}, \underline{\tilde{\eta}})$ and $(\underline{\eta}, \underline{\tilde{v}})$ have NC-pairings, then so does $(\underline{\varepsilon}, \underline{\tilde{v}})$ (resp., $(\underline{\eta}, \underline{\tilde{\varepsilon}}))$ which can easily be obtained by taking the product (resp., *) of the Temperley-Lieb diagrams associated to the NC-pairings. Consider $\underline{\varepsilon}(n, k) \in I^{n}$ given by $\varepsilon_{i}(n, k)=\left\{\begin{array}{ll}(-)^{i} & \text { if } 1 \leq i \leq k, \\ (-)^{i+1} & \text { if } k+1 \leq i \leq n\end{array}\right.$. Clearly, $c_{\underline{\varepsilon}(n, k)}=c(n, k)$; so, it is enough to show that $(\underline{\varepsilon}, \underline{\tilde{\varepsilon}}(n, k))$ has an NC-pairing. We begin by showing that there exists a $\underline{\nu} \in I^{n}$ such that $\nu_{1}=-=\varepsilon_{1}(n, k)$ and $(\underline{\varepsilon}, \underline{\tilde{v}})$ has an NC-pairing (and hence $c_{\underline{\varepsilon}}=c_{\underline{\nu}}$ by the 'if' part). Suppose first that $\varepsilon_{1}=+$. Since $0<k<n, \underline{\varepsilon}$ must have a pair of consecutive matching signs. Choose the left most consecutive matching pair in $\underline{\varepsilon}$ and change both the signs in the pair to obtain $\underline{\gamma} \in I^{n}$. Observe that there exists an NC-pairing for $(\underline{\varepsilon}, \tilde{\gamma})$. Also, the first consecutive matching pair in $\underline{\gamma}$ gets closer to the left side than the one in $\underline{\varepsilon}$. Apply this method succesively to obtain the desired $\underline{\nu}$. And, if $\varepsilon_{1}=-$, then simply take $\underline{\nu}=\underline{\varepsilon}$. Let $\underline{\nu^{\prime}}$ (resp., $\left.\underline{\varepsilon}^{\prime}(n, k)\right)$ be the sequence obtained from $\underline{\nu}$ (resp., $\left.\underline{\varepsilon}(n, k)\right)$ after removing the minus sign in the first entry. Since $c_{\underline{\nu^{\prime}}}=c_{\underline{\varepsilon^{\prime}}(n, k)}$, by induction assumption, there exists an NC-pairing for $\left(\underline{\varepsilon}^{\prime}(n, k), \underline{\nu}^{\prime}\right)$ and hence for $(\underline{\varepsilon}(n, k), \underline{\tilde{\tilde{\nu}}})$.

It is also true that for $\underline{\varepsilon}, \underline{\eta} \in I^{n}, c_{\underline{\varepsilon}}=c_{\underline{\eta}}$ if and only if $p_{\underline{\varepsilon}}$ and $p_{\underline{\eta}}$ are equivalent in $\tilde{P}_{n}$. Suppose $c_{\underline{\varepsilon}}=c_{\underline{\eta}}$. So, there exists an NC-pairing for $(\underline{\varepsilon}, \tilde{\underline{\eta}})$. Let $T$ be the Temperley-Lieb diagram associated to this NC-pairing. Since $p_{\underline{\varepsilon}}$ and $p_{\underline{\eta}}$ are minimal in $\tilde{P}_{n}$, therefore it is enough to check $p_{\underline{\varepsilon}}\left(\tilde{P}_{T}\right) p_{\underline{\eta}} \neq 0$ which easily follows by considering the scalar $\operatorname{tr}_{M_{n-1}}\left(p_{\underline{\varepsilon}}\left(\tilde{P}_{T}\right) p_{\underline{\eta}}\left(\tilde{P}_{T^{*}}\right) p_{\underline{\varepsilon}}\right)$ and then using the action of tangles to show it is nonzero (in fact, positive). In particular, $p(\underline{\varepsilon}, T, \underline{\tilde{\eta}}):=\sqrt{\frac{c_{\underline{\underline{\varepsilon}}}}{\operatorname{tr}_{M_{n-1}}\left(p_{\underline{\underline{\varepsilon}}}\left(\overline{\tilde{P}}_{T}\right) p_{\underline{\underline{q}}}\left(\tilde{P}_{T^{*}}\right)\right)}} p_{\underline{\varepsilon}}\left(\tilde{P}_{T}\right) p_{\underline{\eta}}$ is a partial isometry with $p_{\underline{\varepsilon}}$ (resp., $p_{\underline{\eta}}$ ) as its final (resp., initial) projection. To show that $p(\underline{\varepsilon}, T, \underline{\tilde{\eta}}$ ) is independent of $T$, consider two Temperley-Lieb elements $T_{1}$ and $T_{2}$ for two NC-pairings of $(\underline{\varepsilon}, \underline{\tilde{\eta}})$. It immediately follows from the action of tangles that $p\left(\underline{\varepsilon}, T_{1}, \underline{\tilde{\eta}}\right)\left(p\left(\underline{\varepsilon}, T_{2}, \underline{\tilde{\eta}}\right)\right)^{*} \in \mathbb{R}_{+}\left[p_{\underline{\varepsilon}}\left(\tilde{P}_{T_{1}}\right) p_{\underline{\eta}}\left(\tilde{P}_{T_{2}^{*}}\right) p_{\underline{\varepsilon}}\right]=\mathbb{R}_{+}\left[p_{\underline{\varepsilon}}\left(\tilde{P}_{T_{1} \cdot T_{2}^{*}}\right) p_{\underline{\varepsilon}}\right]=\mathbb{R}_{+} p_{\underline{\varepsilon}}$. Hence, $p\left(\underline{\varepsilon}, T_{1}, \underline{\tilde{\eta}}\right)=p\left(\underline{\varepsilon}, T_{2}, \underline{\tilde{\eta}}\right)$. So, we will denote the partial isometry simply by $p(\underline{\varepsilon}, \underline{\tilde{\eta}})$.

Consider the diagonal planar algebra $P$ in Proposition 6.5 and its perturbation $Q=Q^{\left(c_{-} c_{+}\right)^{-1}}=$ $P^{\left(z^{1 / 2}, z^{1 / 2}\right)}$ with respect to the weight $z:=\sqrt{\frac{c_{-}}{c_{+}}}(-,-)+\sqrt{\frac{c_{+}}{c_{-}}}(+,+)$. Define $\varphi: Q_{+} \rightarrow \tilde{P}_{+}$by

$$
Q_{+k}=P_{+k}=(\underline{\varepsilon}, \underline{\tilde{\eta}}) \stackrel{\varphi}{\mapsto} p(\underline{\varepsilon}, \underline{\tilde{\eta}}) \in \tilde{P}_{+k} .
$$

Clearly, $\varphi$ is a $*$-algebra isomorphism. By Proposition 2.3. it is enough to show that $\varphi$ is equivarent with respect to all tangles having positive colors on their discs and in fact, for the following tangles:

Right-inclusion tangles: For $\underline{\varepsilon}, \underline{\eta} \in I^{n}$ such that alt $t_{+}(\underline{\varepsilon}, \underline{\tilde{\eta}})=e$ (equivalently, $(\underline{\varepsilon}, \underline{\tilde{\eta}})$ has an NC-pairing), we have $\varphi \circ Q_{R I_{+n}}(\underline{\varepsilon}, \underline{\tilde{\eta}})=p(\underline{\varepsilon},-,-, \underline{\tilde{\eta}})+p(\underline{\varepsilon},+,+, \underline{\tilde{\eta}}) \in \tilde{P}_{+(n+1)}$. Thus, it is enough to show that $p(\underline{\varepsilon}, \underline{\tilde{q}})=$ $p(\underline{\varepsilon},-,-, \underline{\tilde{\eta}})+p(\underline{\varepsilon},+,+, \underline{\tilde{\eta}})$. Let $T$ be the Temperley-Lieb diagram corresponding to an NC-pairing for $(\underline{\varepsilon}, \underline{\tilde{\eta}})$; then, $R I_{+n} \circ T$ gives an NC-pairing for $(\underline{\varepsilon}, \pm, \pm, \underline{\tilde{\eta}})$. Further, we have $c_{(\underline{\varepsilon}, \pm)}=c_{\underline{\varepsilon}} c_{ \pm}^{(n+1)}$ and

$$
\operatorname{tr}_{M_{n}}\left(p_{(\underline{\varepsilon}, \pm)}\left(\tilde{P}_{R I_{+n} \circ T}\right) p_{(\underline{\eta}, \pm)}\left(\tilde{P}_{\left(R I_{+n} \circ T\right)^{*}}\right)\right)=\operatorname{tr}_{M_{n-1}}\left(p_{\underline{\varepsilon}}\left(\tilde{P}_{T}\right) p_{\underline{\eta}}\left(\tilde{P}_{T^{*}}\right)\right) c_{ \pm}^{(n+1)}
$$

which imply $p(\underline{\varepsilon}, \pm, \pm, \underline{\tilde{q}})=p(\underline{\varepsilon}, \underline{\tilde{\eta}}) p_{ \pm}^{(n+1)}$. Hence, the required equality holds.

Jones projection tangles: From the action of tangles on $\tilde{P}$, we have

$$
\begin{aligned}
\tilde{P}_{E_{+n}} & =\sum_{\underline{\varepsilon}^{\prime}, \underline{\varepsilon}^{\prime \prime} \in I^{n+1}} p_{\underline{\varepsilon}^{\prime}} \tilde{P}_{E_{+n}} p_{\underline{\varepsilon}^{\prime \prime}}=\sum_{\underline{\varepsilon} \in I^{n-1}, \eta, \nu \in I} p_{(\underline{\varepsilon}, \eta, \eta)} \tilde{P}_{E_{+n}} p_{(\underline{\varepsilon}, \nu, \nu)} \\
& =\sum_{\underline{\varepsilon} \in I^{n-1}, \eta, \nu \in I} \sqrt{\frac{\operatorname{tr}_{M_{n}}\left(p_{(\underline{\varepsilon}, \eta, \eta)}\left(\tilde{P}_{E_{+n}}\right) p_{(\underline{\varepsilon}, \nu, \nu)}\left(\tilde{P}_{E_{+n}}\right)\right)}{c_{(\underline{\varepsilon}, \eta, \eta)}}} p(\underline{\varepsilon}, \eta, \eta, \nu, \nu, \tilde{\tilde{\varepsilon}}) .
\end{aligned}
$$


Now, for $\underline{\varepsilon} \in I^{n-1}$ and $\eta, \nu \in I$, note that $\left(\tilde{P}_{E_{+n}}\right) p_{(\underline{\varepsilon}, \nu, \nu)}\left(\tilde{P}_{E_{+n}}\right)=\frac{c_{\nu}^{(n)}}{\sqrt{c_{-} c_{+}}} p_{\underline{\varepsilon}}\left(\tilde{P}_{E_{+n}}\right)$ and

$$
\begin{aligned}
\operatorname{tr}_{M_{n}}\left(p_{(\underline{\varepsilon}, \eta, \eta)} p_{\underline{\varepsilon}}\left(\tilde{P}_{E_{+n}}\right)\right) & =\sqrt{c_{-} c_{+}} \operatorname{tr}_{M_{n}}\left(\left(\tilde{P}_{E_{+n}}\right) p_{(\underline{\varepsilon}, \eta, \eta)}\left(\tilde{P}_{E_{+n}}\right)\right) \\
& =c_{\eta}^{(n)} \operatorname{tr}_{M_{n}}\left(p_{\underline{\varepsilon}}\left(\tilde{P}_{E_{+n}}\right)\right)=c_{\eta}^{(n)} \sqrt{c_{-} c_{+}} t_{M_{n-2}}\left(p_{\underline{\varepsilon}}\right)=c_{\eta}^{(n)} c_{\underline{\varepsilon}} \sqrt{c_{-} c_{+}}
\end{aligned}
$$

Since $c_{(\underline{\varepsilon}, \eta, \eta)}=c_{-} c_{+} c_{\underline{\varepsilon}}$, therefore $\tilde{P}_{E_{+n}}=\sum_{\underline{\varepsilon} \in I^{n-1}, \eta, \nu \in I} \sqrt{\frac{c_{\eta}^{(n)} c_{\nu}^{(n)}}{c_{-} c_{+}}} p(\underline{\varepsilon}, \eta, \eta, \nu, \nu, \underline{\tilde{\varepsilon}})$. On the other hand, using the weight $z$ of $P, Q_{E_{+n}}$ is equal to

$$
\left[\sum_{\underline{\varepsilon} \in I^{n-1}} \sqrt{\frac{c_{(-)^{n-1}-}}{c_{(-)^{n-1}+}}}(\underline{\varepsilon},-,-,-,-, \underline{\tilde{\varepsilon}})+(\underline{\varepsilon},-,-,+,+, \underline{\tilde{\varepsilon}})+(\underline{\varepsilon},+,+,-,-, \underline{\tilde{\varepsilon}})+\sqrt{\frac{c_{(-)^{n-1}+}}{c_{(-)^{n-1}}}}(\underline{\varepsilon},+,+,+,+, \underline{\tilde{\varepsilon}})\right] .
$$

Thus, $\varphi$ preserves the action of $E_{+n}$.

Left conditional expectation tangle: Fix $\nu, \nu^{\prime} \in I$ and $\underline{\varepsilon}, \underline{\eta} \in I^{n-1}$ such that alt $+\left(\nu, \underline{\varepsilon}, \underline{\tilde{\eta}}, \nu^{\prime}\right)=e$. Note that $Q_{E_{+n}^{\prime}}\left(\nu, \underline{\varepsilon}, \underline{\tilde{\eta}}, \nu^{\prime}\right)=\delta_{\nu=\nu^{\prime}} Q_{E_{+n}^{\prime}}(\nu, \underline{\varepsilon}, \underline{\tilde{\eta}}, \nu)=\delta_{\nu=\nu^{\prime}} \sqrt{\frac{c_{-\nu}}{c_{\nu}}} \sum_{\gamma \in I}(\gamma, \underline{\varepsilon}, \underline{\tilde{\eta}}, \gamma) \stackrel{\varphi}{\mapsto} \delta_{\nu=\nu^{\prime}} \sqrt{\frac{c_{-\nu}}{c_{\nu}}} \sum_{\gamma \in I} p(\gamma, \underline{\varepsilon}, \underline{\tilde{\eta}}, \gamma)$. On the other hand, $\tilde{P}_{E_{+n}^{\prime}}\left(p\left(\nu, \underline{\varepsilon}, \underline{\tilde{\eta}}, \nu^{\prime}\right)\right)=\tilde{P}_{E_{+n}^{\prime}}\left(p_{\nu} p\left(\nu, \underline{\varepsilon}, \underline{\tilde{\eta}}, \nu^{\prime}\right) p_{\nu^{\prime}}\right)=\delta_{\nu=\nu^{\prime}} \tilde{P}_{E_{+n}^{\prime}}(p(\nu, \underline{\varepsilon}, \underline{\tilde{\eta}}, \nu))$. Now, we may assume that $\operatorname{alt}_{+}(\nu, \underline{\varepsilon}, \underline{\tilde{\eta}}, \nu)=\bar{e}$ which implies alt $-(\underline{\varepsilon}, \underline{\tilde{\eta}})=e$ equivalently, $(\underline{\varepsilon}, \underline{\tilde{\eta}})$ has an NC-pairing given by a Temperley-Lieb diagram $T$ (say). Then, $T_{1}:=L I_{-(n-1)} \circ T$ induces an NC-pairing for $(\nu, \underline{\varepsilon}, \underline{\tilde{\eta}}, \nu)$. So, we have

$$
\begin{aligned}
\tilde{P}_{E_{+n}^{\prime}}(p(\nu, \underline{\varepsilon}, \underline{\tilde{\eta}}, \nu)) & =\sqrt{\frac{c_{(\nu, \underline{\varepsilon})}}{\operatorname{tr}_{M_{n-1}}\left(p_{(\nu, \underline{\varepsilon})}\left(\tilde{P}_{T_{1}}\right) p_{(\nu, \underline{\eta})}\left(\tilde{P}_{T_{1}^{*}}\right)\right)}} \tilde{P}_{E_{+n}^{\prime}}\left(p_{(\nu, \underline{\varepsilon})}\left(\tilde{P}_{T_{1}}\right) p_{(\nu, \underline{\eta})}\right) \\
& =\sqrt{\frac{c_{(\nu, \underline{\varepsilon})}}{\operatorname{tr}_{M_{n-1}}\left(p_{(\nu, \underline{\varepsilon})}\left(\tilde{P}_{T_{1}}\right) p_{(\nu, \underline{\eta})}\left(\tilde{P}_{T_{1}^{*}}\right)\right)}} \sqrt{\frac{c_{-\nu}}{c_{\nu}}} \sum_{\gamma \in I} p_{(\gamma, \underline{\varepsilon})}\left(\tilde{P}_{T_{1}}\right) p_{(\gamma, \underline{\eta})} .
\end{aligned}
$$

The last equality is obtained by using the action of tangles on $\tilde{P}$. To show $\varphi \circ Q_{E_{+n}^{\prime}}=\tilde{P}_{E_{+n}^{\prime}} \circ \varphi$, it remains to be shown that $\frac{c_{(\nu, \underline{\varepsilon})}}{\operatorname{tr}_{M_{n-1}}\left(p_{(\nu, \underline{\varepsilon})}\left(\tilde{P}_{T_{1}}\right) p_{(\nu, \underline{\eta})}\left(\tilde{P}_{T_{1}^{*}}\right)\right)}$ is independent of $\nu \in I$. Observe that the denominator

$$
\begin{aligned}
& \operatorname{tr}_{M_{n-1}}\left(p_{(\nu, \underline{\varepsilon})}\left(\tilde{P}_{T_{1}}\right) p_{(\nu, \underline{\eta})}\left(\tilde{P}_{T_{1}^{*}}\right)\right)=\left(c_{-} c_{+}\right)^{\frac{n}{2}} \tilde{P}_{T R_{+n}^{r}}\left(p_{(\nu, \underline{\varepsilon})}\left(\tilde{P}_{T_{1}}\right) p_{(\nu, \underline{\eta})}\left(\tilde{P}_{T_{1}^{*}}\right)\right) \\
= & \left(c_{-} c_{+}\right)^{\frac{n}{2}} \sqrt{\frac{c_{\nu}}{c_{-\nu}}} \sqrt{c_{-} c_{+}} \tilde{P}_{T R_{+n}^{r}}\left(p_{\underline{\varepsilon}}^{\prime}\left(\tilde{P}_{T_{1}}\right) p_{\underline{\eta}}^{\prime}\left(\tilde{P}_{T_{1}^{*}}\right)\right)=c_{\nu}\left(c_{-} c_{+}\right)^{\frac{n}{2}} \tilde{P}_{T R_{+n}^{r}}\left(p_{\underline{\varepsilon}}^{\prime}\left(\tilde{P}_{T_{1}}\right) p_{\underline{\eta}}^{\prime}\left(\tilde{P}_{T_{1}^{*}}\right)\right),
\end{aligned}
$$

where $\left.p_{\left(\omega_{1}, \cdots, \omega_{n-1}\right)}^{\prime}:=p_{\omega_{1}}^{(2)} p_{\omega_{2}}^{(3)} \cdots p_{\omega_{n-1}}^{(n)} \cdots, \omega_{n-1}\right) \in I^{n-1}$ and in the second equality, we replace the loop with $p_{\nu}$ in it by a loop with nothing on it, and the numerator $c_{(\nu, \underline{\varepsilon})}=c_{\nu} c_{-\underline{\varepsilon}}$. Hence, the required fraction becomes independent of $\nu \in I$.

\section{Questions}

Definition 7.1. A bimodule planar algebra is said to have trivial perturbation class if all its perturbation by positive weights, are spherical.

It will be interesting to find a set of necessary and sufficient conditions for a bimodule planar algebra having trivial perturbation class in terms of its principal graph(s); one can also consider this question in the more specific case of the Bisch-Haagerup planar algebras (see [BH], BDG1] and [BDG3]). Note that all finite depth or irreducible bimodule planar algebras have trivial perturbation class; so, for the Bisch Haagerup planar algebras, this question is relevant ony when the two subgroups have nontrivial intersection and the group generated by them is infinite.

Another interesting problem is to obtain a method of perturbing a bifinite bimodule to a new one whose associated planar algebra is the perturbation of the one associated to the bimodule which we start with. 
Note that, by Theorem 5.13, we may find a bifinite bimodule corresponding to a perturbation of the planar algebra associated to a given bifinite bimodule but we don't have any direct relation between these bimodules in terms of the weight. We will give answers to these questions in a forthcoming article.

Acknowledgements. The authors would like to thank Stefaan Vaes for his helpful comments, questions and views which helped us to be more precise at many instances. Thanks are also due to Steven Deprez, Vijay Kodiyalam and V. S. Sunder for several useful discussions. The authors would like to thank Vaughan Jones for suggesting Remark 6.6 to us during the NCGOA 2010 conference at Vanderbilt University.

\section{REFERENCES}

[Bis] D. Bisch, Bimodules, higher relative commutants and the fusion algebra associated to a subfactor, Operator algebras and their applications, 13-63, Fields Inst. Commun., 13, Amer. Math. Soc., Providence, RI, (1997).

[BDG1] D. Bisch, P. Das and S. K. Ghosh, The planar algebra of group-type subfactors, J of Func. Anal. 257(1), 20-46(2009).

[BDG2] D. Bisch, P. Das and S. K. Ghosh, The planar algebra of diagonal subfactors, Proc. Conference in honor of Alain Connes' 60th birthday, "Non-Commutative Geometry" April 2-6, 2007, IHP Paris, to appear, arXiv:0811.1084v2.

[BDG3] D. Bisch, P. Das and S. K. Ghosh, The planar algebra of group-type subfactors with cocycle, in preparation.

$[\mathrm{BH}] \quad$ D. Bisch and U. Haagerup, Composition of subfactors: new examples of infinite depth subfactors, Ann. Scient. Ec. Norm. Sup. 29, 329-383 (1996).

[Bur] M. Burns, Subfactors, planar algebras, and rotations, Ph.D. Thesis at the University of California Berkeley, 2003.

[EK] D. Evans and Y. Kawahigashi, Quantum symmetries on operator algebras, OUP New York (1998).

[Gho] S. K. Ghosh, Planar Algebras: A category theoretic point of view, J. Algebra, to appear, arXiv:math.QA/0810.4186.

[GHJ] F. Goodman, P. de la Harpe and V. F. R. Jones, Coxeter graphs and towers of algebras, Springer, Berlin, MSRI publication, (1989).

[GJS] A. Guionnet, V. F. R. Jones and D. Shlyakhtenko, Random matrices, free probability, planar algebras and subfactors, arXiv:0712.2904v2.

[Hia] Fumio Hiai, Minimizing indices of conditional expectations onto a subfactor, Publ. RIMS, Kyoto Univ., 24, 673-678 (1988).

[Jon1] V. F. R. Jones, Index for subfactors, Invent. Math., 72, 1-25 (1983).

[Jon2] V. F. R. Jones, Planar algebras, I, NZ J. Math., to appear, arXiv:math.QA/9909027

[Jon3] V. F. R. Jones, The planar algebra of a bipartite graph, Knots in Hellas'98 (Delphi), 94-117, (2000).

[Jon4] V. F. R. Jones, The annular structure of subfactors, L'Enseignement Math., 38, (2001).

[Jon5] V. F. R. Jones, Quadratic tangles in planar algebras, arXiv:1007.1158 1[math.OA].

[JP] V. F. R. Jones and D. Penneys, The embedding theorem for finite depth subfactor planar algebras, arXiv:1007. $3173 v 1[$ math.OA].

[JS] V. F. R. Jones and V. S. Sunder, Introduction to subfactors, LMS Lecture Notes Series, vol. 234, 162pp (1997).

[JSW] V. F. R. Jones, D. Shlyakhtenko and K. Walker, An orthogonal approach to the subfactor of a planar algebra, arXiv:0807.4146.

[Kas] C. Kassel, Quantum groups, Graduate Texts in Mathematics, 155, (1995).

[KS1] Vijay Kodiyalam and V. S. Sunder, On Jones' planar algebras, J. Knot Theory and its Ramifications, 13, No. 2, 219-247 (2004).

[KS2] Vijay Kodiyalam and V. S. Sunder, From subfactor planar aglebras to subfactors, Internat. Jour. Math., to appear, arXiv:0807.3704.

[PP1] M. Pimsner and S. Popa, Entropy and index for subfactors, Ann. Sci. Ec. Norm. Sup., 19, No. 1, 57-106 (1986).

[PP2] M. Pimsner and S. Popa, Iterating the basic construction, Trans. Amer. Math. Soc., 310, No. 1, 127-133 (1988).

[Pop1] Sorin Popa, Classification of subfactors: the reduction to commuting squares, Invent. Math., 101, No. 1, 19-43 (1990).

[Pop2] Sorin Popa, Sur la classification des sous-facteurs d'indice fini du facteur hyperfini, Comptes Rendus Acad. Sci. Paris, Ser. I, 311, 95- 100 (1990).

[Pop3] Sorin Popa, Classification of amenable subfactors of type II, Acta Math., 172, 163-255 (1994).

[Pop4] Sorin Popa, An axiomatization of the lattice of higher relative commutants, Invent. Math., 120, 427-445 (1995).

[Pop5] Sorin Popa, Universal construction of subfactors, J. Reine Angew. Math., 543, 39-81 (2002).

[Lei] Tom Leinster, Higher operads, higher categories, LMS Lecture Note Series, Vol. 298, 2008.

[Sun] V. S. Sunder, II -factors, their bimodules and hypergroups, Trans. Amer. Math. Soc., 330, 227-256 (1992).

Department of Mathematics, Katholieke Universiteit Leuven, Belgium

E-mail address: paramita.das@wis.kuleuven.be, shami.ghosh@wis.kulueven.be, ved.gupta@wis.kuleuven.be 